\title{
On the fine structure of the Cepheid metallicity gradient in the Galactic thin disk ${ }^{\star}, \star \star$
}

\author{
K. Genovali ${ }^{1}$, B. Lemasle ${ }^{2}$, G. Bono ${ }^{1,3}$, M. Romaniello ${ }^{4}$, M. Fabrizio ${ }^{5}$, I. Ferraro ${ }^{3}$, G. Iannicola ${ }^{3}$, C. D. Laney ${ }^{6,7}$, \\ M. Nonino ${ }^{8}$, M. Bergemann ${ }^{9,10}$, R. Buonanno ${ }^{1,5}$, P. François ${ }^{11,12}$, L. Inno ${ }^{1,4}$, R.-P. Kudritzki ${ }^{13,14,9}$, N. Matsunaga ${ }^{15}$, \\ S. Pedicelli ${ }^{1}$, F. Primas ${ }^{4}$, and F. Thévenin ${ }^{16}$
}

1 Dipartimento di Fisica, Università di Roma Tor Vergata, via della Ricerca Scientifica 1, 00133 Rome, Italy e-mail: katia.genovali@roma2.infn.it

2 Astronomical Institute Anton Pannekoek, Science Park 904, PO Box 94249, 1090 GE Amsterdam, The Netherlands

3 INAF - Osservatorio Astronomico di Roma, via Frascati 33, Monte Porzio Catone, Rome, Italy

${ }^{4}$ European Southern Observatory, Karl-Schwarzschild-Str. 2, 85748 Garching bei Munchen, Germany

5 INAF - Osservatorio Astronomico di Collurania, via M. Maggini, 64100 Teramo, Italy

6 Department of Physics and Astronomy, N283 ESC, Brigham Young University, Provo, UT 84601, USA

South African Astronomical Observatory, PO Box 9, Observatory 7935, South Africa

INAF - Osservatorio Astronomico di Trieste, via G.B. Tiepolo 11, 40131 Trieste, Italy

Max-Planck-Institut fur Astrophysik, Karl-Schwarzschild-Str. 1, 85741 Garching, Germany

10 Institute of Astronomy, University of Cambridge, Madingley Road, CB3 0HA, Cambridge, UK

11 GEPI - Observatoire de Paris, 64 avenue de l'Observatoire, 75014 Paris, France

12 UPJV - Université de Picardie Jules Verne, 80000 Amiens, France

${ }^{13}$ Institute for Astronomy, University of Hawai'i, 2680 Woodlawn Dr, Honolulu, HI 96822, USA

14 University Observatory Munich, Scheinerstr. 1, 81679 Munich, Germany

15 Kiso Observatory, Institute of Astronomy, School of Science, The University of Tokyo 10762-30, Mitake, Kiso-machi, Kiso-gun, 97-0101 3 Nagano, Japan

16 Laboratoire Lagrange, UMR 7293, Université de Nice Sophia-Antipolis, CNRS, Observatoire de la Côte d'Azur, 06300 Nice, France

Received 5 December 2013 / Accepted 19 March 2014

\section{ABSTRACT}

We present homogeneous and accurate iron abundances for 42 Galactic Cepheids based on high resolution $(R \sim 38000)$ high signal-to-noise ratio $(S / N \geq 100)$ optical spectra collected with UVES at VLT (128 spectra). The above abundances were complemented with high-quality iron abundances provided either by our group (86) or available in the literature. We were careful to derive a common metallicity scale and ended up with a sample of 450 Cepheids. We also estimated accurate individual distances for the entire sample by using homogeneous near-infrared photometry and the reddening free period-Wesenheit relations. The new metallicity gradient is linear over a broad range of Galactocentric distances $\left(R_{\mathrm{G}} \sim 5-19 \mathrm{kpc}\right)$ and agrees quite well with similar estimates available in the literature $(-0.060 \pm 0.002 \mathrm{dex} / \mathrm{kpc})$. We also uncover evidence that suggests that the residuals of the metallicity gradient are tightly correlated with candidate Cepheid groups (CGs). The candidate CGs have been identified as spatial overdensities of Cepheids located across the thin disk. They account for a significant fraction of the residual fluctuations, and also for the large intrinsic dispersion of the metallicity gradient. We performed a detailed comparison with metallicity gradients based on different tracers: OB stars and open clusters. We found very similar metallicity gradients for ages younger than $3 \mathrm{Gyr}$, while for older ages we found a shallower slope and an increase in the intrinsic spread. The above findings rely on homogeneous age, metallicity, and distance scales. Finally, by using a large sample of Galactic and Magellanic Cepheids for which accurate iron abundances are available, we found that the dependence of the luminosity amplitude on metallicity is vanishing.

Key words. stars: abundances - stars: variables: Cepheids - stars: oscillations - Galaxy: disk - open clusters and associations: general

\section{Introduction}

Recent findings concerning the metallicity gradient across the Galactic thin disk, based on high spectral resolution, high signal-to-noise $(\mathrm{S} / \mathrm{N})$ spectra, and on stellar tracers for which we can provide accurate individual Galactocentric distances, are

* Based on spectra collected with the spectrograph UVES available at the ESO Very Large Telescope (VLT), Cerro Paranal, (081.D-0928(A) PI: S. Pedicelli - 082.D-0901(A) PI: S. Pedicelli - 089.D-0767 PI: K. Genovali).

$\star \star$ Figure 1 and Tables 1, 3-7 are available in electronic form at http://www . aanda.org disclosing a new interesting scenario. The iron gradients traced by stellar populations younger than a few hundred Myr show a well-defined trend when moving from the inner to the outer disk regions. The iron abundances in the innermost disk regions $\left(R_{\mathrm{G}} \sim 5 \mathrm{kpc}\right)$ are well above solar $([\mathrm{Fe} / \mathrm{H}] \sim 0.4$, Andrievsky et al. 2002; Pedicelli et al. 2009; Luck et al. 2011; Luck \& Lambert 2011; Genovali et al. 2013, hereafter G13), while in the outer disk $\left(R_{\mathrm{G}} \sim 15 \mathrm{kpc}\right)$ they are significantly more metal-poor $([\mathrm{Fe} / \mathrm{H}] \sim-0.2 /-0.5$, Andrievsky et al. 2004; Yong et al. 2006; Lemasle et al. 2008). However, the young stellar populations in the two innermost Galactic regions showing ongoing star formation activity - the bar and the nuclear bulge - attain solar 
iron abundances, thus suggesting that the above regions have experienced different chemical enrichment histories (Bono et al. 2013).

The use of high-quality data and a homogeneous analysis of a large sample of classical Cepheids and young massive main-sequence stars provided the opportunity to overcome some of the systematics affecting early estimates of the metallicity gradient. However, current findings still rely on several assumptions that might introduce systematic errors.

i) Distances - Cepheids are very solid primary distance indicators, but they only trace young stellar populations. The use of red clump stars is very promising, since they are found throughout the innermost Galactic regions. However, their individual distances might be affected by larger uncertainties, since we are dealing with stellar populations covering a broad range of ages and metal abundances (Girardi \& Salaris 2001; Salaris \& Girardi 2002).

ii) Gradients - Recent spectroscopic investigations indicate that the use of homogeneous and accurate iron abundances decreases the spread along the radial gradient (G13). This means that they can be adopted to investigate the fine structure of the metallicity distribution (Lépine et al. 2011) and the possible occurrence of gaps and/or of changes in the slope (Lépine et al. 2013).

iii) Ages - The central helium burning phases of intermediate-mass stars $\left(M \sim 3.5-10 \quad M_{\odot}\right)$ take place along the so-called blue loops. During these phases an increase in stellar masses causes a steady increase in the mean luminosity. These are the reasons why classical Cepheids obey a period-age relation. However, the ages covered by Cepheids is quite limited ( 20-400 Myr). Most of the current chemical evolution models predict a steady decrease in slope of the metallicity gradient as a function of age (Portinari \& Chiosi 2000; Cescutti et al. 2007; Minchev et al. 2013). However, we still lack firm estimates of this effect since homogeneous estimates of distance, age, and chemical composition for a large sample of intermediate and old open clusters (Salaris et al. 2004; Carraro et al. 2007b; Yong et al. 2012) are still missing.

In this investigation we provide new accurate and homogeneous iron abundance estimates for 42 Galactic Cepheids based on high-resolution, high $\mathrm{S} / \mathrm{N}$ spectra acquired with UVES at VLT. The total sample includes estimates for 75 Cepheids ( 74 classical Cepheids and one Type II Cepheid - DD Vel - that will be discussed in a forthcoming paper), whose abundances were partially published in Genovali et al. (2013). We also analyzed three high spectral resolution spectra for the Cepheids TV CMa, RU Sct, X Sct - collected with NARVAL at the Télescope Bernard Lyot (TBL) ${ }^{1}$ that we adopted to double check current iron abundance estimates. We also added a new estimate of the FEROS spectrum for the Cepheid CE Pup whose metallicity was already available in the literature (Luck et al. 2011).

The above iron abundances were complemented with similar estimates provided either by our group (Lemasle et al. 2007, 2008; Romaniello et al. 2008, 53 Cepheids) or available in the literature (Yong et al. 2006; Luck et al. 2011; Luck \& Lambert 2011, 322 Cepheids). We ended up with a sample of 450 classical Cepheids, i.e., the $73 \%$ of the entire sample of known Galactic Cepheids according to the classical

\footnotetext{
1 Based on observations collected with TBL (USR5026) operated by OMP and INSU under program ID L072N06 (PI: B. Lemasle).
}

Cepheids list in the GCVS (candidate Cepheids are excluded from this estimate). For the entire sample, we estimated homogeneous distances based on reddening-free near-infrared period-Wesenheit (PW) relations (Inno et al. 2013).

This is the eighth paper of a series devoted to chemical composition of Galactic and Magellanic Cepheids (see the reference list). The name of the project is DIsk Optical and Near infrared Young Stellar Object Spectroscopy (DIONYSOS). The structure of the paper is the following. In Sect. 2 we present the spectroscopic data sets adopted in the current investigation and the method adopted to estimate the iron abundances. The photometric data and the individual distances are discussed in Sect. 3, together with a detailed analysis of the errors affecting Cepheid distances. Section 4 deals with the metallicity gradient, while in Sect. 5 we investigate the dependence of the metallicity gradient on stellar age. In this section the metallicity gradient is compared with the metallicity gradients based on younger tracers (OB stars) and with intermediate-age tracers (open clusters). In Sect. 6 we address in detail the fine structure of the metallicity gradient and perform a thorough analysis of the Cepheid radial distribution across the Galactic disk. Section 7 deals with the longstanding open problem concerning the dependence of the luminosity amplitude on the metallicity, while in Sect. 8 we summarize the results and briefly outline the future developments of this project.

\section{Spectroscopic data and iron abundances}

\subsection{Spectroscopic data}

In this investigation we present a spectral analysis based on high-resolution $(R \sim 38000)$ spectra collected with the UVES spectrograph available at the Nasmyth B focus of UT2/VLT Cerro Paranal telescope. Multi-epoch spectra for eleven Galactic Cepheids were collected during observing run B (P89). This sample includes 44 high-resolution spectra (from four to six spectra per star) for a total of eleven Cepheids. The covered spectral range is 4726-5804 $\AA$ and 5762-6835 $\AA$ over the two chips, collected by only using the red arm configuration and the cross disperser CD 3 (central wavelength at $580 \mathrm{~nm}$ ). The $\mathrm{S} / \mathrm{N}$ ranges from $\sim 50$ to $\sim 300$ (see Fig. 1). The seeing during the observations ranged from 0.5 arcsec to 2.5 arcsec, with a typical mean value of 1.2 arcsec, while the exposure times changed from $20 \mathrm{~s}$ to $1400 \mathrm{~s}$.

We make use of an additional UVES sample partially presented in G13. The spectra were collected at random pulsational phases between 2008 October and 2009 April using the DIC2 $(437+760)$ configuration with blue and red arms operating in parallel. The two arms cover the wavelength intervals 3750-5000 $\AA$ and 5650-7600/7660-9460 $\AA$ (two chips in the red arm). The exposure time ranges from $80 \mathrm{~s}$ to $2000 \mathrm{~s}$, while the seeing ranges from 0.6 arcsec to 2 arcsec with a mean value of 1.2 arcsec. The $\mathrm{S} / \mathrm{N}$ is typically better than $\sim 100$ for all the echelle orders. The complete spectroscopic sample includes 84 spectra for a total of 77 Cepheids. The spectra of three Cepheids - WW Mon, V641 Cen, GQ Ori - were analyzed, but they were not included in the abundance analysis because the $\mathrm{S} / \mathrm{N}$ of the individual spectra was not good enough. The entire sample of UVES spectra were reduced using the ESO UVES dedicated pipeline REFLEX v2.1 (Ballester et al. 2011).

For three Cepheids - TV CMa, RU Sct, X Sct - we also analyzed high $\mathrm{S} / \mathrm{N}$ spectra collected with NARVAL at TBL. NARVAL has a spectral resolution of $\sim 75000$ and covers the wavelength range 3700-10500 $\AA$. The NARVAL spectra were 
K. Genovali et al.: The fine structure of the metallicity gradient

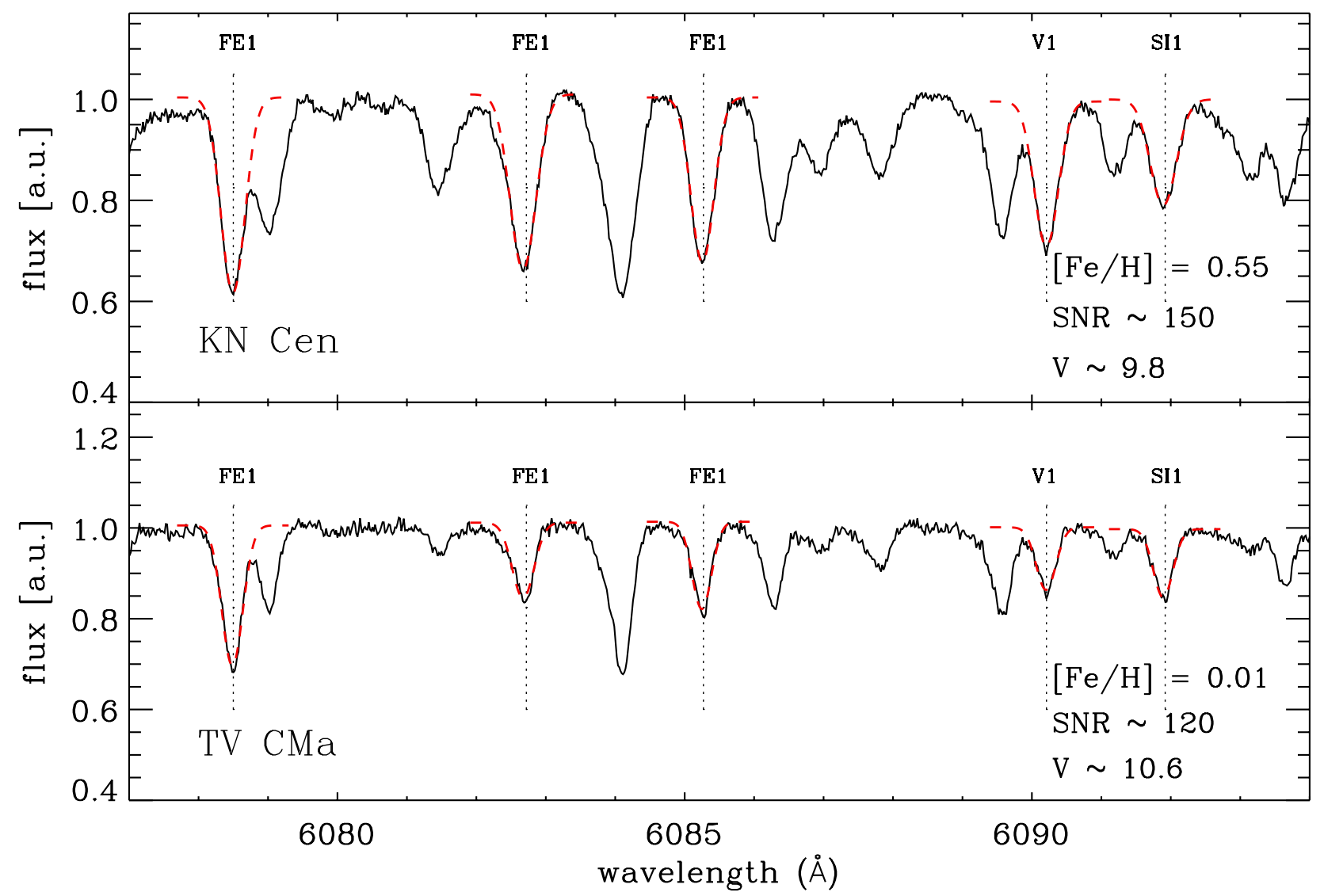

Fig. 2. High-resolution $(R \sim 38000)$ UVES spectrum of KN Cen $([\mathrm{Fe} / \mathrm{H}]=0.55 \pm 0.12$, Genovali et al. 2013) and TV CMa $([\mathrm{Fe} / \mathrm{H}]=0.01 \pm 0.07$, Genovali et al. 2013). The apparent visual magnitude and the $\mathrm{S} / \mathrm{N}$ in the spectral range $\lambda \sim 5650-7500 \AA$ (red arm) are also labeled. The vertical dashed lines display selected lines (Fe I 6078.50, Fe I 6082.72, Fe I 6085.27, V I 6090.21, Si I $6091.92 \AA$ ) adopted to estimate the individual $T_{\text {eff }}$ with the LDR method (Kovtyukh \& Gorlova 2000) (red dashed lines).

reduced using the data reduction software Libre-ESpRIT, written by Donati ${ }^{2}$. We also included the re-analysis of a FEROS ${ }^{3}$ spectrum for CE Pup (Luck et al. 2011) to better constrain possible systematic differences in the metallicity estimates of the outer disk.

As a whole, we provide in this investigation an updated spectroscopic estimate of the iron abundance for 42 classical Cepheids located either in the outer disk or in the solar neighborhood. Together with the iron abundances provided by G13 we ended up with a homogeneous metallicity sample of 74 classical Cepheids.

\subsection{Method}

We implemented a dedicated semi-automatic procedure able to determine the continuum and to fit the line profile by single or double Gaussian functions (see Fig. 2), depending on the line blending. We adopted the iron linelist presented in Genovali et al. (2013) and typically we measured the equivalent width (EW) of 100-200 Fe I and 20-40 Fe II lines, depending on the specific spectral range.

The abundance determination was performed by using the code calrai originally developed by Spite (1967) and continuously updated since then. Once the atmospheric

\footnotetext{
2 http://www.cfht.hawaii.edu/Instruments/Spectroscopy/ Espadons/Espadons_esprit.html

3 Pre-reduced spectra are available at http://archive.eso.org/ wdb/wdb/eso/repro/form
}

parameters are fixed, the code performs an interpolation over a grid of LTE, plane-parallel atmosphere models (MARCS, Gustafsson et al. 2008), and provides $[\mathrm{Fe} / \mathrm{H}]$ and its intrinsic error. The abundances of the other elements will be discussed in a forthcoming paper.

For each spectrum we computed the curves of growth for both neutral and ionized iron. The process is iterated until a good match between the predicted and observed equivalent widths (and thus the curves of growth) is obtained.

The effective temperature $-T_{\text {eff }}$ - for individual spectra was independently estimated by using the line depth ratio (LDR) method (Kovtyukh \& Gorlova 2000). Typically, we measured two dozen LDRs per spectrum (see, e.g., Fig. 2). The estimated $T_{\text {eff }}$ was validated by ensuring that Fe I does not depend on the excitation potential $\left(\chi_{\text {ex }}\right)$ i.e., the slope of Fe I vs. $\chi_{\text {ex }}$ is minimal. The surface gravity - $\log g$ - was derived by imposing the ionization balance between $\mathrm{Fe}_{\mathrm{I}}$ and $\mathrm{Fe}$ II. The micro-turbulent velocity $-v_{\mathrm{t}}-$ was estimated by minimizing the Fe I vs. EW slope. The atmospheric parameters estimated for each spectrum are given in Table 1.

The maximum EW value included in the analysis varies according to the metallicity itself and on the atmospheric parameters of the star. For a large fraction of our spectra we were able to use only relatively weak lines $(E W \leq 120 \mathrm{~m} \AA)$ located along the linear part of the curve of growth. In a few cases the spectra were lacking a significant number of weak lines (less than two $\mathrm{Fe}$ II lines); therefore, we also included in the analysis lines with EW up to $180 \mathrm{~m} \AA$. These lines cause a mild increase in the uncertainties affecting the correlated atmospheric 
Table 2. Impact of uncertainties affecting intrinsic parameters on iron abundance.

\begin{tabular}{|c|c|c|c|c|c|c|c|c|c|c|c|}
\hline \multirow[t]{2}{*}{ Name } & \multirow[t]{2}{*}{$T_{\text {eff }}$} & \multirow[t]{2}{*}[\mathrm{Fe}/\mathrm{H}]{} & \multirow[t]{2}{*}{$\log g$} & \multirow[t]{2}{*}{$\overline{v_{\mathrm{t}}}$} & \multirow[t]{2}{*}{ Element } & \multicolumn{2}{|c|}{$\overline{\Delta \Delta T_{\text {eff }}}$} & \multicolumn{2}{|c|}{$\Delta \log g$} & \multicolumn{2}{|c|}{$\overline{\Delta \Delta v_{\mathrm{t}}}$} \\
\hline & & & & & & $-100 \mathrm{~K}$ & $+100 \mathrm{~K}$ & -0.3 & +0.3 & -0.5 & +0.5 \\
\hline \multirow[t]{3}{*}{ V340 Ara } & 6060 & $0.22 \pm 0.01$ & 0.8 & 5.2 & Fe I & 0.17 & 0.31 & 0.28 & 0.22 & 0.27 & 0.23 \\
\hline & & & & & $\mathrm{Fe}$ II & 0.25 & 0.20 & 0.12 & 0.33 & 0.26 & 0.20 \\
\hline & & & & & {$[\mathrm{Fe} / \mathrm{H}]$} & 0.21 & 0.26 & 0.20 & 0.28 & 0.27 & 0.22 \\
\hline \multirow[t]{3}{*}{ V340 Ara } & 4820 & $0.23 \pm 0.18$ & 1.0 & 4.4 & $\mathrm{Fe}_{\mathrm{I}}$ & 0.14 & 0.30 & 0.20 & 0.21 & 0.27 & 0.15 \\
\hline & & & & & $\mathrm{Fe}_{\text {II }}$ & 0.38 & 0.22 & 0.14 & 0.46 & 0.42 & 0.20 \\
\hline & & & & & {$[\mathrm{Fe} / \mathrm{H}]$} & 0.26 & 0.26 & 0.17 & 0.34 & 0.35 & 0.18 \\
\hline \multirow[t]{3}{*}{ V500 Sco } & 5280 & $-0.03 \pm 0.12$ & 0.4 & 3.8 & $\mathrm{Fe} I$ & -0.11 & 0.06 & 0.01 & -0.07 & 0.04 & -0.07 \\
\hline & & & & & $\mathrm{Fe}$ II & 0.00 & -0.07 & -0.16 & 0.09 & 0.02 & -0.08 \\
\hline & & & & & {$[\mathrm{Fe} / \mathrm{H}]$} & -0.06 & -0.01 & -0.08 & 0.01 & 0.03 & -0.08 \\
\hline \multirow[t]{3}{*}{ V500 Sco } & 6020 & $-0.11 \pm 0.07$ & 1.1 & 4.0 & $\mathrm{Fe}_{\mathrm{I}}$ & -0.18 & -0.05 & -0.09 & -0.13 & -0.08 & -0.13 \\
\hline & & & & & $\mathrm{Fe}$ II & -0.11 & -0.11 & -0.21 & -0.01 & -0.05 & -0.15 \\
\hline & & & & & {$[\mathrm{Fe} / \mathrm{H}]$} & -0.15 & -0.08 & -0.15 & -0.07 & -0.07 & -0.14 \\
\hline \multirow[t]{3}{*}{ V510 Mon } & 5505 & $-0.16 \pm 0.06$ & 0.9 & 3.4 & $\mathrm{Fe} \mathrm{I}$ & -0.25 & -0.07 & -0.16 & -0.08 & -0.08 & -0.19 \\
\hline & & & & & $\mathrm{Fe}_{\text {II }}$ & -0.14 & -0.19 & -0.05 & -0.10 & -0.10 & -0.21 \\
\hline & & & & & {$[\mathrm{Fe} / \mathrm{H}]$} & -0.20 & -0.13 & -0.11 & -0.09 & -0.09 & -0.20 \\
\hline
\end{tabular}

Notes. Error budget for V340 Ara, V500 Sco, and V510 Mon. Columns 7 to 12 list plausible changes in atmospheric parameters: effective temperature $\left(T_{\text {eff }}\right)$, surface gravity $(\log g)$, and microturbulent velocity $\left(v_{\mathrm{t}}\right)$ together with their impact on Fe I and Fe II abundances. The mean $[\mathrm{Fe} / \mathrm{H}]$ abundance for each set of intrinsic parameters is also given.

parameters and become on the order of $\Delta \log g=0.3$ dex and $\Delta v_{\mathrm{t}}=0.5 \mathrm{~km} \mathrm{~s}^{-1}$. The impact that typical uncertainties on effective temperature, surface gravity, and microturbulent velocity have on the mean iron abundance are listed in Table 2. Data given in this table indicate that the difference in iron is on average smaller than 0.2 dex. Moreover, the difference in iron does not seem to depend, within the uncertainties, on the pulsation phase.

The mean iron abundances given in Col. 8 of Table 3 are the weighted mean of $[\mathrm{Fe} / / \mathrm{H}]$ and $[\mathrm{Fe} \mathrm{II} / \mathrm{H}]$ with associated errors, i.e., $\sigma_{\mathrm{Fe}}=\sqrt{\sigma_{\mathrm{Fe} I}^{2}+\sigma_{\mathrm{Fe} \mathrm{II}}^{2}}$, where $\sigma_{\mathrm{Fe} \text { I }}$ and $\sigma_{\mathrm{Fe} I I}$ are the standard deviations of $[\mathrm{Fe} \mathrm{I} / \mathrm{H}]$ and $[\mathrm{Fe} \mathrm{I} / \mathrm{H}]$ estimates given by the lines measured in a single spectrum. For Cepheids in our sample with multiple measurements the weighted average abundance and the standard deviation $\sigma_{\mathrm{Fe}}=\sqrt{\sum_{i} \sigma_{\mathrm{Fe}, \mathrm{i}}^{2}}$ are also listed. The iron abundances were estimated by assuming the solar iron abundance provided by Grevesse et al. (1996), i.e., $A(\mathrm{Fe})_{\odot}=7.50$.

In order to validate current iron abundances we adopted the NARVAL spectra, since they have a spectral resolution that is a factor of two higher than the UVES spectra and similar S/Ns. We found that the iron estimates for X Sct, TV CMa and RU Sct based on the NARVAL spectra agree quite well with those based on UVES spectra, and the difference is on average smaller than $\sim 0.1$ dex.

\section{Photometric data and distance estimates}

\subsection{Photometric data}

In order to provide a homogeneous sample of Galactocentric distances $\left(R_{\mathrm{G}}\right)$, we adopted near-infrared (NIR) photometric data together with the reddening-free PW relations in the $J, H, K_{\mathrm{s}}$ bands derived by Inno et al. (2013). We estimated individual distances for a significant fraction of Galactic Cepheids (93\% of the known Galactic Cepheids). To improve the precision of individual Cepheid distances, we adopted the NIR photometric catalogs provided by Laney \& Stobie (1992) and by Monson \& Pierce (2011). The above subsamples were complemented with NIR photometry from the 2MASS catalog.

The SAAO data set includes published mean magnitudes from Laney \& Stobie (1992) and new multi-epoch measurements (C.D. Laney, priv. comm.). The individual NIR measurements of the former sample cover the entire pulsation cycle and the accuracy of mean $J, H, K_{\mathrm{s}}$ magnitudes is typically better than $0.01 \mathrm{mag}$. Some of the Cepheids in the latter sample lack detailed coverage of the light curve. For these objects the number of phase points ranges from four to 14 and they are marked with a dagger in the column notes of Table 3. The mean magnitudes were estimated using a cubic spline. The SAAO NIR magnitudes were transformed into the 2MASS photometric system by using the transformations provided by Koen et al. (2007).

We also adopted the NIR photometric catalog from Monson \& Pierce (2011). They provided accurate NIR magnitudes for 131 northern hemisphere Cepheids. Their NIR mean magnitudes were transformed into the 2MASS photometric system using the calibrating equations provided by the same authors. The measurements properly cover the entire pulsation cycle and the typical accuracy on the mean magnitudes is better than $0.01 \mathrm{mag}$.

The above samples were complemented with 2MASS single-epoch NIR observations (Skrutskie et al. 2006). The mean magnitude for Fundamental (FU) Cepheids was estimated by using single-epoch photometry and the light-curve template provided by Soszyński et al. (2005). The pulsation properties required to apply the template (epoch of maximum, optical amplitudes, periods) come from the General Catalog of Variable Stars $^{4}$ (GCVS; Samus et al. 2009), with the exception of a few objects for which we adopted the pulsation periods provided by Luck \& Lambert (2011). The error on the mean NIR magnitudes was estimated as $\sigma_{J, H, K_{\mathrm{s}}}^{2}=\sigma_{\text {phot }}^{2}+\sigma_{\text {temp }}^{2}$, where $\sigma_{\text {phot }}$ is the intrinsic photometric error - typically of the order of 0.03 mag for the Cepheids in the 2MASS sample - and $\sigma_{\text {temp }}=0.03$ mag

4 http://www.sai.msu.su/gcvs/gcvs/index.htm 
is the uncertainty associated with the intrinsic scatter of the template.

We did not estimate the NIR mean magnitudes of classical Cepheids pulsating either in the first overtone (FO) or as mixed-mode pulsators (CEP(B)). Their mean magnitudes are the original single-epoch 2MASS measurements, because we still lack either the light-curve template for FOs or the epoch of maximum. This subsample is marked with an asterisk in the last column of Table 3 .

In order to provide an estimate of the uncertainty affecting distance estimates based on NIR single-epoch data (FU and FOs), we associated to this photometric sample a cautionary total error of $\sigma_{J, H, K_{\mathrm{s}}}=\sqrt{\sigma_{\text {phot }}^{2}+\left(A_{J, H, K_{\mathrm{s}}} / 2\right)^{2}}$, where $\left(A_{J, H, K_{\mathrm{s}}} / 2\right)$ is the semi-amplitude in the specific band. The NIR amplitudes were estimated by using empirical relations for the ratio between optical and NIR amplitudes. In particular, we adopted the $A_{\mathrm{NIR}} / A_{I}$ ratios provided by Soszyński et al. (2005) for FU Cepheids with $\log P \leq 1.3: A_{J} / A_{I}=0.63, A_{H} / A_{I}=0.50$, $A_{K} / A_{I}=0.49 \mathrm{mag}$. We estimated the amplitude in the $I$-band $A_{I}$ by using the optical ratios $A_{I}=0.62 A_{V}$ and $A_{I}=0.42 A_{B}$ according to the intrinsic parameters available in the GCVS.

For the FO pulsators we adopted the ratio between optical and NIR amplitudes for FU Cepheids with $\log P \leq 1.2$ (see Klagyivik \& Szabados 2009). This assumption relies on the theoretical and empirical evidence that FOs, once their period is fundamentalized, display pulsation properties very similar to FU Cepheids with periods shorter than $\log P \leq 1.2$.

We compared the above estimates with a dozen complete NIR FO light-curves available in the Laney sample and we found that in every case the observed ratios are quite similar to or lesser than the estimated ones. For double-mode and putative classical Cepheids we used instead the relations $A_{\mathrm{NIR}} / A_{V}$ provided by Soszyński et al. (2005) for classical Cepheids.

For the objects in common in more than one sample (SAAO, Monson \& Pierce 2011, 2MASS) we adopted the most accurate mean magnitude values.

\subsection{Distance determination}

The individual distance moduli were estimated as the weighted mean of the three different distance moduli obtained by adopting the NIR $(H, J-H ; K, J-K ; K, H-K)$ PW relations provided by Inno et al. (2013). The individual distance moduli are listed in Col. 9 of Tables 3 and 4 with their uncertainties. The Galactocentric distances listed in Col. 10 of Tables 3 and 4 were estimated assuming a solar Galactocentric distance of $7.94 \pm 0.37 \pm 0.26 \mathrm{kpc}$ (Groenewegen et al. 2008; Matsunaga et al. 2013, and references therein). The final error on $R_{\mathrm{G}}$ accounts for errors affecting both the solar Galactocentric and heliocentric distances.

We tested that differences among individual distances based on single-epoch 2MASS photometry with those based either on SAAO or on Monson \& Pierce (2011) NIR photometry are marginal $(3 \%$ on average with a standard deviation of $7 \%$ ). We also compared current individual distances based on NIR PW relations with individual distances estimated using two different flavors of the IRSB method and we found that the mean difference over the entire sample ranges from $8 \pm 2 \%$ (Groenewegen 2013, 130 stars in common) to $4 \pm 2 \%$ (Storm et al. 2011a, $\sim 80$ stars in common). The mean difference between our distances and the distances from Luck \& Lambert (2011) based on optical period-luminosity relations is of the order of $3 \pm 1 \%$ ( 400 stars in common). On the other hand, the typical dispersion between current and literature distances ranges from $17 \%$ (our-Luck) to $22 \%$ (our-Groenewegen). This suggests that the use of homogeneous NIR photometry and solid distance diagnostics have a major impact on the decrease of the intrinsic dispersion of Galactocentric distances.

\section{The metallicity gradient}

\subsection{Spectroscopic data sets}

We compared our new homogeneous estimates (current sample plus stars in Genovali et al. 2013, G13) with the iron abundances provided either by our group (Lemasle et al. 2007, 2008, LEM; Romaniello et al. 2008, ROM; Pedicelli et al. 2010, PED) or in the literature (Luck et al. 2011, LII; Luck \& Lambert 2011, LIII; Sziládi et al. 2007, SZI; Yong et al. 2006, YON).

The increase in the number of Cepheids in common among the different data sets allowed us to better evaluate the possible occurrence of a systematic difference in the metallicity distribution. The difference in iron abundance among the different samples are the following:

$\Delta[\mathrm{Fe} / \mathrm{H}](\mathrm{LIII}-\mathrm{ROM})=0.11 \pm 0.11(22)$,

$\Delta[\mathrm{Fe} / \mathrm{H}](\mathrm{LII}-\mathrm{LEM})=0.08 \pm 0.12(51)$,

$\Delta[\mathrm{Fe} / \mathrm{H}](\mathrm{LIII}-\mathrm{YON})=0.34 \pm 0.20(20)$,

$\Delta[\mathrm{Fe} / \mathrm{H}](\mathrm{LII}-\mathrm{G} 13)=-0.05 \pm 0.11(45)$,

$\Delta[\mathrm{Fe} / \mathrm{H}](\mathrm{LIII}-\mathrm{G} 13)=0.03 \pm 0.08(33)$.

The numbers in parentheses give the number of objects in common among the different data sets. The difference with the double-mode Cepheids by Sziládi et al. (2007) was not estimated, since we only have one object in common.

The typical difference is on average smaller than 0.1 dex. Our results for CE Pup and HW Pup further support the systematic difference between iron abundances provided by Yong et al. (2006) and similar estimates available in the literature (Lemasle et al. 2008; Luck et al. 2011; Luck \& Lambert 2011). In order to provide a homogeneous metallicity scale for a large sample of Galactic Cepheids, we applied the above differences to the quoted data sets. Column 7 in Table 4 lists the original iron abundances, while Col. 8 gives the rescaled iron abundance.

\subsection{The iron abundance gradient}

In this investigation we analyze the metallicity gradient using 63 homogeneous metallicity estimates based on single-epoch UVES spectra. Among them 33 were already presented in Genovali et al. (2013). We also include in the analysis new weighted mean abundances for eleven Cepheids observed from four to six times with UVES at random pulsational phases (V340 Ara, AV Sgr, VY Sgr, UZ Sct, Z Sct, V367 Sct, WZ Sgr, XX Sgr, KQ Sco, RY Sco, V500 Sco), collected either in observing run A (P81-P82, with the exception of V500 Sco) or in observing run B (P89, see Table 1). We confirm the previous findings by Andrievsky et al. (2005) and references therein that the iron abundance estimates, within the errors, are not phase-dependent. Moreover, we provide an independent estimate of the FEROS spectrum of the outer disk Cepheid CE Pup whose iron abundance was originally determined by Luck et al. (2011). A more detailed analysis of the multi-epoch spectra will be presented in a forthcoming paper.

The top panel of Fig. 4 shows the iron abundances based on UVES multi-epoch spectra of the observing run B (11 dark red circles), on UVES single-epoch spectra of the observing run A 

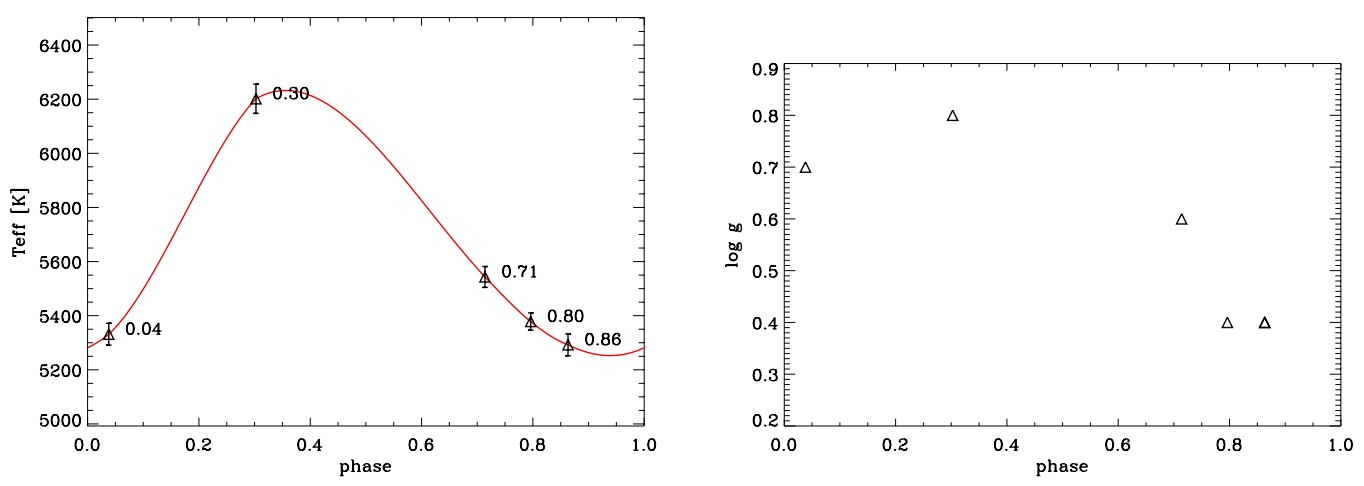

Fig. 3. Top: effective temperature curve vs. pulsational phase for the Cepheid RY Sco as derived using the LDRs method (see text for details). The adopted spectra are the same as in Fig. 1 . The $T_{\text {eff }}$ estimates derived for each spectrum and its error are plotted as black triangles. The red line shows the spline fit. The numbers plotted on top of the individual measurements show the pulsation phase. Bottom: same as the top, for the surface gravity.

(30 red circles), and on the FEROS spectrum (light blue circles) as a function of the Galactocentric distances $\left(R_{\mathrm{G}}\right)$. The blue circles display the iron abundances provided by Genovali et al. (2013) based on UVES single-epoch spectra of the observing run A (33 stars). Together with the current sample, we also included iron abundances for Galactic Cepheids estimated by our group using the same approach and similar data (Lemasle et al. 2007, 2008; 39 objects, green circles; Romaniello et al. 2008; 14 objects, yellow circles). The current data set covers a range in Galactocentric distances of more than $10 \mathrm{kpc}(4 \lesssim$ $R_{\mathrm{G}} 15 \mathrm{kpc}$ ). We estimated the metallicity gradient (dashed line) and we found $[\mathrm{Fe} / \mathrm{H}]=0.49 \pm 0.03-0.051 \pm 0.003 R_{\mathrm{G}} / \mathrm{kpc}$. The new slope and zero-point agree quite well with similar estimates available in the literature (Luck \& Lambert 2011; Lemasle et al. 2013). The spread in iron appears to be homogeneous over the entire Galactocentric range, but in the innermost disk regions it increases and becomes of the order of $0.5 \mathrm{dex}$. We also included Cepheid iron abundances available in the literature: Yong et al. (2006); Sziládi et al. (2007); Luck \& Lambert (2011); Luck et al. (2011) (322 objects, black circles). The priority for objects in common among different data sets was given to the current sample, then to iron abundances obtained by our group, and finally to abundances available in the literature.

It is worth mentioning that we have been able, thanks to the current large and homogeneous data set of NIR mean magnitudes, to include in the analysis of the metallicity gradient 18 Cepheids for which the iron abundance was provided by Luck \& Lambert (2011), but for which the individual distances were not available. We ended up with a sample of 450 Cepheids with a homogeneous metallicity scale and a homogeneous distance scale.

The metallicity gradient we found is $[\mathrm{Fe} / \mathrm{H}]=0.57 \pm 0.02$ $-0.060 \pm 0.002 R_{\mathrm{G}} / \mathrm{kpc}$, in very good agreement with previous results from Luck \& Lambert (2011) based on a similar number of Cepheids. We note that to identify possible outliers, we performed a preliminary gradient estimate and we found $[\mathrm{Fe} / \mathrm{H}]=0.54 \pm 0.02-0.057 \pm 0.002 R_{\mathrm{G}} / \mathrm{kpc}$. Subsequently, we neglected four Cepheids - BC Aql, HK Cas, EK Del, GP Per - with a gradient residual greater than $3 \sigma$. Three out of the four neglected Cepheids are classified in the GCVS as candidate Cepheids (CEP), while HK Cas is very high on the Galactic plane and it has been classified as an Anomalous Cepheid by Luck \& Lambert (2011).

The new metallicity gradient still shows a large intrinsic dispersion around the solar circle and in the outer disk (Fig. 4) with the possible occurrence either of a change in the slope or of a shoulder for $7 \leq R_{\mathrm{G}} \leq 10 \mathrm{kpc}$, as suggested by Twarog et al. (1997); Caputo et al. (2001), and Andrievsky et al. (2004).

To further constrain the nature of the spread in iron along the metallicity gradient the referee suggested that we check its dependency on the distance from the Galactic plane. We selected the Cepheids in our sample with a distance above the Galactic plane smaller than $300 \mathrm{pc}$ and we found that the gradient is quite similar: $[\mathrm{Fe} / \mathrm{H}]=0.49 \pm 0.03-0.052 \pm 0.004 R_{\mathrm{G}} / \mathrm{kpc}$. We performed the same test by using the entire sample and the gradient is once again minimally affected, we found $[\mathrm{Fe} / \mathrm{H}]=$ $0.53 \pm 0.02-0.055 \pm 0.002 R_{\mathrm{G}} / \mathrm{kpc}$. The subsample located closer to the Galactic plane was also adopted to constrain the spread in iron of the outer disk $\left(R_{\mathrm{G}} \geq 13 \mathrm{kpc}\right)$. We found that the spread decreases from 0.17 dex ( 30 Cepheids) to 0.13 dex (9 Cepheids). This means that the difference decreases from $13 \%$ to $5 \%$ higher than the mean spread over the entire disk ( $0.11 \mathrm{dex})$.

It was also noted that the spread in iron abundance around the solar circle is larger than the spread in the region between 10 and $14 \mathrm{kpc}$ and it was suggested that the difference might be caused by a different azimuthal distributions of the Cepheids in the two disk regions. To further constrain the dependence of the spread on the azimuthal distribution we estimated, following Genovali et al. (2013), the metallicity distribution of the four Galactic quadrants. We found that the $\sigma$ of the four distributions attain very similar values $(0.013 \pm 0.01 \mathrm{dex})$, while the mean iron abundance increases by almost 0.2 dex when moving from quadrants I/II to quadrants III/IV (see Fig. 3 in Genovali et al. 2013). The reader interested in a more quantitative analysis of the variation of the spread along the metallicity gradient is referred to Sect. 6.

The iron abundances of the current investigation cover the outer disk $R_{\mathrm{G}} \geq 13 \mathrm{kpc}$ and together with iron abundances provided either by our group or available in the literature they provide a detailed sampling over a broad range of Galactocentric distances ( $4 \leq R_{\mathrm{G}} \leq 19 \mathrm{kpc}$ ). Data plotted in Fig. 4 display a steady increase in the metallicity dispersion when moving from the solar circle to the outer disk. It is interesting to note that Cepheids located in the outer disk also show larger distances from the Galactic plane $(|z| \geq 400 \mathrm{pc}$ ) when compared with inner disk and solar circle Cepheids (see Fig. 6). However, we did not find a clear correlation between distance from the Galactic plane and metallicity. To constrain the above difference on a more quantitative basis, we analyzed the Cepheid azimuthal distribution and we found, as expected (Kraft \& Schmidt 1963), that they are on average $\sim 38 \pm 13$ pc below the Galactic plane and their $\sigma$ is $\sim 270 \mathrm{pc}$. However, the fraction of Cepheids 

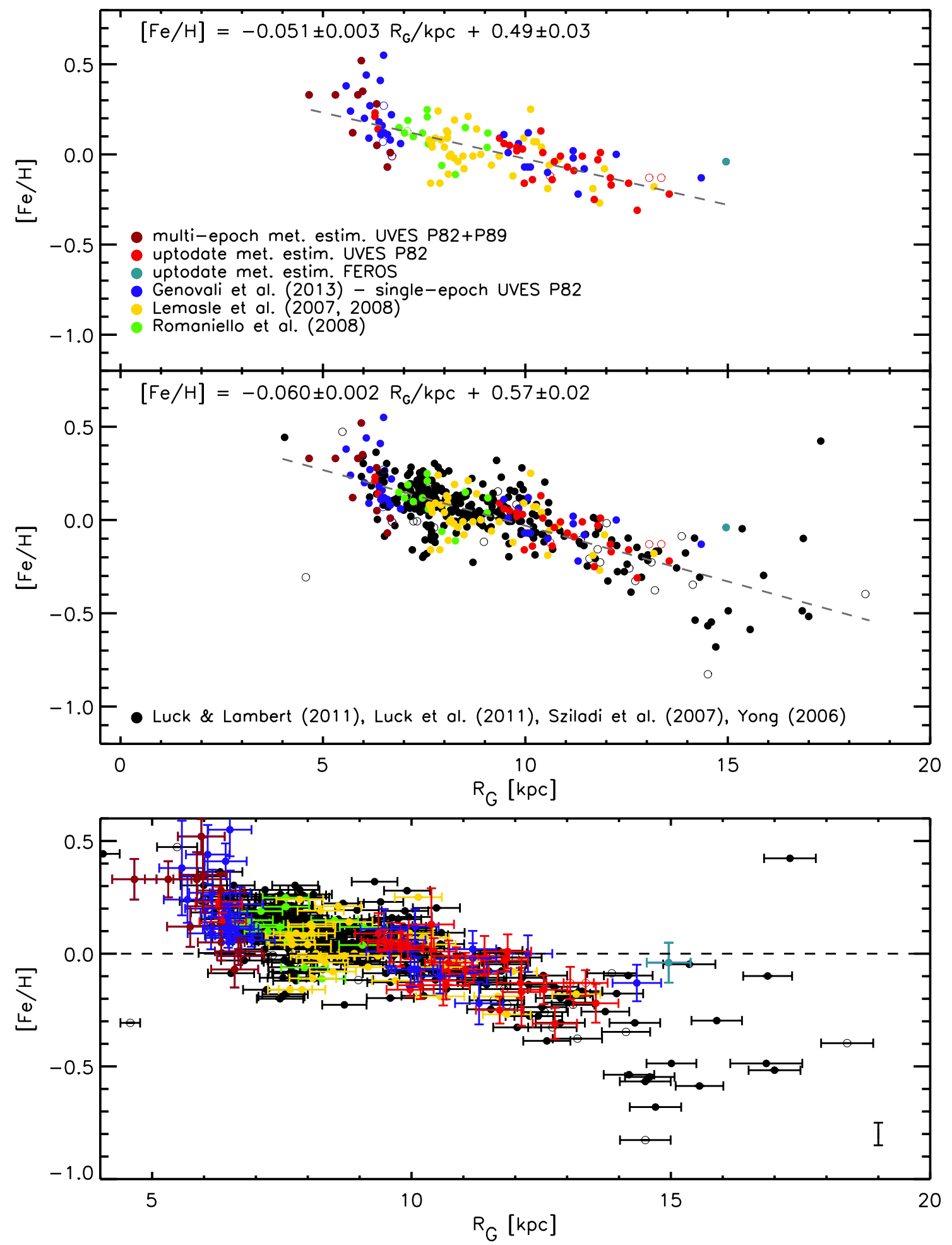

Fig. 4. Top: iron abundances of Galactic classical Cepheids vs. Galactocentric distance. Spectroscopic measurements based on different data sets are plotted with different colors. They include updated iron abundances based on current UVES spectra (30, red) and on multi-epoch UVES spectra (11, dark red); as well as updated iron abundance for CE Pup based on a FEROS spectrum (light blue) and iron abundances provided by our group: Genovali et al. (2013) (33, blue), Lemasle et al. (2007), Lemasle et al. (2008) (39, yellow), Romaniello et al. (2008) (14, green). Cepheids that according to the General Catalog of Variable Stars (Samus et al. 2009) are candidate classical Cepheids were plotted with open circles. The gray dashed line shows the metallicity gradient. Middle: same as the top, but the iron abundances include our measurements and those available in the literature: Luck et al. (2011), Luck \& Lambert (2011), and Sziládi et al. (2007) (322, black). The gray dashed line shows the metallicity gradient based on the entire sample. Bottom: zoom of the top panel for Galactocentric distances ranging from $4 \mathrm{kpc}$ to $20 \mathrm{kpc}$. The bars on individual Cepheids display the uncertainty both on iron abundance and on distance. The vertical black bar in the bottom right corner shows the mean uncertainty on Luck \& Lambert (2011) and Romaniello et al. (2008) iron abundances. 


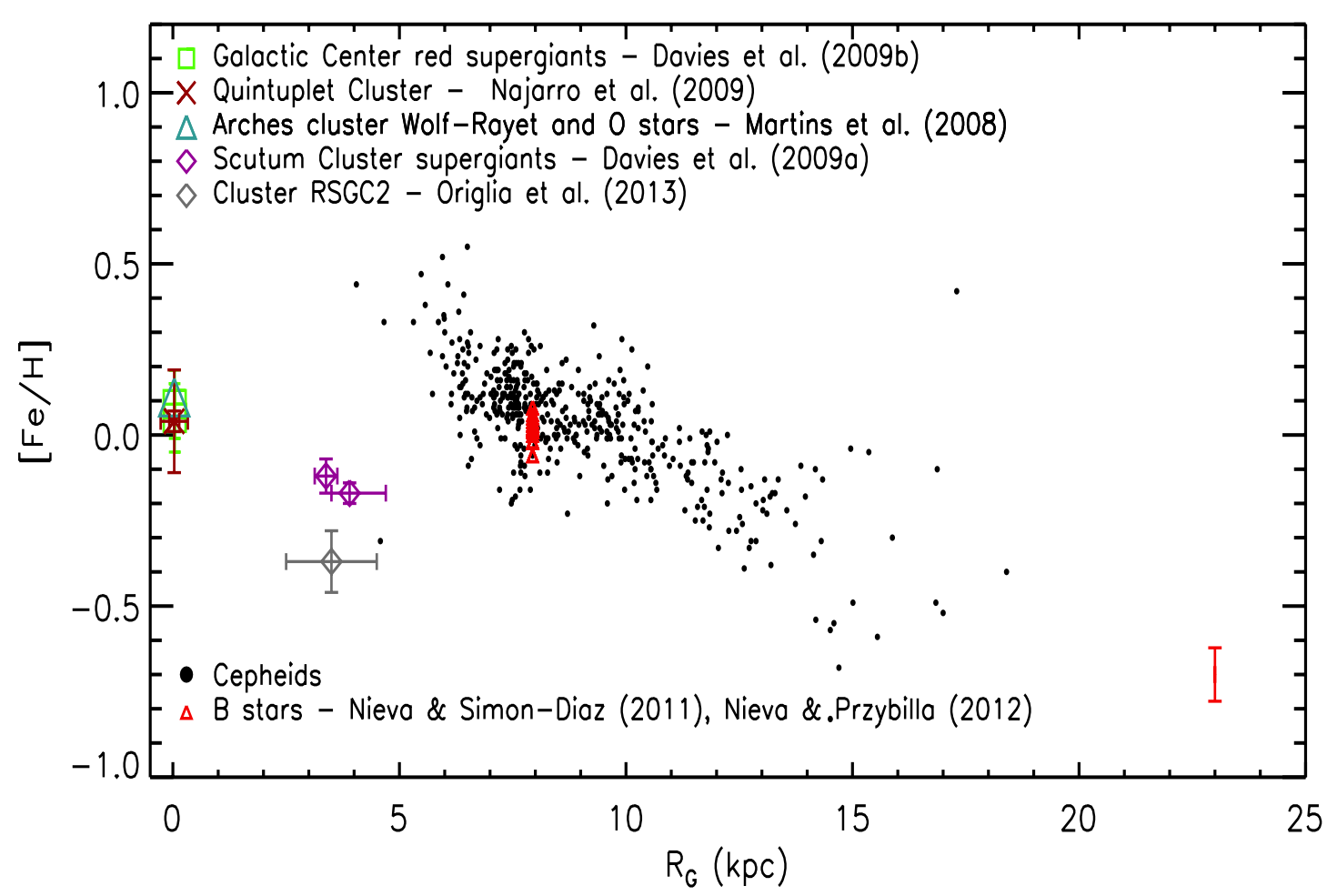

Fig. 5. Comparison of the Cepheid (black dots) metallicity gradient with iron abundances of early B-type stars located either in the solar neighborhood or in the Orion star forming region (Nieva \& Simón-Díaz 2011; Nieva \& Przybilla 2012). The green square marks the iron abundance of the two red supergiants in the Galactic center measured by Davies et al. (2009a), the magenta diamonds the 26 red supergiants in the Scutum Clusters measured by Davies et al. (2009b), the gray diamond the three red supergiants in the cluster RSGC2 measured by Origlia et al. (2013), the red cross the two luminous blue variables (LBVs) in the Quintuplet cluster (Najarro et al. 2009), and the light-blue triangle three Wolf-Rayet and two O-type stars in the Arches cluster (Martins et al. 2008). The red vertical bar in the bottom right corner shows the mean uncertainty on iron abundances.

located at distances from the Galactic plane larger than $1 \sigma$ increases from $4 \%$ for $R_{\mathrm{G}}$ smaller than $10 \mathrm{kpc}$ to $38 \%$ at larger Galactocentric distances.

It is worth mentioning that the evidence of an increase in the dispersion of the iron abundance in the outer disk is also supported by the fact that the use of homogeneous iron abundances has further decreased the intrinsic spread from 0.12 (see Fig. 2 of Genovali et al. 2013) to 0.10 dex (see Fig. 4) for $R_{\mathrm{G}} \sim 11-15 \mathrm{kpc}$.

\section{Comparison between Cepheid and independent metallicity gradients}

\subsection{Young tracers}

During the last few years several investigations have addressed the open problem concerning the age dependence of the metallicity gradient. This issue has been investigated not only from the empirical point of view (see, e.g., Maciel et al. 2003; Nordström et al. 2004; Henry et al. 2010; Yong et al. 2012) but also from the theoretical. In particular, there have been discussions regarding the role that different stellar tracers can play in constraining the chemical tagging not only in spatial distribution but also in time (Freeman \& Bland-Hawthorn 2002).

To further constrain the age effect on the metallicity gradient we collected several abundance gradients based on different stellar tracers.

Figure 5 shows the comparison between the metallicity gradient based on Cepheids with the iron abundance of almost three dozen of early B-type stars (red triangles) located either in the solar neighborhood (Nieva \& Przybilla 2012) or in the nearby Orion star forming region (Nieva \& Simón-Díaz 2011). The crucial advantage of this set of measurements is that they are based on high-resolution, high $\mathrm{S} / \mathrm{N}$ spectra; they are homogenous; and they also account for non-LTE effects (Przybilla et al. 2011).

The comparison of the iron abundances further supports the evidence that early B-type stars and classical Cepheids display similar abundance in the solar neighborhood. The spread in iron of the B-type stars is narrower (see the red vertical error bar plotted in the right corner) than the Cepheids, but they also cover a narrow disk region. We note that the comparison appears even more compelling if we consider that B-type stars are the typical progenitors of classical Cepheids.

The above scenario concerning the iron abundance gradient of young stellar tracers shows a stark difference when compared with iron abundances of young stars (red supergiants, luminous blue variables, Wolf-Rayet and O-type stars) located either in the nuclear bulge or in the near end of the Galactic bar (Martins et al. 2008; Davies et al. 2009a,b; Najarro et al. 2009). The above spectroscopic measurements suggest either a solar or a subsolar iron abundance. This finding was reliably confirmed by Origlia et al. (2013) by using high-spectral resolution $(R \sim 50000)$ NIR spectra collected with GIANO at TNG. They found that the mean iron abundance of three RSGs located in the RSGC2 cluster are subsolar. This finding does not seem to be supported by recent chemical evolution models by Minchev et al. (2013), since they predict in the innermost Galactic regions present-day super-solar iron abundances. 
K. Genovali et al.: The fine structure of the metallicity gradient

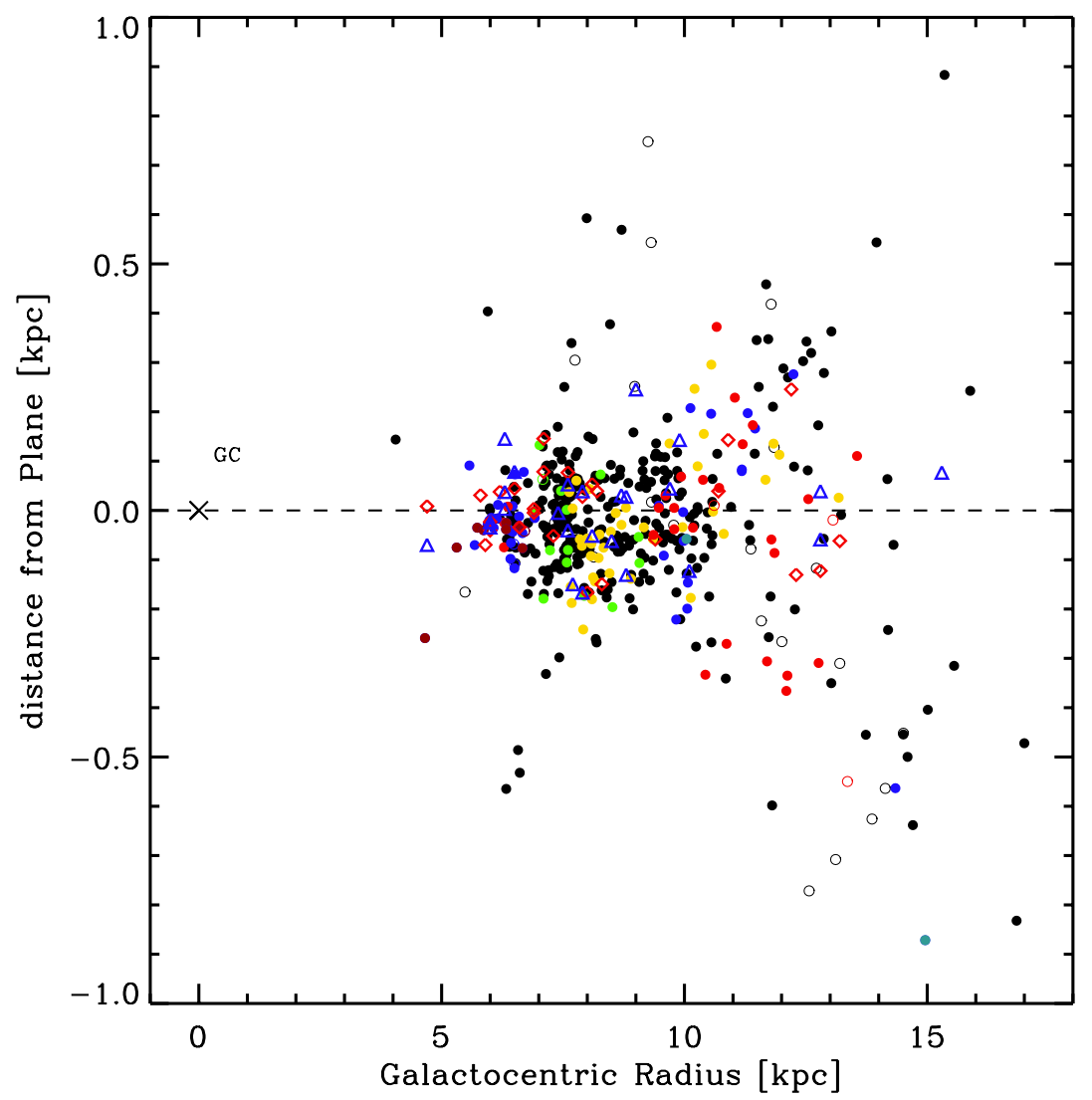

Fig. 6. Distance from the Galactic plane vs. Galactocentric distance of Galactic Cepheids with accurate iron abundances. Symbols and colors are the same as in Fig. 4; the black cross marks the position of the Galactic center (GC).
The open clusters (OCs) have several advantages as stellar tracers of the Galactic thin disk. First, they typically host a sizeable sample of RGs; this means that multi-object spectrographs can provide very accurate measurements for both iron and $\alpha$-elements. Second, their distances can be evaluated with good precision by using the main sequence fitting. Finally, they trace a significant fraction of the Galactic disk (see the WEBDA website ${ }^{5}$ ) and their ages range from several hundred Myr to several Gyrs. The main drawback is that they are affected by high reddening and quite often by differential reddening. To fully exploit the advantages of using OCs to constrain the metallicity gradient, we selected a sample of 67 OCs for which spectroscopic measurements of iron abundances are available in the literature. To provide a homogeneous metallicity scale for OCs, the individual estimates were rescaled to the solar iron abundance we adopted in this investigation. For the OCs with multiple estimates of the iron abundance in the literature, we typically adopted the most recent measurement. Columns 4 and 5 in Table 5 give both the original and the rescaled iron abundance $^{6}$, while Cols. 6 and 7 give the reference for the metallicity and for the age/distance.

In dealing with Galactocentric distances of OCs, the main source of uncertainty is the calibration of the adopted distance indicator.

Moreover and even more importantly, age estimates are tightly correlated with the adopted cluster distance, reddening, and metallicity. The cluster age also depends on the evolutionary framework (overshooting, mass loss, rotation, microphysics) adopted to compute evolutionary models and cluster isochrones (Bono et al. 2001e; Salaris \& Cassisi 2008; Prada Moroni et al. 2012; Neilson et al. 2012b; Anderson et al. 2013).

\footnotetext{
5 http://webda.physics . muni.cz/

6 In a few cases we have not been able to rescale the iron abundance since the authors did not quote the adopted solar iron abundance.
}

To overcome this thorny problem and to limit their intrinsic dispersion in the metallicity gradient we decided to use only OC with homogeneous estimates of the five crucial parameters: age, distance, reddening, abundance, theoretical framework. In particular, we selected determinations provided by Salaris et al. (2004) and Friel (1995) (30 OCs), by the Carraro group (13 OCs), by BOCCE ${ }^{7}$ (8 OCs), and by Friel \& Janes (1993) (8 OCs). We adopted the Yong et al. (2012) values for two remaining OCs. For seven OCs selected by Cheng et al. (2012) we adopted the parameters given by WEBDA and for PWM4 the estimates provided by Yong et al. (2012). The Galactocentric distances are listed in Col. 3 in Table 5 and they were calculated using the same value of the Sun Galactocentric distance $\left(R_{\mathrm{G}}=\right.$ $7.94 \mathrm{kpc})$.

The top panel of Fig. 7 shows the comparison between Cepheids (black dots) and 44 OCs younger than 3 Gyr. Diamonds show the position of OCs and different data sets are marked with different colors (see also Table 5). Data plotted in this panel show that Cepheids and OCs younger than 3 Gyr are characterized by similar trends when moving from the inner to the outer disk. The same outcome applies, within the errors, to the intrinsic dispersion.

We found a metallicity gradient for the young OCs in our sample of $[\mathrm{Fe} / \mathrm{H}]=0.47 \pm 0.10-0.051 \pm 0.010 R_{\mathrm{G}} / \mathrm{kpc}$ (see the top panel of Fig. 5), in which both the slope and the zero-point attain values very similar to the Cepheid metallicity gradient (see Fig. 4).

\subsection{Intermediate-age tracers}

The bottom panel of Fig. 7 shows the same comparison as the top panel, but for OCs (23) with ages ranging from 3.6 Gyr to 9 Gyr. Data plotted in this panel show two distinctive features; i) old

7 http://www.bo.astro.it/ angela/bocce.html 


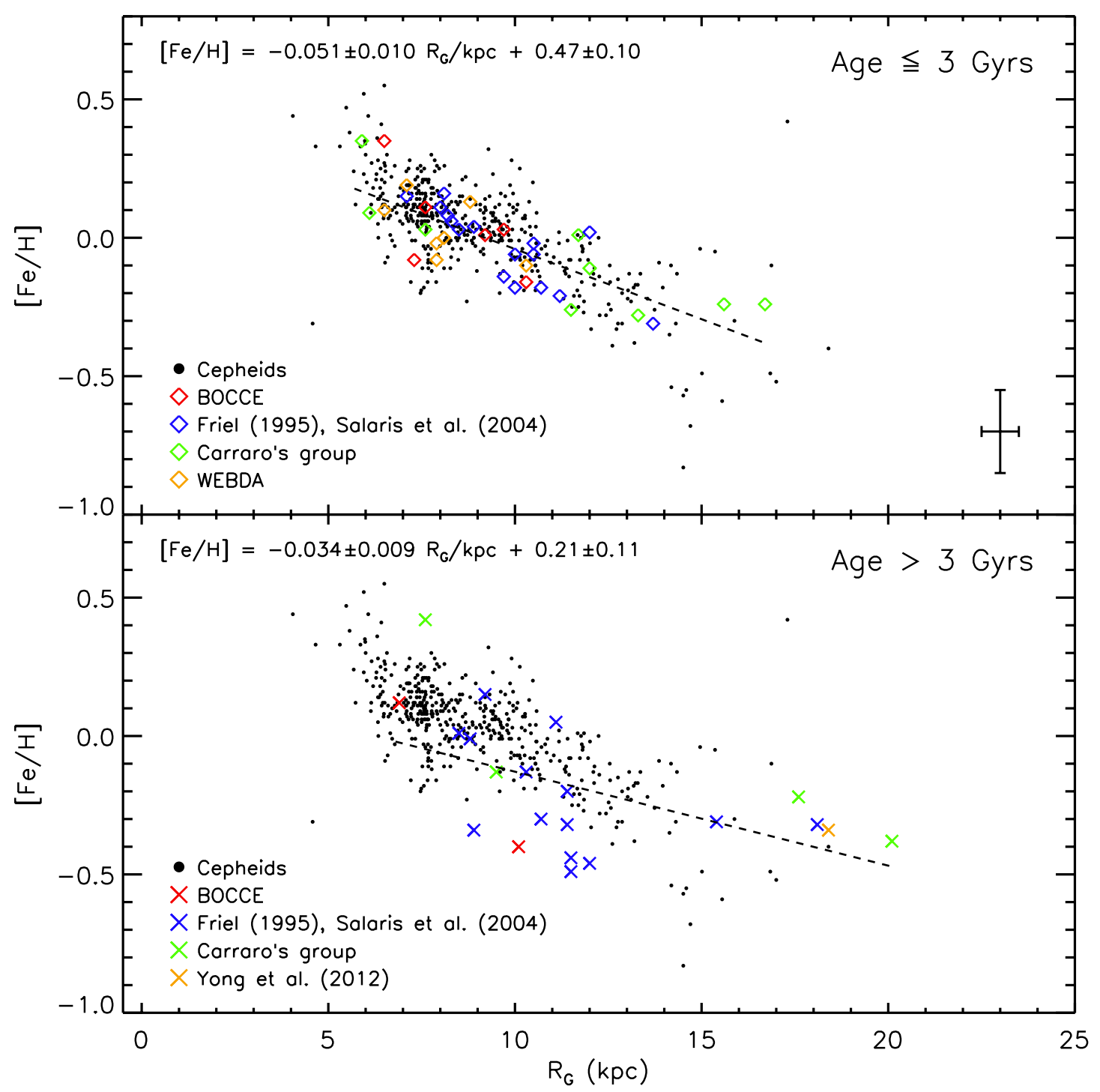

Fig. 7. Top: comparison of the Cepheid (black dots) metallicity gradient with iron abundances for open clusters younger than 3 Gyr. The color coding refers to different subsamples: BOCCE project (red); cluster ages from Salaris et al. (2004) and distances from Friel (1995) (blue); Carraro et al. (green); WEBDA (yellow). See Table 5 for individual values and references. The vertical and horizontal black error bars in the bottom right corner show the mean uncertainty on Galactocentric distances and iron abundances for OCs in the above subsamples. The dashed line shows the metallicity gradient based on the selected open clusters. Bottom: same as the top, but for cluster ages older than 3 Gyr. The yellow cross marks the position of the subsample from Yong et al. (2012). The dashed line shows the metallicity gradient based on the selected open clusters.

OCs display a clear flattening in iron abundance for $R_{\mathrm{G}} \geq 15 \mathrm{kpc}$ and ii) the old OCs for Galactocentric distances between the solar circle and $12 \mathrm{kpc}$ seem to show a dichotomic distribution. The difference is of the order of several tenths of dex, i.e., larger than possible uncertainties affecting individual iron abundances. We also checked the position of the seven OCs that are, at fixed Galactocentric distance more metal-poor and we found that five out of the seven cover a very narrow range in Galactic latitude $(y \sim 3.5 \mathrm{kpc})$. Data plotted in the above figure support the evidence that the metallicity gradient depends on age for ages older than $\sim 3$ Gyr. We could also speculate that there are a dozen OCs distributed along a metal-poor plateau with an almost constant iron abundance $([\mathrm{Fe} / \mathrm{H}] \sim-0.4)$ and Galactocentric distances ranging from $9 \mathrm{kpc}$ to $21 \mathrm{kpc}$.

We estimated the metallicity gradient of the older OCs and we found of $[\mathrm{Fe} / \mathrm{H}]=0.21 \pm 0.11-0.034 \pm 0.009 R_{\mathrm{G}} / \mathrm{kpc}($ see the bottom panel of Fig. 5). The slope and the zero-point are significantly shallower than for Cepheids and younger open clusters and agree quite well with similar estimates available in the literature for old OCs (Carraro et al. 2007b; Yong et al. 2012). However, our sample of OCs covers a range in age of five Gyr and the sample is modest. More solid constraints call for larger samples of OCs and a wider disk coverage.

However, data plotted in the bottom panel of Fig. 7 show that the Cepheid iron abundances in the inner disk are more metal-rich than thin disk old OCs. Moreover, the Cepheids display a well-defined iron gradient when moving from the inner to the outer disk ( $5 \leq R_{\mathrm{G}} \leq 18 \mathrm{kpc}$ ). However, the above evidence relies on stellar populations with significantly different ages. The Cepheids and the supergiants of the nuclear bulge and of the Bar have ages ranging from a few Myr to a few hundred Myr.

The above findings indicate that younger tracers appear to be still in situ, i.e., in the same regions where they formed, while 
the intermediate-age tracers appear to be affected both by radial gas flows and by radial migration, as suggested by chemical evolution models (Portinari \& Chiosi 2000; Curir et al. 2012; Minchev et al. 2013). However, current data do not allow us to constrain the timescale within which the metallicity gradient becomes shallower.

\section{The fine structure of the metallicity gradient}

We are facing the evidence that the intrinsic dispersion of the iron metallicity gradient is, at fixed Galactocentric distance, systematically larger than the expected standard deviation (see the error bar in the right corner of the bottom panel of Fig. 4). This circumstantial evidence stimulated several investigations aimed at constraining the physical reasons for such a broad distribution. On the basis of a large Cepheid data set Luck et al. (2006) suggested that the large dispersion in iron abundance for Galactocentric distances of $R_{\mathrm{G}} \sim 9-11 \mathrm{kpc}$ was caused by a metallicity island located at $l=130^{\circ}$. However, the detection of a well-defined region characterized by a higher iron abundance was not supported in a subsequent analysis by Luck \& Lambert (2011) by using a larger Cepheid sample. The evidence of a clumpy metallicity distribution across the Galactic disk was also brought forward by Lemasle et al. (2008) and by Pedicelli et al. (2009) by using similar samples of Galactic Cepheids. However, the evidence was partially hampered by the sample size and by the lack of a homogeneous metallicity scale.

\subsection{Identification of Cepheid groups}

To further constrain the above preliminary evidence we decided to follow a different approach. We performed a new search for Cepheids groups (CGs) across the Galactic disk. The search in 3D space follows a method originally suggested by Battinelli (1991) and applied to Galactic Cepheids by Ivanov (2008, hereafter I08). He adopted $J, H, K$ 2MASS photometry for 345 Galactic Cepheids and identified 18 CGs. The current approach when compared with I08 has several advantages: i) we are dealing with a Cepheid sample that is $30 \%$ larger and they cover more than $15 \mathrm{kpc}$ across the disk; ii) individual Cepheid distances are independent of reddening corrections; and iii) $56 \%$ of NIR Cepheid mean magnitudes are based on multi-epoch light curves.

We ranked the entire Cepheid sample and arbitrarily selected the first one as a pivot and estimated its closest neighborhood by using their rectangular coordinates $x, y$, and $z$. Then, we selected the second Cepheid as a pivot, but we removed the first one from the list. In the next step, we selected the third Cepheid in our list as a pivot, but we removed the first and the second one from the list. This process is iterated until we reach the last but one Cepheid in our list. Thus, we are left with a set of $N-1$ pair distances that provide, by definition, a path connecting the entire Cepheid sample, what (Battinelli 1991) called "Path Linkage Criterion". The two main positive features of the above algorithm are that a region with a high concentration of short pair distances is also a region in 3D space with a high concentration of Cepheids. Moreover, the use of relative distances on a common path provides solid detections of filamentary groups (Battinelli 1996).

Once we have the set of pair distances, we need to define (on the basis of our Cepheid sample) a characteristic distance called the search distance $\left(d_{\mathrm{S}}\right)$ that will allow us to identify candidate Cepheid groups. In particular, we define a candidate Cepheid group if $m$ Cepheids (with $m \geq 6$ ) have a distance

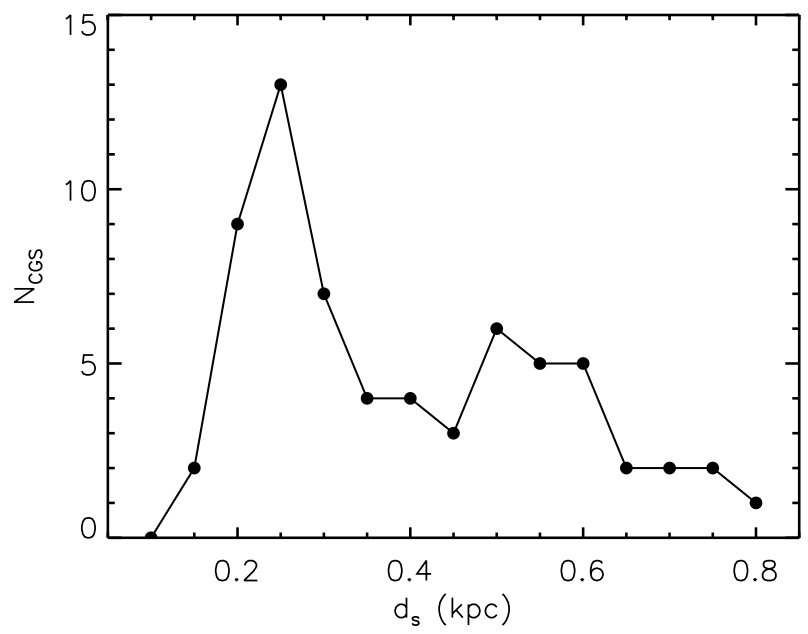

Fig. 8. Number of candidate Cepheid groups as a function of the search distance (see text for details).

$d<d_{\mathrm{S}}$, where $d_{\mathrm{S}}$ is an arbitrary distance in kpc. We adopted $d_{\mathrm{S}}$ distances ranging from $0.1 \mathrm{kpc}$ to $0.8 \mathrm{kpc}$ with a step in distance of $0.05 \mathrm{kpc}$. Figure 8 shows the number of independent CGs we detected as a function of the searching distance. The distribution of the candidate CGs we found is similar to the distribution found by I08. However, the current peak of the distribution is slightly smaller (13 vs. 18) and takes place at smaller $d_{\mathrm{S}}$ distances $(0.25 \mathrm{vs} .0 .40 \mathrm{kpc})$. Moreover, the number of candidate CGs decreases quite rapidly for $d_{\mathrm{S}}$ distances larger than $\sim 0.6 \mathrm{kpc}$ while I08 detected CGs at $d_{\mathrm{S}}$ distances larger than $1 \mathrm{kpc}$ (see Fig. 3 in I08). The difference might be explained with the difference in sample size and in the adopted Cepheid distances.

Once we have defined the optimal search distance for our Cepheid sample we need to define a criterion to constrain how significant the density of the individual candidate CG is when compared with the average stellar density of its neighborhood. In particular, we define a bonafide CG only the candidate CGs whose density $\left(\rho_{\mathrm{CG}}\right)$ is four times larger than the density of a spherical layer $\left(\rho_{\mathrm{sl}}\right)$ centered on the center of mass of the CG. The outer radius of the spherical layer $\left(R_{\mathrm{sl}}\right)$ was fixed in such a way that the volume of the spherical layer is three times larger than the volume of the CG. In particular, $V_{\mathrm{sl}}=3 V_{\mathrm{CG}}=$ $4 \pi\left(R_{\mathrm{sl}}-R_{\mathrm{CG}}\right)^{3} / 3$ where $V_{\mathrm{CG}}$ is the volume of the candidate CGs estimated by using a Monte Carlo method and by assuming for each Cepheid in the group a radius equal to the adopted search distance $\left(d_{\mathrm{S}}\right)$. The radius of the candidate $\mathrm{CG}$ was defined as $R_{\mathrm{CG}}=1.1 \times \delta$, where $\delta$ is the distance of the two most distant Cepheids. We note that $R_{\mathrm{CG}}$ is also by construction the inner radius of the spherical layer adopted to estimate the difference in density between the candidate CG and its stellar neighborhood.

Figure 9 shows a 3D graphical view of the approach we adopted to estimate the density of the candidate CGs and the density of the spherical layer. The members of the candidate Cepheid group are plotted as magenta spheres, while the inner light gray sphere defines $V_{\mathrm{CG}}$, i.e., the volume of the sphere (with radius equal to $R_{\mathrm{CG}}$ ) adopted to estimate the density of the candidate $\mathrm{CG}\left(\rho_{\mathrm{CG}}\right)$. The dark gray sphere defines $V_{\mathrm{sl}}$, i.e., the volume of the spherical layer of outer radius $R_{\mathrm{sl}}$ and inner radius $R_{\mathrm{CG}}$ adopted to estimate the average density of the stellar vicinity $\left(\rho_{\mathrm{rl}}\right)$.

In order to fix the cutoff density for the identification of CGs, we performed a series of numerical experiments in which we randomly distributed the same number of Cepheids across the 


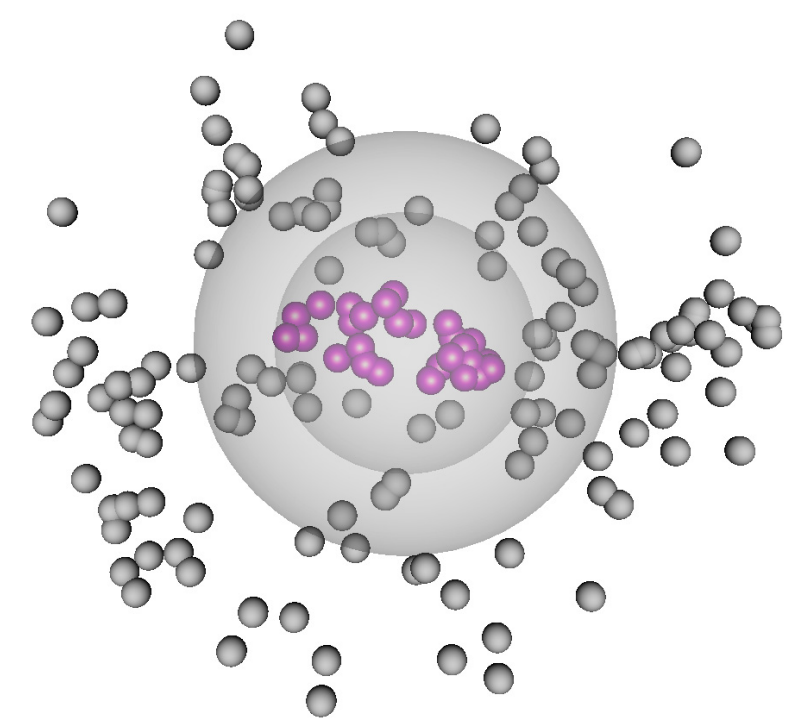

Fig. 9. 3D plot of our approach for selecting candidate CGs. The members of a generic CG are displayed with magenta spheres and the surrounding Cepheids with gray spheres. The dark gray inner area shows the sphere including the members of the CG (the radius $R_{\mathrm{CG}}$ ). The light gray outer area shows the spherical layer with a volume three times larger than the volume of the selected CG. Candidate CGs have a Cepheid density that is 3.5 larger than the average stellar density of its neighborhood.

Galactic disk and we found that their densities are systematically smaller than four times the densities of the spherical layers. By adopting this conservative selection criterion we ended up with ten candidate CGs. The overdensities of the selected CGs range from 4.1 to 45.7 when compared with their stellar neighborhood. The coordinates and the Galactocentric distances of the newly identified Cepheids are listed in Cols. 1 to 5 of Table 6 together with their diameters and the number of members. The smaller groups have on average 6-7 members and have sizes of the order of half a kpc, while the largest ones have 20-50 members and sizes between $1.2 \mathrm{kpc}$ and $1.9 \mathrm{kpc}$. The above dimensions are similar to the typical size of giant molecular clouds (see Fig. 3 in Bolatto et al. 2008 and Table 1 in Murray 2011) and to the typical size of giant star complexes and superassociations (Elmegreen \& Elmegreen 1983; Efremov 1995).

To further constrain the spatial distribution of the newly identified candidate CGs, the top panel of Fig. 10 shows their projection (filled circles) onto the Galactic plane. The members of the individual candidate CGs are plotted in yellow and confined by ellipses. Each group is marked by an increasing Roman number according to the Galactocentric radius. In order to find a correlation between the location of candidate CGs and the spiral arms, we also plotted a simplified model of the disk spiral structure. We used the logarithmic model presented by Vallée (2002) with four arms and a pitch angle of 12 degrees. The logarithmic parameter $r_{0}=2.58$ was fixed in such a way that the Perseus arm overlaps with the fiducial points of the model provided by Cordes \& Lazio (2002). We adopted this empirical calibration because $\mathrm{Xu}$ et al. (2013) found that this disk model fits quite well the parallax data of 30 masers associated with star forming regions in the Perseus and Sagittarius arms.

The true location of the spiral arms is not well defined, since it depends on the adopted tracers whose distance is quite often poorly known. However, data plotted in the top panel of Fig. 10 indicate a correlation between candidate Cepheid groups and star formation regions associated with the spiral arms.

\subsection{Residuals of the metallicity gradient}

To further investigate the physical connection of the individual members of the candidate CGs, we analyzed the residuals of the metallicity gradient. We estimated for each Cepheid in our sample the difference between its iron abundance and the iron abundance of the metallicity gradient at the same Galactocentric distance. To avoid spurious fluctuations in the mean iron abundance, we ranked all the Cepheids as a function of the Galactocentric distance $\left(R_{\mathrm{G}}\right)$ and estimated the running average by using the first 20 objects in the list. The mean $R_{\mathrm{G}}$ and the mean residual $(\Delta[\mathrm{Fe} / \mathrm{H}])$ of the bin were estimated as the mean over the individual Galactocentric distances and residual abundances of the same 20 objects. We estimated the same quantities by moving one object in the ranked list until we accounted for the last 20 Cepheids in the sample with the largest distances. The running average is plotted as a black line in the bottom panel of Fig. 10. The error on the mean residual for individual bins is of the order of a few hundredths of dex. In order to provide robust constraints on the possible uncertainties introduced by the adopted number of Cepheids per bin and by the number of stepping stars, we performed a series of Monte Carlo simulations. The estimated mean dispersion of the above simulations is plotted as a vertical black line.

Interestingly enough, the residuals display local minima and maxima that are significantly larger than the intrinsic dispersion. The occurrence of the above chemical inhomogeneities is well defined for Galactocentric distances smaller than $11 \mathrm{kpc}$. Unfortunately, the current sample does not allow us to reach firm conclusions concerning the outer disk. To constrain the nature of the secondary features in the residuals, we overplotted the position of the ten candidate CGs in the bottom panel of Fig. 10 (red dots). We adopted the mean Galactocentric distance and the mean iron abundance of the individual CGs listed in Cols. 5 and 14 in Table 6 and subtracted the iron abundance of the metallicity gradient at the same $R_{\mathrm{G}}$. The red vertical lines display the standard deviation of the iron abundances, while the horizontal red lines display the inner and the outer edge of the individual CGs (Cols. 6 and 7 in Table 6).

Data plotted in this figure show that the residuals in iron abundance appear to be tightly correlated with the mean residual abundance abundance of the candidate CGs. This finding further supports the evidence that a significant fraction of the intrinsic dispersion of the metallicity gradient is caused by the presence of Cepheid groups across the Galactic disk with mean metallicities that are either more metal-rich or more metal-poor than expected according to a linear mean metallicity gradient. The mean periods and their intrinsic dispersions listed in Cols. 10 and 11 in Table 6 seem to suggest that the candidate CGs with negative iron residuals have, on average, slightly longer periods and larger intrinsic dispersions when compared with the candidate CGs showing a positive iron residual. However, this evidence could be caused by an observational bias, and indeed the former candidate CGs have higher Cepheid densities (see Cols. 9 and 13 in Table 6) than the latter ones. The pulsation and evolutionary properties of the candidate CGs will be discussed in a forthcoming paper.

The above empirical evidence also has implications concerning the chemical enrichment of the Galactic disk. During the last few years it has been suggested that iron and oxygen abundances show a break in the abundance gradient associated 

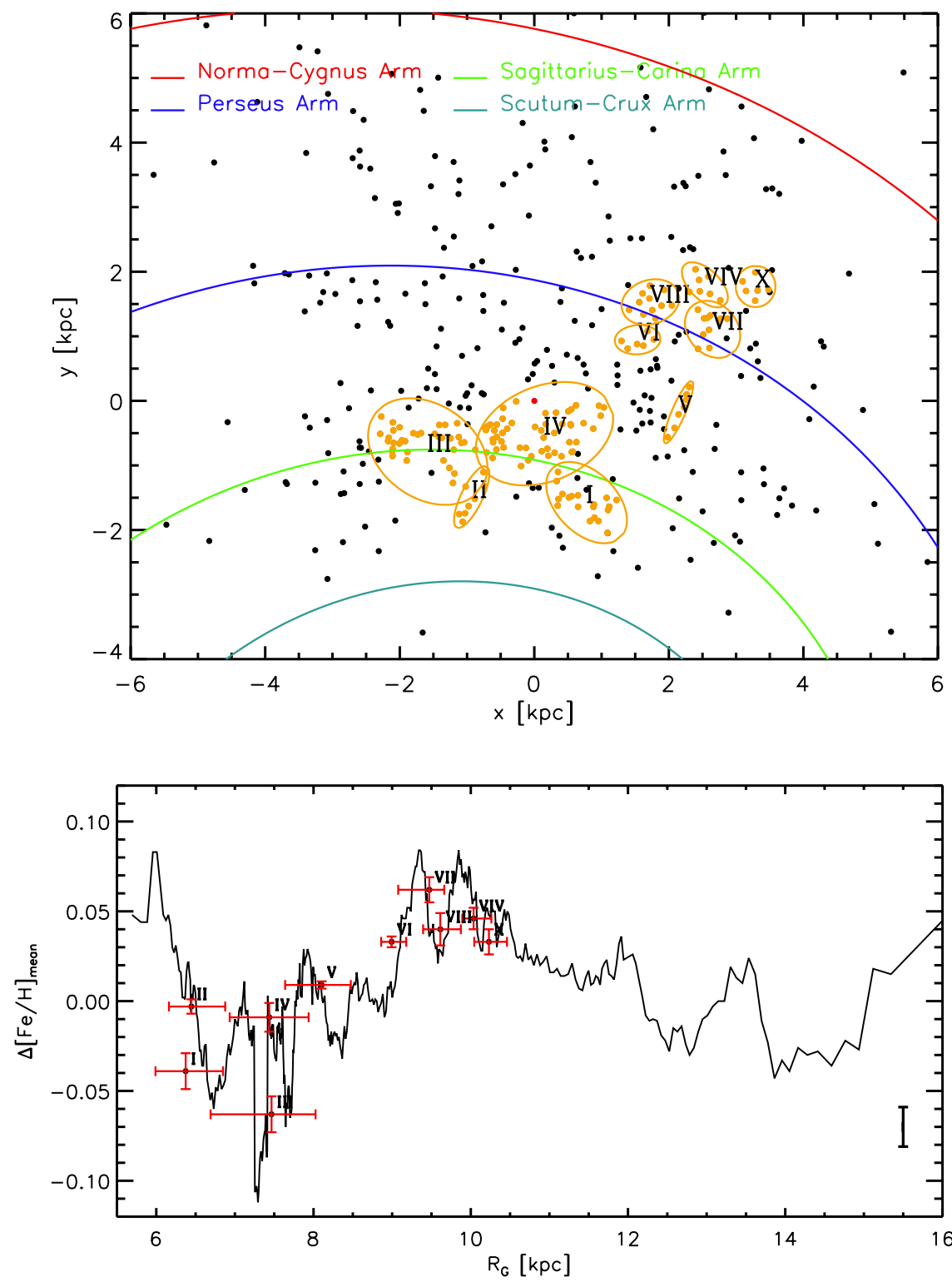

Fig. 10. Top: projection onto the Galactic plane of isolated Cepheids (black dots) and Cepheids members of candidate CGs (yellow dots). The ellipses mark the edges of individual CGs. They are also labeled with the identification number given in Table 6. The red dot shows the position of the Sun. The colored lines show a logarithmic model of the spiral arms (Vallée 2002; Cordes \& Lazio 2002, see text for details). The names of the spiral arms are labeled. Bottom: running average of Cepheid metallicity residuals vs. Galactocentric radius (black line). The red dots mark the mean metallicity of the candidate CGs once the metallicity gradient has been subtracted. The red vertical lines display the intrinsic dispersion in iron of candidate CGs. The red horizontal lines show the inner and outer Galactocentric distance of individual candidate CGs (see Cols. 6 and 7 in Table 6). with the corotation resonance of the spiral pattern (see, e.g., Acharova et al. 2010). This evidence applies not only to external spiral galaxies (Scarano et al. 2011; Scarano \& Lépine 2013), but also to our Galaxy (Lépine et al. 2011). In particular, it has been suggested that the breaks in iron, $\alpha$-elements, and barium abundance gradients of Cepheid and open cluster are caused by the corotation resonance located at $R_{\mathrm{G}} \sim 9.0-9.5 \mathrm{kpc}$ (Lépine et al. 2013). The occurrence of a discontinuity at the above Galactocentric distance is further supported by the presence of a well-defined local minimum in the Galactic rotation curve (see Figs. 1, 3, and 5 in Sofue et al. 2009). On the basis of the above preliminary evidence it has been suggested that the Galaxy is experiencing a bimodal chemical evolution, since the kinematics of the gas has opposite directions at the corotation resonance of the spiral pattern.

However, current iron abundances for Galactic Cepheids do not show a break, a jump, or a change in the slope for $R_{\mathrm{G}} \sim 9.0-9.5 \mathrm{kpc}$. Moreover and even more importantly, the positive iron residual located at the above Galactocentric distance appears to be associated with the five candidate CGs that are located across the Perseus arm (see the solid blue line in the top panel of Fig. 10). We note that the association between the candidate CGs and the Perseus arm requires a more detailed analysis, since we adopted a qualitative model of the Galactic logarithmic spiral arms (Vallée 2005). In passing, we note that current findings, once confirmed by independent stellar tracers, support the suggestion brought forward by Sofue et al. (2009) and by Sofue (2013) that the dip in the rotation curve $R_{\mathrm{G}} \sim$ 9.0-9.5 is caused by a massive ring associated with the Perseus arm.

\section{Luminosity amplitudes and metallicity dependence}

During the last few years the dependence of the luminosity amplitude on metallicity has been investigated both from the theoretical point of view (Bono et al. 2000b) and the observational (Klagyivik \& Szabados 2009; Pedicelli et al. 2010; Klagyivik et al. 2013). In particular, Szabados \& Klagyivik (2012), by using a large sample (327) of Galactic Cepheids with accurate pulsation parameters and spectroscopic metal abundances, found evidence that the luminosity and the radial velocity amplitudes slightly decrease with increasing iron abundance. However, no firm conclusion has been reached since 
Pedicelli et al. (2010), by using a similar data set, did not find a clear dependence on metallicity. More recently, Klagyivik et al. (2013) by using short-period $(\log P \leq 1.02)$ Cepheids found that the $R_{21}$ and the $R_{31}$ Fourier amplitude ratios decrease for increasing iron abundance.

To further constrain the behavior of this interesting diagnostic we took advantage of the current sample of Galactic Cepheids with homogeneous spectroscopic abundances. We limited our sample to fundamental Cepheids for which accurate $V$-band amplitudes are available ${ }^{8}$ and we ended up with a sample of 351 Cepheids. However, the period distribution of Galactic Cepheids shows a short tail in the long-period range when compared with Magellanic Cloud (MC) Cepheids (Gascoigne 1974; Bono et al. 2010; Inno et al. 2013; Genovali et al. 2013). To overcome possible biases in constraining the metallicity dependence, we took advantage of the MC Cepheids for which were available spectroscopic iron abundances (Luck \& Lambert 1992; Luck et al. 1998; Romaniello et al. 2008) and $V$-band amplitudes (SMC and LMC field Cepheids: OGLE III (Soszynski et al. 2008; Soszyñski et al. 2010), ASAS (Karczmarek et al. 2011, 2012), as well as data provided by van Genderen (1983); Freedman et al. (1985); Caldwell et al. (1986, 2001); van Genderen \& Nitihardjo (1989). For cluster Cepheids we adopted data provided by Sebo \& Wood (1995) [NGC 1850] and by Welch et al. (1991); Welch \& Stetson (1993) [NGC 1866].

We ended up with a sample of 58 Large Magellanic Cloud (LMC) and 19 Small Magellanic Cloud (SMC) Cepheids. We note that in the first sample 19 Cepheids belonging to the cluster NGC 1866 and 7 Cepheids belonging to the cluster NGC 1850 have also been included. The metallicities and the pulsation parameters for the Magellanic Cepheids are listed in Table 7. The top left panel of Fig. 11 shows the Bailey diagram ( $V$-band luminosity amplitude vs. logarithmic period) for the entire sample (428 stars). The center of the Hertzsprung progression at $\log P \leq 1.02$ is quite evident. The reader interested in a more detailed discussion concerning the nature of the Hertzsprung progression and its metallicity dependence is refereed to Bono et al. (2000a,b).

In order to constrain the dependence of the luminosity amplitude on the metallicity we split the entire sample into metal-poor $([\mathrm{Fe} / \mathrm{H}] \leq 0.03$, red circles $)$ and metal-rich $([\mathrm{Fe} / \mathrm{H}]>$ 0.03 , green circles). To avoid spurious fluctuations in the mean $A_{V}$ amplitude, we ranked all the Cepheids as a function of the logarithmic period and estimated the running average by using the first 25 objects in the list. The mean $\log P$ and the mean $A_{V}$ of the bin were estimated as the mean over the individual periods and amplitudes of the same 25 objects. We estimated the same quantities by moving one object in the ranked list until we accounted for the 25 Cepheids with the longest periods. The running averages for the metal-poor and the metal-rich samples are plotted as red and green lines in the bottom left panel of Fig. 11. The error on the mean $A_{V}$ for individual bins is of the order of a few hundredths of mag. In order to provide robust constraints on the possible uncertainties introduced by the adopted number of Cepheids per bin and by the number of stepping stars, we performed a series of Monte Carlo simulations. The estimated mean dispersions of the above

\footnotetext{
8 For a small sample of Cepheids the $V$-band luminosity amplitude was not available in the literature. For these objects the $V$-band amplitude was estimated using the amplitude relations $A_{V}=A_{I} / 0.622$ for $\log P<$ 1.02 and $A_{V}=A_{I} / 0.606$ for $\log P>1.02$ provided by Klagyivik \& Szabados (2009).
}

simulations are plotted as vertical green and red lines. We also found that we can exclude a metallicity dependence of the two subsamples at the $98 \%$ confidence level. The two running averages plotted in the bottom panel display a strong similarity in the short-period $(\log P \leq 1.02)$ range, but the difference increases at longer periods. We performed the same analysis by splitting the sample in short- and long-period Cepheids and we found that the metallicity dependence in the former group can be excluded at the $94 \%$ level, while in the latter group at the $70 \%$ level.

To further constrain the dependence of the $V$-band amplitude on metallicity, we performed the same analysis, but the entire sample was split into metal-poor $([\mathrm{Fe} / \mathrm{H}] \leq-0.10)$, metal-intermediate $(-0.10 \leq[\mathrm{Fe} / \mathrm{H}] \leq+0.10)$, and metal-rich $([\mathrm{Fe} / \mathrm{H}] \geq+0.10)$. The three different subsamples are plotted as red, black, and green circles in the top right panel of Fig. 11, while the three running averages and their mean dispersions are plotted in the bottom right panel of the same figure. The outcome concerning the metallicity dependence is quite similar to the above analysis. We found that the metallicity dependence between the metal-poor and the metal-intermediate subsamples can be excluded at the $90 \%$ confidence level, while the dependence between the metal-poor and the metal-rich subsample at the $80 \%$ level. We also split the sample in shortand long-period and we found that the dependence can be excluded at the $93 \%$ and at the $90 \%$ level between metal-poor and metal-intermediate Cepheids and at the $90 \%$ and at the $77 \%$ level between metal-poor and metal-rich Cepheids.

The above findings indicate that the luminosity amplitude of Galactic and MC Cepheids does not display a solid trend with metal abundance. However, the current analysis should be cautiously treated for two different reasons: i) the Galactic sample is still dominated by Cepheids at solar iron abundance, since the sample of more metal-poor Cepheids located in the outer disk $\left(R_{\mathrm{G}} \geq 13 \mathrm{kpc}\right)$ is quite limited and ii) the spectroscopic abundances for MC Cepheids are dominated by brighter (long-period) Cepheids. Firm conclusions concerning the metallicity dependence require larger samples of spectroscopic abundances to further constrain the difference in pulsation amplitude and in period distribution.

\section{Discussion and conclusions}

We performed accurate new measurements of iron abundances for 42 Galactic Cepheids using high-resolution, high-S/N UVES, NARVAL, and FEROS spectra. The iron abundance, for eleven Cepheids located in the inner disk, is based on multi-epoch spectra (from four to six) and their intrinsic uncertainty is smaller when compared with other Cepheids at super-solar iron content. The current sample was complemented with Cepheid iron abundances based on high-resolution spectra provided either by our group (Lemasle et al. 2007, 2008; Romaniello et al. 2008; Genovali et al. 2013) or available in the literature (Luck et al. 2011; Luck \& Lambert 2011). We ended up with a sample of 450 Cepheids. To improve the accuracy on the metallicity distribution across the disk, we estimated homogeneous and reddening-free distances by using NIR PW relations for the entire sample.

The main findings of the current iron abundance analysis are given in more detail in the following.

- We found that the metallicity gradient, based on current spectroscopic measurements, is linear with a slope of $-0.051 \pm 0.003 \mathrm{dex} / \mathrm{kpc}$, in agreement with recent studies 

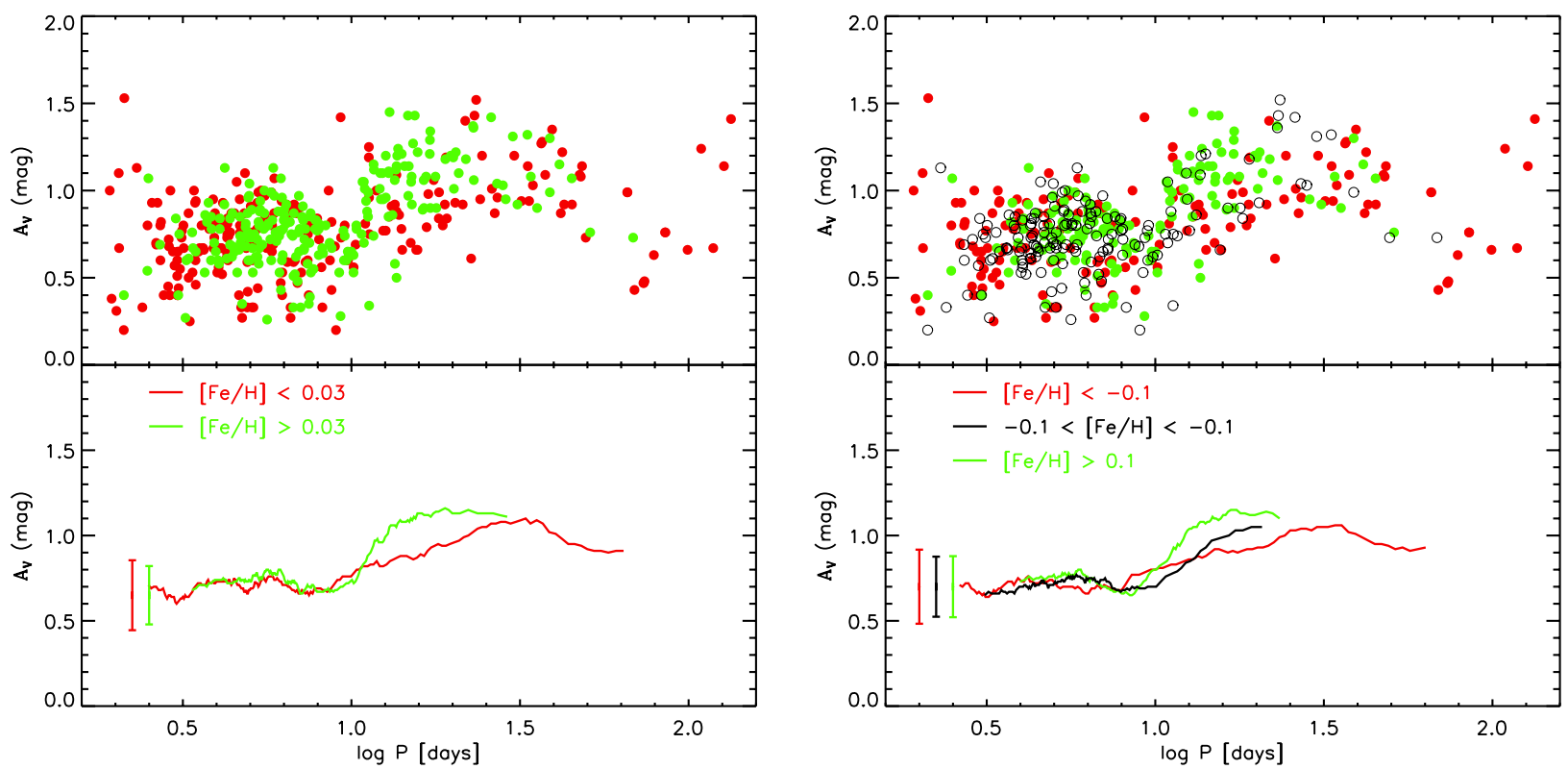

Fig. 11. Visual amplitudes vs. logarithmic period for Galactic and Magellanic Cepheids. Different colors indicate different metallicity ranges. Top left: the Cepheid sample was divided into two subsamples: supersolar (green circles) and subsolar (red) metallicity. Bottom left: running mean of the luminosity amplitudes plotted in the top panel: the color coding is the same as the top panel: the vertical bars display the intrinsic error of the running mean due to bin size and number of stepping stars (see text for more details). Top right: same as the top left panel, but the Cepheid sample was divided into three subsamples: supersolar (green), solar (empty black circles), and subsolar (red) metallicity. Bottom right: same as the bottom left panel, but for the three different metallicity bins adopted in the top panel.

by Luck et al. (2011) and Luck \& Lambert (2011). The metallicity gradient based both on our iron abundances and on those from the literature shows a similar slope: $-0.060 \pm$ $0.002 \mathrm{dex} / \mathrm{kpc}$. Current estimates agree quite well with the chemical evolution model for the thin disk recently provided by Minchev et al. (2013). In particular, they found that the iron gradient is $-0.061 \mathrm{dex} / \mathrm{kpc}$ for Galactocentric distances ranging from $5 \mathrm{kpc}$ to $12 \mathrm{kpc}$ and $-0.057 \mathrm{dex} / \mathrm{kpc}$ for Galactocentric distances ranging from $6 \mathrm{kpc}$ to $11 \mathrm{kpc}$. The predicted slopes become marginally shallower if they account for stellar radial migrations.

- We estimated the metallicity gradient by selecting the Cepheids in our sample with a distance above the Galactic plane smaller than $300 \mathrm{pc}$ and we found that it is, within the errors, quite similar: $-0.052 \pm 0.004 \mathrm{dex} / \mathrm{kpc}$. The same outcome applies to the the gradient based on the entire sample, and we found: $-0.055 \pm 0.002 \mathrm{dex} / \mathrm{kpc}$. We also found that the spread in iron in the outer disk $\left(R_{\mathrm{G}} \geq 13 \mathrm{kpc}\right)$ decreases by more than a factor of two ( 0.13 vs. $0.17 \mathrm{dex})$ if we adopt the subsample located closer to the Galactic plane.

- We also confirm that classical Cepheids in the inner disk $\left(R_{\mathrm{G}} \sim 5.5-6.0 \mathrm{kpc}\right)$, just beyond the position of the Galactic bar corotation resonance (Gerhard et al. 2011), attain super-solar $([\mathrm{Fe} / \mathrm{H}] \sim 0.4)$ iron abundances. This result supports similar findings by G13 and by Andrievsky et al. (2002); Pedicelli et al. (2010); Luck \& Lambert (2011).

There is preliminary evidence that the iron abundance in the innermost Galactic regions (nuclear bulge, Galactic bar) is more metal-poor than predicted by chemical evolution models (Minchev et al. 2013). Recent spectroscopic iron abundances of young stars (red supergiants, luminous blue variables, Wolf-Rayet, O-type stars) indicate either solar or subsolar abundances (Davies et al. 2009a,b; Origlia et al. 2013). On the other hand, chemical evolution models suggest in the same regions iron abundances larger than $[\mathrm{Fe} / \mathrm{H}] \sim 0.8$ (Minchev et al. 2013). The above evidence indicates that objects located inside the corotation resonance of the bar experienced a different chemical enrichment history than the Cepheids located just beyond this limit.

- The new homogeneous Cepheid metallicity distribution is characterized by a smaller intrinsic dispersion when compared with similar estimates available in the literature. We found evidence of a steady increase in the abundance dispersion when moving in the outer disk $\left(R_{\mathrm{G}}>14 \mathrm{kpc}\right)$. Current data do not allow us to constrain whether this effect is the aftermath of outward stellar migrators as recently suggested by Minchev et al. (2012) or the consequence of the infall of the Sagittarius dwarf galaxy producing a flared outer disk as suggested by Purcell et al. (2011).

- To investigate the fine structure of the metallicity in the disk, we searched for Cepheids groups following the approach suggested by Ivanov (2008). We found ten candidate Cepheids groups, i.e., physical aggregations of stars whose mean residual metallicity agrees quite well with the trend of the metallicity residuals as a function of the Galactocentric distance. The presence of the CGs appears to be the main culprit of the fluctuations in the metallicity residuals and of the azimuthal effects on the radial gradient. This suggests that members of CGs experienced a very similar chemical enrichment history. Most of the CGs are located close to spiral arms (Sagittarius-Carina and Perseus arms) according to a simple logarithmic spiral model provided by Vallée (2005). The above findings indicate that the occurrence of CGs with sizes ranging from $\mathrm{OB}$ association/young cluster to star complexes/superassociations appear to be largely responsible for the intrinsic spread of the iron metallicity gradient. Moreover, the association of the metallicity residuals with candidate CGs supports the results by Sofue (2013) concerning the association of a local minimum in the Galactic rotational curve at $R_{\mathrm{G}} \sim=9.5 \mathrm{kpc}$ with the Perseus arm. 
- We also found that the mean periods of the Cepheids hosted in candidate CGs with negative iron residuals have, on average, slightly longer periods and larger intrinsic dispersions when compared with the candidate CGs showing a positive iron residual, thus suggesting a common star formation episode within each candidate CG. The evidence of possible abundance inhomogeneities in the Galactic disk dates back to (Efremov 1995, and references therein) who suggested that the different star complexes might have different star formation histories and different interactions with the intergalactic medium. It is clear that the abundance information (iron and $\alpha$-elements) will provide a new spin to the analysis of their evolutionary and pulsation properties.

- To constrain the impact of age on the iron abundance gradient, we compared the Cepheid iron gradient with those based on OCs. Spectroscopic metallicities and homogeneous distances and ages were collected for OCs spanning a large range in age. The OC gradient based on clusters younger than 3 Gyr agrees quite well with the Cepheid gradient.

- The comparison between Cepheids and OCs older than 3 Gyr is more complex. We found that old OCs display a clear flattening in iron abundance for $R_{\mathrm{G}} \geq 15 \mathrm{kpc}$. This result supports similar findings available in the literature e.g., Carraro et al. (2007b), Bragaglia et al. (2008), Magrini et al. (2009, 2010), Jacobson et al. (2011a,b), Yong et al. (2012). Moreover, old OCs located between the solar circle and $R_{\mathrm{G}} \sim 12 \mathrm{kpc}$ seem to show a dichotomic distribution. The difference is of the order of several tenths of dex and might be due to a selection bias affecting the azimuthal distribution. However, the comparison of Cepheid iron abundances with similar abundances for old OCs further supports the evidence that the metallicity gradient depends on age for ages larger than $\sim 3$ Gyr.

- We investigate the possible occurrence of a metallicity effect on the pulsational amplitude by using a large sample of fundamental Galactic and Magellanic Cepheids (Luck \& Lambert 1992; Luck et al. 1998; Romaniello et al. 2008) with accurate iron abundances. The comparison of low, medium, and high metallicity subsamples indicates that luminosity amplitudes are, within current uncertainties, independent of iron abundance.

Classical Cepheids appear to be solid young stellar tracers to constrain the recent chemical enrichment of the Galactic thin disk. The current sample of Galactic Cepheids is smaller when compared with similar tracers (OB stars, HII regions, red clump stars, open clusters). However, their distances, ages, and abundances can be estimated with certainty. They are ubiquitous in young star forming regions and the recent identification of classical Cepheids both in the nuclear bulge and in the Galactic bar (Matsunaga et al. 2011, 2013) will provide the opportunity to use the same stellar tracer to constrain the change in iron abundance across the corotation resonance. This also means there is the opportunity to constrain whether the high star formation rate of the innermost Galactic regions is driven by a disk instability that is dragging material from the inner disk into these regions (Freeman et al. 2013; Ness et al. 2013a,b).

Classical Cepheids are also excellent tracers for constraining the speed of the spiral arm pattern by fitting a kinematic model to the observed Cepheid kinematics (Fernández et al. 2001; Lépine et al. 2001). The Cepheid kinematics is time consuming, since a proper coverage of the radial velocity curves does require spectroscopic time series data. The use of template radial velocity curves significantly decreases the number of measurements required for an accurate estimate of the center of mass radial velocity (Metzger et al. 1998). However, we still lack accurate radial velocity curve templates covering the entire period range.

The current observational scenario appears to be even more appealing in the outer disk, since we are still facing a "Cepheid desert" for Galactocentric distances larger than $\sim 18 \mathrm{kpc}$. New identification and characterization of Cepheids at least in the first and in the second quadrant are urgently needed to properly trace the outskirts of the Galactic disk.

Acknowledgements. It is a pleasure to thank M. Zoccali and N. Suntzeff for many interesting discussions concerning the bulge and the thin disk metallicity gradients. We also acknowledge an anonymous referee for his/her pertinent suggestions that improved the content and the readability of the paper. We are very grateful to VLT staff astronomers for transforming the original observing proposals into a solid experiment. This work was partially supported by PRIN-INAF 2011 "Tracing the formation and evolution of the Galactic halo with VST" (P.I.: M. Marconi) and by PRIN-MIUR (2010LY5N2T) "Chemical and dynamical evolution of the Milky Way and Local Group galaxies" (P.I.: F. Matteucci). One of us (K.G.) thank the ESO for support as science visitor, G.B. thanks The Carnegie Observatories visitor programme for support as science visitor. This research made use of spectra obtained from the ESO Science Archive Facility. This publication makes use of data products from the Two Micron All Sky Survey, which is a joint project of the University of Massachusetts and the Infrared Processing and Analysis Center/California Institute of Technology, funded by the National Aeronautics and Space Administration and the National Science Foundation. This research has made use of the WEBDA database, operated at the Institute for Astronomy of the University of Vienna.

\section{References}

Acharova, I. A., Lépine, J. R. D., Mishurov, Y. N., et al. 2010, MNRAS, 402, 1149

Anderson, R. I., Eyer, L., \& Mowlavi, N. 2013, MNRAS 434, 2238

Andrievsky, S. M., Kovtyukh, V. V., Luck, R. E., et al. 2002, A\&A, 392, 491

Andrievsky, S. M., Luck, R. E., Martin, P., \& Lépine, J. R. D. 2004, A\&A, 413, 159

Andrievsky, S. M., Luck, R. E., \& Kovtyukh, V. V. 2005, AJ, 130, 1880

Ballester, P., Bramich, D., Forchi, V., et al. 2011, Astronomical Data Analysis Software and Systems XX, eds. I. N. Evans, A. Accomazzi, D. J., Mink, \& A. H., Rots, ASP Conf. Ser., 442, 261

Battinelli, P. 1991, A\&A, 244, 69

Battinelli, P., Efremov, Y., \& Magnier, E. A. 1996, A\&A, 314, 51

Bolatto, A. D., Leroy, A. K., Rosolowsky, E., et al. 2008, ApJ, 686, 948

Bono, G., Castellani, V., \& Marconi, M. 2000a, ApJ, 529, 293

Bono, G., Marconi, M., \& Stellingwerf, R. F. 2000b, A\&A, 360, 245

Bono, G., Gieren, W. P., Marconi, M., Fouqué, P., \& Caputo, F. 2001, ApJ, 563, 319

Bono, G., Caputo, F., Marconi, M., \& Musella, I. 2010, ApJ, 715, 277

Bono, G., et al. 2013, in CTIO 50 years - Fifty Years of Wide Field Studies in the Southern Hemisphere, eds. A. Kunder, \& S. Points (San Francisco, ASPCS), in press

Bragaglia, A., Carretta, E., Gratton, R. G., et al. 2001, AJ, 121, 327

Bragaglia, A., Tosi, M., Carretta, E., et al. 2006, MNRAS, 366, 1493

Bragaglia, A., Sestito, P., Villanova, S., et al. 2008, A\&A, 480, 79

Brown, J. A., Wallerstein, G., Geisler, D., \& Oke, J. B. 1996, AJ, 112, 1551

Caldwell, J. A. R., Coulson, I. M., Jones, J. H. S., Black, C. A., \& Feast, M. W. 1986, MNRAS, 220, 671

Caldwell, J. A. R., Coulson, I. M., Dean, J. F., \& Berdnikov, L. N. 2001, J. Astron. Data, 7, 4

Caputo, F., Marconi, M., Musella, I., \& Pont, F. 2001, A\&A, 372, 544

Carraro, G., Girardi, L., \& Marigo, P. 2002, MNRAS, 332, 705

Carraro, G., Bresolin, F., Villanova, S., et al. 2004, AJ, 128, 1676

Carraro, G., Méndez, R. A., \& Costa, E. 2005a, MNRAS, 356, 647

Carraro, G., Geisler, D., Baume, G., Vázquez, R., \& Moitinho, A. 2005b, MNRAS, 360, 655

Carraro, G., Geisler, D., Moitinho, A., Baume, G., \& Vázquez, R. A. 2005c, A\&A, 442, 917

Carraro, G., de La Fuente Marcos, R., Villanova, S., et al. 2007a, A\&A, 466, 931

Carraro, G., Geisler, D., Villanova, S., Frinchaboy, P. M., \& Majewski, S. R. 2007b, A\&A, 476, 217

Carrera, R., \& Pancino, E. 2011, A\&A, 535, A30

Carretta, E., Bragaglia, A., Gratton, R. G., \& Tosi, M. 2004, A\&A, 422, 951 
Carretta, E., Bragaglia, A., Gratton, R. G., \& Tosi, M. 2005, A\&A, 441, 131 Carretta, E., Bragaglia, A., \& Gratton, R. G. 2007, A\&A, 473, 129 Cescutti, G., Matteucci, F., François, P., \& Chiappini, C. 2007, A\&A, 462, 943 Cheng, J. Y., Rockosi, C. M., Morrison, H. L., et al. 2012, ApJ, 746, 149 Cordes, J. M., \& Lazio, T. J. W. 2002 [arXiv:astro-ph/0207156] Curir, A., Lattanzi, M. G., Spagna, A., et al. 2012, A\&A, 545, 133 Davies, B., Origlia, L., Kudritzki, R.-P., et al. 2009a, ApJ, 694, 46 Davies, B., Origlia, L., Kudritzki, R.-P., et al. 2009b, ApJ, 696, 2014 Efremov, Y. N. 1995, AJ, 110, 2757

Elmegreen, B. G., \& Elmegreen, D. M. 1983, MNRAS, 203, 31 Fernández, D., Figueras, F., \& Torra, J. 2001, A\&A, 372, 833

Ford, A., Jeffries, R. D., \& Smalley, B. 2005, MNRAS, 364, 272 Freeman, K., \& Bland-Hawthorn, J. 2002, ARA\&A, 40, 487 Freeman, K., Ness, M., Wylie-de-Boer, E., et al. 2013, MNRAS, 428, 3660 Freedman, W. L., Grieve, G. R., \& Madore, B. F. 1985, ApJS, 59, 311 Friel, E. D. 1995, ARA\&A, 33, 381

Friel, E. D., \& Janes, K. A. 1993, A\&A, 267, 75

Friel, E. D., Jacobson, H. R., \& Pilachowski, C. A. 2005, AJ, 129, 2725 Friel, E. D., Jacobson, H. R., \& Pilachowski, C. A. 2010, AJ, 139, 1942 Gascoigne, S. C. B. 1974, MNRAS, 166, 25

Geisler, D., Villanova, S., Carraro, G., et al. 2012, ApJ, 756, L40 Genovali, K., Lemasle, B., Bono, G., et al. 2013, A\&A, 554, A132 (Paper VI) Gerhard, O. 2011, Mem. Soc. Astron. It. Supp., 18, 185

Girardi, L., \& Salaris, M. 2001, MNRAS, 323, 109

Gratton, R. G., \& Contarini, G. 1994, A\&A, 283, 911

Grevesse, N., Noels, A., \& Sauval, A. J. 1996, ASP Conf. Ser. 99, eds. S. S. Holt, \& G. Sonnborn, 117

Groenewegen, M. A. T. 2013, A\&A, 550, A70

Groenewegen, M. A. T., Udalski, A., \& Bono, G. 2008, A\&A, 481, 441 Gustafsson, B., Edvardsson, B., Eriksson, K., et al. 2008, A\&A, 486, 951 Henry, R. B. C., Kwitter, K. B., Jaskot, A. E., et al. 2010, ApJ, 724, 748 Inno, L., Matsunaga, N., Bono, G., et al. 2013, ApJ, 764, 84 Ivanov, G. R. 2008, Bulgar. Astron. J., 10, 15

Jacobson, H. R., Friel, E. D., \& Pilachowski, C. A. 2007, AJ, 134, 1216 Jacobson, H. R., Friel, E. D., \& Pilachowski, C. A. 2008, AJ, 135, 2341 Jacobson, H. R., Friel, E. D., \& Pilachowski, C. A. 2009, AJ, 137, 4753 Jacobson, H. R., Friel, E. D., \& Pilachowski, C. A. 2011a, AJ, 141, 58 Jacobson, H. R., Pilachowski, C. A., \& Friel, E. D. 2011b, AJ, 142, 59 Kalirai, J. S., \& Tosi, M. 2004, MNRAS, 351, 649

Karczmarek, P., Dziembowski, W. A., Lenz, P., Pietrukowicz, P., \& Pojmański, G. 2011, Acta Astron., 61, 303

Karczmarek, P., Dziembowski, W. A., Lenz, P., Pietrukowicz, P., \& Pojmanski, G. 2012, Acta Astron., accepted [arXiv: 1201.0790]

Klagyivik, P., \& Szabados, L. 2009, A\&A, 504, 959

Klagyivik, P., Szabados, L., Szing, A., Leccia, S., \& Mowlavi, N. 2013, MNRAS, 434, 2418

Koen, C., Marang, F., Kilkenny, D., \& Jacobs, C. 2007, MNRAS, 380, 1433

Kovtyukh, V. V., \& Gorlova, N. I. 2000, A\&A, 358, 587

Kraft, R. P., \& Schmidt, M. 1963, ApJ, 137, 249

Laney, C. D., \& Stobie, R. S. 1992, A\&AS, 93, 93

Lemasle, B., François, P., Bono, G., et al. 2007, A\&A, 467, 283 (Paper I)

Lemasle, B., François, P., Piersimoni, A., et al. 2008, A\&A, 490, 623 (Paper III)

Lemasle, B., François, P., Genovali, K., et al. 2013, A\&A, 558, A31 (Paper VII)

Lépine, J. R. D., Mishurov, Y. N., \& Dedikov, S. Y. 2001, ApJ, 546, 234

Lépine, J. R. D., Cruz, P., Scarano, S., Jr., et al. 2011, MNRAS, 417, 698

Lepine, J. R. D., Andrievky, S., Barros, D. A., Junqueira, T. C., \& Scarano, S., Jr 2013 [arXiv: 1307.7781$]$

Luck, R. E., \& Lambert, D. L. 1992, ApJS, 79, 303

Luck, R. E., \& Lambert, D. L. 2011, AJ, 142, 136

Luck, R. E., Moffett, T. J., Barnes, T. G., III, \& Gieren, W. P. 1998, AJ, 115, 605

Luck, R. E., Kovtyukh, V. V., \& Andrievsky, S. M. 2006, AJ, 132, 902

Luck, R. E., Andrievsky, S. M., Kovtyukh, V. V., Gieren, W., \& Graczyk, D. 2011, AJ, 142, 51

Maciel, W. J., Costa, R. D. D., \& Uchida, M. M. M. 2003, A\&A, 397, 667

Magrini, L., Sestito, P., Randich, S., \& Galli, D. 2009, A\&A, 494, 95

Magrini, L., Randich, S., Zoccali, M., et al. 2010, A\&A, 523, A11
Martin, W. L., \& Warren, P. R. 1979, South African Astron. Obs. Circ., 1, 98 Martins, F., Hillier, D. J., Paumard, T., et al. 2008, A\&A, 478, 219

Matsunaga, N., Kawadu, T., Nishiyama, S., et al. 2011, Nature, 477, 188

Matsunaga, N., Feast, M. W., Kawadu, T., et al. 2013, MNRAS, 429, 385

Metzger, M. R., Caldwell, J. A. R., \& Schechter, P. L. 1998, AJ, 115, 635

Minchev, I., Famaey, B., Quillen, A. C., et al. 2012, A\&A, 548, A127

Minchev, I., Chiappini, C., \& Martig, M. 2013, A\&A, 558, A9

Moitinho, A., Carraro, G., Baume, G., \& Vázquez, R. A. 2006, A\&A, 445, 493

Monson, A. J., \& Pierce, M. J. 2011, ApJS, 193, 12

Mucciarelli, A., Cristallo, S., Brocato, E., et al. 2011, MNRAS, 413, 837

Murray, N. 2011, ApJ, 729, 133

Najarro, F., Figer, D. F., Hillier, D. J., Geballe, T. R., \& Kudritzki, R. P. 2009, ApJ, 691, 1816

Neilson, H. R., Engle, S. G., Guinan, E., et al. 2012, ApJ, 745, L32

Ness, M., Freeman, K., Athanassoula, E., et al. 2013a, MNRAS, 430, 836

Ness, M., Freeman, K., Athanassoula, E., et al. 2013b, MNRAS, 432, 209

Nieva, M.-F., \& Przybilla, N. 2012, A\&A, 539, A143

Nieva, M.-F., \& Simón-Díaz, S. 2011, A\&A, 532, A2

Nordström, B., Mayor, M., Andersen, J., et al. 2004, A\&A, 418, 989

Origlia, L., Oliva, E., Maiolino, R., et al. 2013, A\&A, 560, A46

Pancino, E., Carrera, R., Rossetti, E., \& Gallart, C. 2010, A\&A, 511, A56

Pedicelli, S., Bono, G., Lemasle, B., et al. 2009, A\&A, 504, 86 (Paper IV)

Pedicelli, S., Lemasle, B., Groenewegen, M., et al. 2010, A\&A, 518, A11 (Paper V)

Portinari, L., \& Chiosi, C. 2000, A\&A, 355, 929

Prada Moroni, P. G., Gennaro, M., Bono, G., et al. 2012, ApJ, 749, 108

Przybilla, N., Nieva, M.-F., \& Butler, K. 2011, J. Phys. Conf. Ser., 328, 2015

Purcell, C. W., Bullock, J. S., Tollerud, E. J., et al, 2011, Nature, 477, 301

Romaniello, M., Primas, F., Mottini, M., et al., 2008, A\&A, 488, 747 (Paper II)

Salaris, M., \& Cassisi, S. 2008, A\&A, 487, 1075

Salaris, M., \& Girardi, L. 2002, MNRAS, 337, 332

Salaris, M., Weiss, A., \& Percival, S. M. 2004, A\&A, 414, 163

Santos, N. C., Lovis, C., Pace, G., Melendez, J., \& Naef, D. 2009, A\&A, 493, 309

Samus, N. N., Durlevich, O. V., et al. 2009, VizieR Online Data Catalog, 1, 2025 Scarano, S., \& Lépine, J. R. D. 2013, MNRAS, 428, 625

Scarano, S., Jr., Lépine, J. R. D., \& Marcon-Uchida, M. M. 2011, MNRAS, 412, 1741

Sebo, K. M., \& Wood, P. R. 1995, ApJ, 449, 164

Sestito, P., Bragaglia, A., Randich, S., et al. 2008, A\&A, 488, 943

Skrutskie, M. F., Cutri, R. M., Stiening, R., et al. 2006, AJ, 131, 1163

Sofue, Y. 2013, PASJ, 65, 118

Sofue, Y., Honma, M., \& Omodaka, T. 2009, PASJ, 61, 227

Soszyński, I. Gieren, W., Pietrzyśki, G., et al. 2005, PASP, 117, 830

Soszynski, I., Poleski, R., Udalski, A., et al. 2008, Acta Astron., 58, 163

Soszyñski, I., Poleski, R., Udalski, A., et al. 2010, Acta Astron., 60, 17

Spite, M. 1967, Ann. Astrophys., 30, 211

Storm, J., Gieren, W., Fouqué, P., et al. 2011a, A\&A, 534, A94

Szabados, L., \& Klagyivik, P. 2012, A\&A, 537, A81

Sziládi, K., Vinkó, J., Poretti, E., Szabados, L., \& Kun, M. 2007, A\&A, 473, 579

Twarog, B. A., Ashman, K. M., \& Anthony-Twarog, B. J. 1997, AJ, 114, 2556 van Genderen, A. M. 1983, A\&AS, 52, 423

van Genderen, A. M., \& Nitihardjo, G. H. 1989, A\&A, 221, 230

Vallée, J. P. 2002, ApJ, 566, 261

Vallée, J. P. 2005, AJ, 130, 569

Villanova, S., Carraro, G., Bresolin, F., \& Patat, F. 2005, AJ, 130, 652

Villanova, S., Baume, G., \& Carraro, G. 2007, MNRAS, 379, 1089

Villanova, S., Carraro, G., \& Saviane, I. 2009, A\&A, 504, 845

Welch, D. L., \& Stetson, P. B. 1993, AJ, 105, 1813

Welch, D. L., Cote, P., Fischer, P., Mateo, M., \& Madore, B. F. 1991, AJ, 101, 490

Wils, P., \& Greaves, J. 2004, Inform. Bull. Variable Stars, 5512, 1

Xu, Y., Li, J. J., Reid, M. J., et al. 2013, ApJ, 769, 15

Yong, D., Carney, B. W., Teixera de Almeida, M. L., \& Pohl, B. L. 2006, AJ, 131,2256

Yong, D., Carney, B. W., \& Friel, E. D. 2012, AJ, 144, 95 


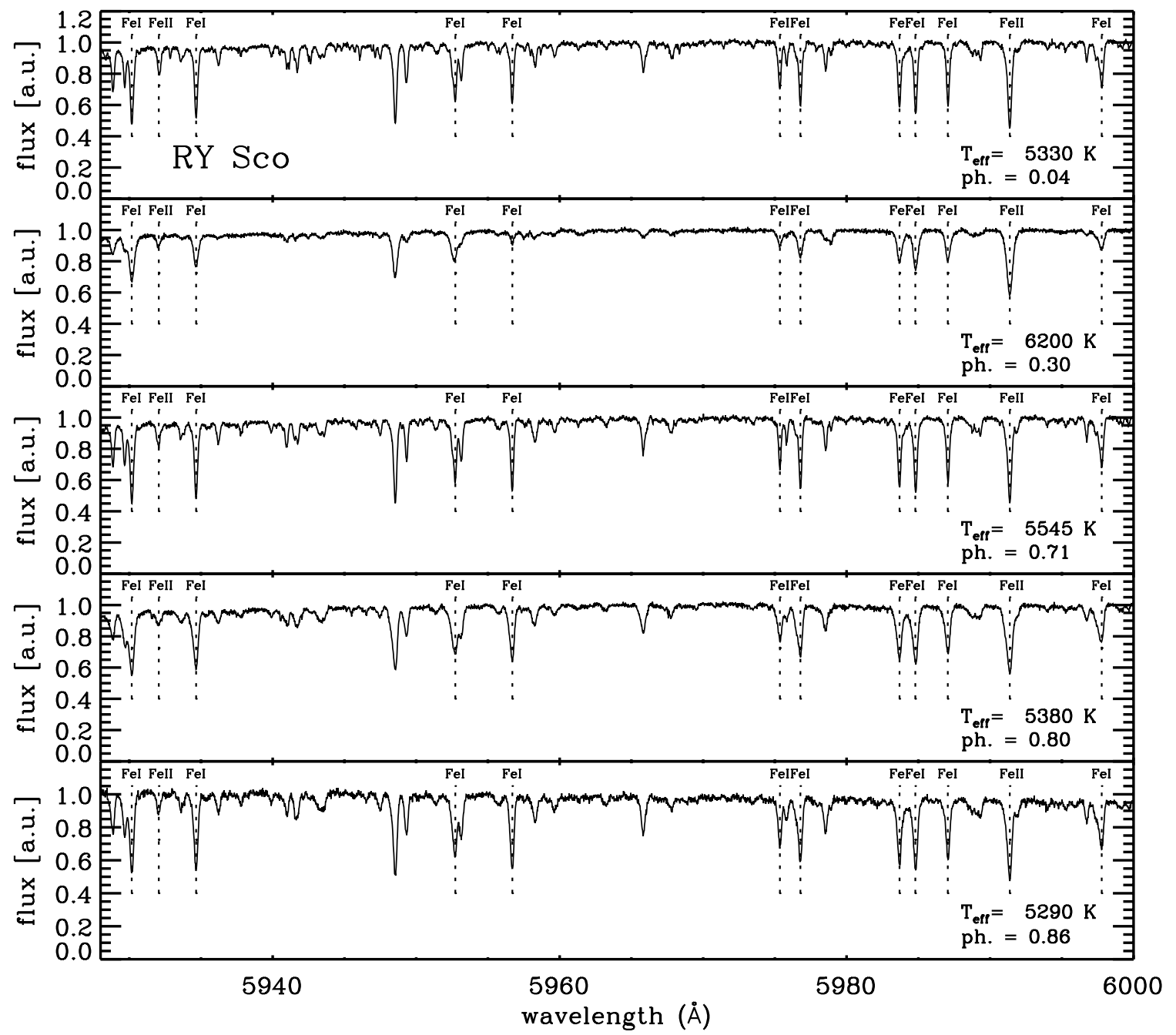

Fig. 1. High-resolution $(R \sim 38000)$ UVES spectra of solar metallicity Galactic Cepheid RY Sco $([\mathrm{Fe} / \mathrm{H}]=0.01 \pm 0.06)$. From top to bottom the spectra were plotted for increasing pulsational phase (see the corresponding $T_{\text {eff }}$ curve in Fig. 3 ). The $\mathrm{S} / \mathrm{N}$ of this sample is around 100 in the spectral range $\lambda \sim 5650-7500 \AA$. The vertical dashed lines display selected Fe I $(\lambda \lambda 5930.17,5934.66,5952.73,5956.7,5975.35,5976.78$,

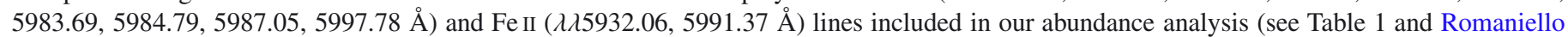
et al. 2008). 
K. Genovali et al.: The fine structure of the metallicity gradient

Table 1. Intrinsic parameters and abundances for the current sample of classical Cepheids based on individual spectra.

\begin{tabular}{|c|c|c|c|c|c|c|c|c|c|c|c|c|}
\hline Name & Date & MJD & $\begin{array}{r}E T \\
\text { (s) }\end{array}$ & $S / N$ & $\begin{array}{c}T_{\text {eff }} \\
\mathrm{K}\end{array}$ & $\log g$ & $\begin{array}{c}v_{\mathrm{t}} \\
\left(\mathrm{km} \mathrm{s}^{-1}\right)\end{array}$ & Fe I & $N$ & Fe II & $N$ & Spect. \\
\hline V340 Ara & 2012-Jul.-29 & 56137.137 & 120 & 99 & $5210 \pm 49$ & 0.30 & 5.0 & $0.28 \pm 0.27$ & 23 & $0.27 \pm 0.11$ & 2 & UVES \\
\hline V340 Ara & 2008-Aug.-30 & 54708.065 & 300 & 241 & $5210 \pm 88$ & 0.00 & 3.0 & $0.53 \pm 0.15$ & 53 & $0.53 \pm 0.11$ & 4 & UVES \\
\hline V340 Ara & 2008-Aug.-31 & 54709.079 & 300 & 264 & $5130 \pm 96$ & 0.40 & 3.0 & $0.42 \pm 0.22$ & 26 & $0.65 \pm 0.24$ & 3 & UVES \\
\hline V340 Ara & 2012-Jul.-30 & 56138.094 & 120 & 122 & $5610 \pm 68$ & 0.70 & 4.4 & $0.30 \pm 0.20$ & 41 & $0.33 \pm 0.10$ & 2 & UVES \\
\hline V340 Ara & 2012-Jul.-31 & 56139.185 & 120 & 114 & $6060 \pm 65$ & 0.80 & 5.2 & $0.24 \pm 0.13$ & 51 & $0.22 \pm 0.01$ & 2 & UVES \\
\hline V340 Ara & 2012-Aug.-13 & 56152.054 & 121 & 69 & $4820 \pm 53$ & 1.00 & 4.4 & $0.20 \pm 0.23$ & 15 & $0.30 \pm 0.29$ & 2 & UVES \\
\hline AS Aur & 2009-Jan.-14 & 54845.136 & 500 & 94 & $5800 \pm 86$ & 1.10 & 2.6 & $0.01 \pm 0.14$ & 74 & $-0.01 \pm 0.10$ & 8 & UVES \\
\hline KN Cen & 2009-Jan.-31 & 54862.355 & 80 & 153 & $5130 \pm 68$ & 0.01 & 3.2 & $0.36 \pm 0.28$ & 14 & $0.59 \pm 0.13$ & 3 & UVES \\
\hline MZ Cen & 2008-Apr.-28 & 54584.280 & 800 & 217 & $5520 \pm 55$ & 0.90 & 3.6 & $0.27 \pm 0.14$ & 45 & $0.26 \pm 0.14$ & 4 & UVES \\
\hline OO Cen & 2008-Apr.-29 & 54585.060 & 1500 & 288 & $5950 \pm 65$ & 1.00 & 3.7 & $0.19 \pm 0.12$ & 30 & $0.20 \pm 0.07$ & 4 & UVES \\
\hline TX Cen & 2009-Jan.-31 & 54862.363 & 200 & 152 & $5380 \pm 87$ & 0.60 & 3.8 & $0.43 \pm 0.16$ & 78 & $0.45 \pm 0.20$ & 7 & UVES \\
\hline V339 Cen & 2008-Apr.-28 & 54584.304 & 60 & 183 & $5210 \pm 54$ & 0.40 & 3.2 & $0.06 \pm 0.14$ & 39 & $0.06 \pm 0.03$ & 3 & UVES \\
\hline VW Cen & 2009-Jan.-31 & 54862.359 & 200 & 144 & $5190 \pm 65$ & 0.30 & 4.4 & $0.40 \pm 0.10$ & 43 & $0.44 \pm 0.13$ & 2 & UVES \\
\hline $\mathrm{AO} \mathrm{CMa}$ & 2009-Jan.-08 & 54839.053 & 900 & 120 & $5855 \pm 57$ & 1.30 & 2.6 & $0.00 \pm 0.07$ & 75 & $0.03 \pm 0.11$ & 5 & UVES \\
\hline RW CMa & 2009-Jan.-08 & 54839.138 & 500 & 198 & $6050 \pm 63$ & 1.20 & 3.0 & $-0.07 \pm 0.11$ & 83 & $-0.06 \pm 0.11$ & 5 & UVES \\
\hline SS CMa & 2009-Jan.-08 & 54839.066 & 80 & 93 & $5700 \pm 54$ & 0.60 & 2.6 & $0.06 \pm 0.05$ & 57 & $0.07 \pm 0.10$ & 5 & UVES \\
\hline TV CMa & 2009-Jan.-16 & 54847.246 & 200 & 149 & $5570 \pm 38$ & 1.10 & 3.0 & $0.00 \pm 0.11$ & 89 & $0.01 \pm 0.09$ & 6 & UVES \\
\hline TV CMa & 2007-Nov.-03 & 54407.238 & 2700 & 101 & $5850 \pm 126$ & 1.4 & 2.6 & $0.01 \pm 0.09$ & 128 & $0.06 \pm 0.19$ & 23 & NARVAL \\
\hline TW CMa & 2009-Jan.-08 & 54839.077 & 80 & 63 & $6135 \pm 109$ & 1.20 & 2.5 & $0.04 \pm 0.10$ & 38 & $0.07 \pm 0.30$ & 4 & UVES \\
\hline AA Gem & 2009-Jan.-15 & 54846.149 & 80 & 84 & $5540 \pm 104$ & 0.60 & 3.0 & $-0.08 \pm 0.06$ & 74 & $-0.08 \pm 0.13$ & 5 & UVES \\
\hline AD Gem & 2009-Jan.-15 & 54846.221 & 80 & 109 & $6200 \pm 122$ & 1.60 & 2.2 & $-0.17 \pm 0.14$ & 70 & $-0.13 \pm 0.07$ & 7 & UVES \\
\hline BB Gem & 2009-Jan.-15 & 54846.187 & 500 & 160 & $7000 \pm 253$ & 1.90 & 2.8 & $-0.08 \pm 0.13$ & 70 & $-0.09 \pm 0.04$ & 9 & UVES \\
\hline BW Gem & 2009-Jan.-14 & 54845.122 & 900 & 141 & $5850 \pm 65$ & 1.60 & 2.2 & $-0.22 \pm 0.12$ & 99 & $-0.21 \pm 0.15$ & 6 & UVES \\
\hline DX Gem & 2009-Jan.-15 & 54846.196 & 200 & 121 & $6200 \pm 92$ & 1.60 & 2.8 & $-0.01 \pm 0.11$ & 72 & $-0.01 \pm 0.15$ & 6 & UVES \\
\hline RZ Gem & 2009-Jan.-14 & 54845.094 & 200 & 207 & $7000 \pm 170$ & 1.60 & 3.4 & $-0.13 \pm 0.11$ & 44 & $-0.16 \pm 0.03$ & 5 & UVES \\
\hline BE Mon & 2009-Jan.-15 & 54846.201 & 200 & 136 & $5770 \pm 58$ & 1.50 & 2.9 & $0.05 \pm 0.12$ & 78 & $0.05 \pm 0.13$ & 5 & UVES \\
\hline CV Mon & 2009-Jan.-15 & 54846.182 & 200 & 152 & $5540 \pm 58$ & 0.60 & 2.6 & $0.09 \pm 0.10$ & 52 & $0.11 \pm 0.21$ & 2 & UVES \\
\hline FT Mon & 2009-Jan.-14 & 54845.104 & 1000 & 85 & $5810 \pm 92$ & 1.20 & 2.7 & $-0.13 \pm 0.11$ & 61 & $-0.14 \pm 0.12$ & 8 & UVES \\
\hline SV Mon & 2009-Jan.-14 & 54845.119 & 30 & 143 & $5090 \pm 58$ & 0.10 & 2.5 & $0.10 \pm 0.09$ & 54 & $0.17 \pm 0.15$ & 8 & UVES \\
\hline TW Mon & 2008-Nov.-26 & 54796.347 & 1000 & 144 & $5990 \pm 105$ & 1.30 & 2.7 & $-0.13 \pm 0.08$ & 75 & $-0.13 \pm 0.16$ & 6 & UVES \\
\hline TX Mon & 2008-Nov.-28 & 54798.345 & 200 & 109 & $6180 \pm 62$ & 1.40 & 3.2 & $-0.03 \pm 0.15$ & 76 & $-0.03 \pm 0.05$ & 4 & UVES \\
\hline TY Mon & 2009-Jan.-15 & 54846.139 & 500 & 134 & $6320 \pm 73$ & 1.50 & 2.3 & $0.01 \pm 0.11$ & 85 & $0.03 \pm 0.12$ & 6 & UVES \\
\hline TZ Mon & 2009-Jan.-16 & 54847.237 & 200 & 127 & $5625 \pm 41$ & 1.20 & 2.9 & $-0.02 \pm 0.08$ & 94 & $-0.01 \pm 0.12$ & 6 & UVES \\
\hline V465 Mon & 2009-Jan.-16 & 54847.241 & 200 & 144 & $6250 \pm 56$ & 1.50 & 2.8 & $-0.07 \pm 0.11$ & 107 & $-0.07 \pm 0.10$ & 6 & UVES \\
\hline V495 Mon & 2009-Jan.-15 & 54846.167 & 1000 & 126 & $5590 \pm 57$ & 1.20 & 3.0 & $-0.14 \pm 0.09$ & 73 & $-0.11 \pm 0.10$ & 4 & UVES \\
\hline V508 Mon & 2009-Jan.-16 & 54847.232 & 200 & 135 & $6140 \pm 95$ & 1.70 & 2.7 & $-0.06 \pm 0.15$ & 118 & $-0.03 \pm 0.13$ & 7 & UVES \\
\hline V510 Mon & 2009-Jan.-15 & 54846.153 & 1000 & 125 & $5505 \pm 52$ & 0.90 & 3.4 & $-0.15 \pm 0.11$ & 80 & $-0.17 \pm 0.08$ & 3 & UVES \\
\hline XX Mon & 2008-Nov.-28 & 54798.335 & 500 & 93 & $5590 \pm 47$ & 1.20 & 2.8 & $0.00 \pm 0.09$ & 55 & $0.02 \pm 0.14$ & 2 & UVES \\
\hline GU Nor & 2008-Jul.-20 & 54667.205 & 300 & 258 & $6240 \pm 43$ & 1.40 & 3.2 & $0.08 \pm 0.11$ & 80 & $0.08 \pm 0.07$ & 7 & UVES \\
\hline IQ Nor & 2008-Apr.-28 & 54584.299 & 150 & 265 & $5950 \pm 87$ & 0.70 & 4.0 & $0.19 \pm 0.13$ & 63 & $0.23 \pm 0.09$ & 7 & UVES \\
\hline QZ Nor & 2009-Feb.-01 & 54863.366 & 30 & 100 & $5740 \pm 46$ & 1.10 & 2.8 & $0.18 \pm 0.12$ & 81 & $0.19 \pm 0.12$ & 3 & UVES \\
\hline QZ Nor & 2009-Apr.-02 & 54923.345 & 30 & 100 & $5790 \pm 53$ & 1.00 & 2.8 & $0.20 \pm 0.12$ & 86 & $0.24 \pm 0.09$ & 2 & UVES \\
\hline RS Nor & 2009-Feb.-01 & 54863.361 & 200 & 230 & $6140 \pm 63$ & 1.20 & 3.2 & $0.17 \pm 0.12$ & 82 & $0.19 \pm 0.12$ & 5 & UVES \\
\hline SY Nor & 2008-Aug.-30 & 54708.061 & 150 & 170 & $5550 \pm 64$ & 0.70 & 3.7 & $0.26 \pm 0.14$ & 46 & $0.28 \pm 0.15$ & 5 & UVES \\
\hline SY Nor & 2008-Aug.-31 & 54709.075 & 150 & 205 & $5675 \pm 55$ & 1.00 & 3.6 & $0.20 \pm 0.12$ & 58 & $0.19 \pm 0.13$ & 4 & UVES \\
\hline TW Nor & 2008-Jul.-19 & 54666.127 & 800 & 216 & $5090 \pm 73$ & 0.30 & 3.5 & $0.28 \pm 0.20$ & 69 & $0.27 \pm 0.12$ & 7 & UVES \\
\hline V340 Nor & 2009-Feb.-11 & 54873.376 & 30 & 169 & $5685 \pm 77$ & 0.60 & 3.4 & $0.07 \pm 0.10$ & 47 & $0.08 \pm 0.11$ & 4 & UVES \\
\hline CS Ori & 2009-Jan.-14 & 54845.085 & 500 & 128 & $6390 \pm 162$ & 1.20 & 2.0 & $-0.27 \pm 0.08$ & 68 & $-0.22 \pm 0.09$ & 6 & UVES \\
\hline GQ Ori & 2009-Jan.-14 & 54845.082 & 30 & 100 & $5050 \pm 104$ & 2.80 & 1.2 & $0.19 \pm 0.12$ & 92 & $0.22 \pm 0.12$ & 14 & UVES \\
\hline RS Ori & 2009-Jan.-14 & 54845.100 & 30 & 149 & $6165 \pm 57$ & 1.00 & 2.6 & $0.11 \pm 0.13$ & 71 & $0.11 \pm 0.12$ & 5 & UVES \\
\hline AQ Pup & 2009-Jan.-08 & 54839.075 & 30 & 50 & $5030 \pm 96$ & 0.10 & 4.4 & $-0.07 \pm 0.14$ & 14 & $0.08 \pm 0.05$ & 2 & UVES \\
\hline BC Pup & 2009-Jan.-08 & 54839.147 & 2000 & 81 & $5705 \pm 121$ & 1.40 & 3.2 & $-0.31 \pm 0.09$ & 57 & $-0.32 \pm 0.11$ & 3 & UVES \\
\hline BM Pup & 2009-Jan.-08 & 54839.086 & 200 & 80 & $5526 \pm 84$ & 0.80 & 2.7 & $-0.07 \pm 0.11$ & 61 & $-0.06 \pm 0.11$ & 7 & UVES \\
\hline BN Pup & 2009-Jan.-08 & 54839.109 & 80 & 117 & $5330 \pm 54$ & 0.50 & 2.6 & $0.03 \pm 0.06$ & 69 & $0.05 \pm 0.13$ & 4 & UVES \\
\hline CE Pup & 2010-Mar.-31 & 55286.057 & 6300 & 248 & $5770 \pm 123$ & 0.50 & 4.0 & $-0.04 \pm 0.11$ & 67 & $-0.03 \pm 0.15$ & 3 & FEROS \\
\hline CK Pup & 2009-Jan.-08 & 54839.113 & 2000 & 141 & $6235 \pm 62$ & 1.20 & 2.4 & $-0.15 \pm 0.11$ & 72 & $-0.14 \pm 0.12$ & 4 & UVES \\
\hline CK Pup & 2009-Jan.-08 & 54839.173 & 2000 & 156 & $6200 \pm 108$ & 0.80 & 2.3 & $-0.11 \pm 0.11$ & 78 & $-0.13 \pm 0.11$ & 11 & UVES \\
\hline HW Pup & 2008-Nov.-22 & 54792.249 & 900 & 123 & $5200 \pm 61$ & 0.40 & 2.6 & $-0.24 \pm 0.10$ & 70 & $-0.16 \pm 0.17$ & 3 & UVES \\
\hline LS Pup & 2009-Jan.-08 & 54839.081 & 200 & 97 & $5235 \pm 115$ & 0.40 & 3.6 & $-0.12 \pm 0.11$ & 18 & $-0.13 \pm 0.00$ & 1 & UVES \\
\hline VW Pup & 2009-Jan.-01 & 54832.331 & 500 & 142 & $5600 \pm 78$ & 1.20 & 3.1 & $-0.14 \pm 0.07$ & 50 & $-0.16 \pm 0.13$ & 4 & UVES \\
\hline VZ Pup & 2009-Jan.-08 & 54839.096 & 80 & 123 & $6480 \pm 65$ & 1.00 & 3.1 & $0.01 \pm 0.05$ & 27 & $-0.05 \pm 0.06$ & 2 & UVES \\
\hline WW Pup & 2009-Jan.-08 & 54839.091 & 200 & 119 & $5500 \pm 104$ & 1.20 & 3.2 & $0.13 \pm 0.16$ & 18 & $0.13 \pm 0.00$ & 1 & UVES \\
\hline WY Pup & 2009-Jan.-08 & 54839.100 & 200 & 102 & $6020 \pm 76$ & 1.60 & 3.2 & $-0.10 \pm 0.10$ & 49 & $-0.09 \pm 0.12$ & 6 & UVES \\
\hline WZ Pup & 2009-Jan.-08 & 54839.104 & 200 & 113 & $5660 \pm 41$ & 1.00 & 2.7 & $-0.07 \pm 0.07$ & 72 & $-0.07 \pm 0.09$ & 7 & UVES \\
\hline X Pup & 2009-Jan.-08 & 54839.070 & 30 & 69 & $5070 \pm 115$ & 0.10 & 4.8 & $0.00 \pm 0.09$ & 15 & $0.09 \pm 0.16$ & 2 & UVES \\
\hline KQ Sco & 2012-Jul.-31 & 56139.021 & 100 & 87 & $5030 \pm 40$ & 0.10 & 4.8 & $0.26 \pm 0.15$ & 32 & $0.00 \pm 0.00$ & 0 & UVES \\
\hline KQ Sco & 2009-Feb.-11 & 54873.379 & 80 & 150 & $4905 \pm 80$ & 0.10 & 4.6 & $0.51 \pm 0.08$ & 51 & $0.63 \pm 0.27$ & 4 & UVES \\
\hline KQ Sco & 2012-Aug.-13 & 56152.097 & 100 & 100 & $4915 \pm 53$ & 0.30 & 3.6 & $0.30 \pm 0.21$ & 16 & $0.35 \pm 0.00$ & 1 & UVES \\
\hline KQ Sco & 2012-Aug.-24 & 56163.004 & 100 & 85 & $4840 \pm 62$ & 0.10 & 5.2 & $0.22 \pm 0.27$ & 20 & $0.63 \pm 0.00$ & 1 & UVES \\
\hline KQ Sco & 2012-Aug.-27 & 56166.004 & 100 & 97 & $4930 \pm 53$ & 0.20 & 4.8 & $0.21 \pm 0.28$ & 15 & $0.39 \pm 0.00$ & 1 & UVES \\
\hline RY Sco & 2012-Aug.-01 & 56140.187 & 20 & 116 & $5545 \pm 40$ & 0.60 & 3.6 & $0.02 \pm 0.14$ & 74 & $0.06 \pm 0.01$ & 2 & UVES \\
\hline RY Sco & 2008-Маy-13 & 54599.412 & 20 & 136 & $5290 \pm 40$ & 0.40 & 3.7 & $0.06 \pm 0.09$ & 34 & $0.06 \pm 0.02$ & 5 & UVES \\
\hline RY Sco & 2012-Aug.-13 & 56152.143 & 20 & 116 & $6200 \pm 54$ & 0.80 & 5.2 & $0.01 \pm 0.15$ & 75 & $0.01 \pm 0.03$ & 3 & UVES \\
\hline RY Sco & 2012-Aug.-23 & 56162.170 & 20 & 108 & $5380 \pm 38$ & 0.40 & 3.8 & $0.00 \pm 0.14$ & 66 & $-0.03 \pm 0.05$ & 2 & UVES \\
\hline RY Sco & 2012-Aug.-28 & 56167.085 & 20 & 111 & $5330 \pm 31$ & 0.70 & 4.8 & $-0.05 \pm 0.12$ & 45 & $-0.03 \pm 0.11$ & 2 & UVES \\
\hline
\end{tabular}

Notes. From left to right the columns give the Cepheid name, the date of acquisition and the modified Julian Day of the spectrum, the exposure time, maximum $\mathrm{S} / \mathrm{N}$ of the spectrum, the estimated intrinsic parameters $\left(T_{\text {eff }}, \log g, v_{\mathrm{t}}\right)$. Columns 9 and 10 list the Fe I abundance and its standard deviation together with the number of Fe I lines measured. Columns 11 and 12 list the Fe II abundance and its standard deviation together with the number of Fe II lines measured. The last column gives the name of the adopted spectrograph. 
Table 1. continued.

\begin{tabular}{|c|c|c|c|c|c|c|c|c|c|c|c|c|}
\hline Name & Date & MJD & $\begin{array}{r}E T \\
(\mathrm{~s})\end{array}$ & $S / N$ & $\begin{array}{c}T_{\text {eff }} \\
\mathrm{K}\end{array}$ & $\log g$ & $\begin{array}{c}v_{\mathrm{t}} \\
\left(\mathrm{km} \mathrm{s}^{-1}\right)\end{array}$ & Fe I & $N$ & Fe II & $N$ & Spect. \\
\hline V470 Sco & 2008-Aug.-30 & 54708.073 & 800 & 380 & $5730 \pm 33$ & 0.80 & 4.4 & $0.16 \pm 0.10$ & 66 & $0.16 \pm 0.08$ & 4 & UVES \\
\hline V500 Sco & 2012-Aug.-01 & 56140.191 & 46 & 126 & $5980 \pm 27$ & 1.10 & 4.6 & $-0.09 \pm 0.16$ & 86 & $0.00 \pm 0.05$ & 3 & UVES \\
\hline V500 Sco & 2012-Aug.-13 & 56152.092 & 45 & 99 & $5330 \pm 26$ & 0.60 & 3.2 & $-0.04 \pm 0.12$ & 67 & $0.10 \pm 0.20$ & 3 & UVES \\
\hline V500 Sco & 2012-Aug.-23 & 56162.998 & 45 & 101 & $5280 \pm 31$ & 0.40 & 3.8 & $-0.02 \pm 0.17$ & 53 & $-0.04 \pm 0.17$ & 3 & UVES \\
\hline V500 Sco & 2012-Aug.-28 & 56167.077 & 45 & 152 & $6020 \pm 35$ & 1.10 & 4.0 & $-0.11 \pm 0.16$ & 97 & $-0.11 \pm 0.08$ & 5 & UVES \\
\hline EV Sct & 2008-Aug.-30 & 54708.086 & 300 & 224 & $6025 \pm 65$ & 1.50 & 2.5 & $0.10 \pm 0.15$ & 57 & $0.09 \pm 0.08$ & 3 & UVES \\
\hline RU Sct & 2009-Mar.-16 & 54906.414 & 120 & 205 & $5190 \pm 35$ & 0.10 & 2.9 & $0.15 \pm 0.12$ & 95 & $0.16 \pm 0.05$ & 7 & UVES \\
\hline RU Sct & 2009-Apr.-02 & 54923.375 & 80 & 216 & $5495 \pm 48$ & 0.30 & 3.0 & $0.09 \pm 0.10$ & 45 & $0.09 \pm 0.09$ & 5 & UVES \\
\hline RU Sct & 2007-Oct.-31 & 54404.777 & 2000 & 208 & $6450 \pm 142$ & 0.7 & 3.7 & $-0.07 \pm 0.10$ & 74 & $-0.06 \pm 0.14$ & 15 & NARVAL \\
\hline UZ Sct & 2012-Jul.-29 & 56137.160 & 360 & 105 & $4850 \pm 55$ & 0.60 & 4.0 & $0.26 \pm 0.20$ & 17 & $0.29 \pm 0.16$ & 4 & UVES \\
\hline UZ Sct & 2009-Mar.-16 & 54906.400 & 700 & 273 & $5470 \pm 76$ & 0.80 & 2.8 & $0.36 \pm 0.19$ & 34 & $0.36 \pm 0.12$ & 5 & UVES \\
\hline UZ Sct & 2009-Apr.-02 & 54923.366 & 500 & 249 & $5060 \pm 76$ & 0.20 & 2.9 & $0.45 \pm 0.08$ & 63 & $0.43 \pm 0.15$ & 7 & UVES \\
\hline UZ Sct & 2012-Aug.-13 & 56152.064 & 360 & 70 & $4850 \pm 49$ & 0.80 & 4.4 & $0.25 \pm 0.28$ & 8 & $0.00 \pm 0.00$ & 0 & UVES \\
\hline UZ Sct & 2012-Aug.-21 & 56160.167 & 360 & 117 & $5370 \pm 76$ & 0.60 & 3.2 & $0.34 \pm 0.23$ & 47 & $0.37 \pm 0.11$ & 2 & UVES \\
\hline UZ Sct & 2012-Sep.-05 & 56175.049 & 360 & 116 & $5320 \pm 85$ & 0.60 & 3.2 & $0.31 \pm 0.21$ & 36 & $0.34 \pm 0.00$ & 2 & UVES \\
\hline V367 Sct & 2012-Jul.-29 & 56137.147 & 360 & 98 & $5670 \pm 56$ & 1.00 & 3.8 & $0.08 \pm 0.18$ & 72 & $0.14 \pm 0.08$ & 3 & UVES \\
\hline V367 Sct & 2008-Aug.-31 & 54709.128 & 800 & 255 & $5740 \pm 51$ & 1.30 & 3.4 & $-0.05 \pm 0.13$ & 56 & $-0.04 \pm 0.04$ & 4 & UVES \\
\hline V367 Sct & 2012-Sep.-05 & 56175.105 & 360 & 82 & $5930 \pm 64$ & 1.20 & 4.2 & $0.15 \pm 0.15$ & 50 & $0.14 \pm 0.07$ & 3 & UVES \\
\hline V367 Sct & 2012-Sep.-14 & 56184.000 & 360 & 123 & $6200 \pm 91$ & 1.00 & 3.4 & $0.04 \pm 0.15$ & 84 & $0.03 \pm 0.08$ & 4 & UVES \\
\hline X Sct & 2007-Nov.-01 & 54405.762 & 2400 & 128 & $6300 \pm 138$ & 1.2 & 2.7 & $0.05 \pm 0.16$ & 82 & $0.16 \pm 0.26$ & 23 & NARVAL \\
\hline X Sct & 2008-Aug.-31 & 54709.122 & 300 & 231 & $5830 \pm 83$ & 1.10 & 2.5 & $0.11 \pm 0.12$ & 72 & $0.14 \pm 0.15$ & 9 & UVES \\
\hline Z Sct & 2012-Jul.-29 & 56137.123 & 100 & 111 & $5130 \pm 70$ & 0.20 & 4.6 & $0.10 \pm 0.16$ & 20 & $0.00 \pm 0.00$ & 0 & UVES \\
\hline Z Sct & 2008-Jul.-31 & 54678.090 & 150 & 185 & $5005 \pm 78$ & 0.05 & 3.8 & $0.18 \pm 0.14$ & 41 & $0.18 \pm 0.12$ & 3 & UVES \\
\hline Z Sct & 2012-Aug.-13 & 56152.073 & 101 & 96 & $5650 \pm 61$ & 0.90 & 5.2 & $0.09 \pm 0.14$ & 49 & $0.11 \pm 0.02$ & 2 & UVES \\
\hline Z Sct & 2012-Aug.-20 & 56159.186 & 100 & 103 & $5160 \pm 64$ & 0.20 & 3.4 & $0.21 \pm 0.22$ & 45 & $0.27 \pm 0.08$ & 2 & UVES \\
\hline Z Sct & 2012-Sep.-05 & 56175.038 & 100 & 89 & $5005 \pm 68$ & 0.40 & 4.6 & $0.00 \pm 0.30$ & 25 & $0.00 \pm 0.00$ & 0 & UVES \\
\hline AA Ser & 2008-Aug.-30 & 54708.040 & 1500 & 231 & $4830 \pm 80$ & 0.20 & 3.2 & $0.38 \pm 0.20$ & 24 & $0.37 \pm 0.00$ & 1 & UVES \\
\hline CR Ser & 2008-Aug.-31 & 54709.116 & 300 & 122 & $5500 \pm 48$ & 0.80 & 3.3 & $0.13 \pm 0.11$ & 53 & $0.11 \pm 0.11$ & 5 & UVES \\
\hline AV Sgr & 2012-Jul.-28 & 56136.169 & 361 & 87 & $5070 \pm 56$ & 0.20 & 3.6 & $0.37 \pm 0.21$ & 29 & $0.43 \pm 0.21$ & 2 & UVES \\
\hline AV Sgr & 2012-Jul.-28 & 56136.192 & 1440 & 173 & $5080 \pm 51$ & 0.10 & 3.4 & $0.43 \pm 0.23$ & 31 & $0.45 \pm 0.20$ & 2 & UVES \\
\hline AV Sgr & 2009-Apr.-02 & 54923.348 & 500 & 233 & $5060 \pm 50$ & 0.10 & 4.5 & $0.35 \pm 0.30$ & 16 & $0.61 \pm 0.20$ & 2 & UVES \\
\hline AV Sgr & 2012-Aug.-13 & 56152.082 & 361 & 62 & $5020 \pm 58$ & 0.30 & 3.6 & $0.29 \pm 0.23$ & 24 & $0.58 \pm 0.25$ & 2 & UVES \\
\hline AV Sgr & 2012-Aug.-29 & 56168.049 & 360 & 75 & $5020 \pm 49$ & 0.40 & 3.6 & $0.30 \pm 0.22$ & 19 & $0.35 \pm 0.00$ & 1 & UVES \\
\hline AY Sgr & 2008-Мay-13 & 54599.398 & 300 & 195 & $5760 \pm 52$ & 0.90 & 3.0 & $0.11 \pm 0.14$ & 58 & $0.11 \pm 0.07$ & 5 & UVES \\
\hline V1954 Sgr & 2008-Мay-13 & 54599.389 & 300 & 158 & $6120 \pm 33$ & 1.10 & 3.5 & $0.25 \pm 0.13$ & 61 & $0.22 \pm 0.17$ & 4 & UVES \\
\hline V773 Sgr & 2008-Jul.-22 & 54669.207 & 1500 & 264 & $6195 \pm 39$ & 1.00 & 3.4 & $0.11 \pm 0.07$ & 58 & $0.10 \pm 0.11$ & 8 & UVES \\
\hline VY Sgr & 2012-Aug.-21 & 56160.179 & 360 & 76 & $4900 \pm 46$ & 0.20 & 4.6 & $0.27 \pm 0.25$ & 14 & $0.56 \pm 0.00$ & 1 & UVES \\
\hline VY Sgr & 2009-Apr.-02 & 54923.356 & 500 & 231 & $5100 \pm 84$ & 0.40 & 2.6 & $0.37 \pm 0.19$ & 30 & $0.49 \pm 0.21$ & 6 & UVES \\
\hline VY Sgr & 2012-Aug.-23 & 56162.162 & 360 & 83 & $5230 \pm 91$ & 0.10 & 5.2 & $0.32 \pm 0.27$ & 17 & $0.30 \pm 0.00$ & 1 & UVES \\
\hline VY Sgr & 2012-Aug.-29 & 56168.062 & 360 & 81 & $5470 \pm 77$ & 0.60 & 3.4 & $0.32 \pm 0.19$ & 51 & $0.31 \pm 0.05$ & 2 & UVES \\
\hline WZ Sgr & 2012-Jul.-24 & 56132.190 & 20 & 134 & $5990 \pm 72$ & 0.90 & 5.6 & $0.19 \pm 0.11$ & 56 & $0.18 \pm 0.11$ & 2 & UVES \\
\hline WZ Sgr & 2008-May-13 & 54599.395 & 20 & 101 & $5115 \pm 65$ & 0.05 & 4.6 & $0.36 \pm 0.10$ & 42 & $0.32 \pm 0.14$ & 2 & UVES \\
\hline WZ Sgr & 2012-Jul.-28 & 56136.213 & 80 & 267 & $5500 \pm 70$ & 0.50 & 3.5 & $0.24 \pm 0.16$ & 48 & $0.24 \pm 0.01$ & 2 & UVES \\
\hline WZ Sgr & 2012-Aug.-13 & 56152.044 & 20 & 87 & $5200 \pm 56$ & 0.20 & 5.0 & $0.25 \pm 0.26$ & 28 & $0.29 \pm 0.14$ & 2 & UVES \\
\hline WZ Sgr & 2012-Aug.-20 & 56159.125 & 20 & 129 & $5335 \pm 58$ & 0.30 & 3.2 & $0.34 \pm 0.18$ & 44 & $0.37 \pm 0.06$ & 2 & UVES \\
\hline XX Sgr & 2012-May-07 & 56054.234 & 45 & 121 & $5840 \pm 49$ & 1.20 & 3.4 & $-0.02 \pm 0.14$ & 100 & $-0.01 \pm 0.13$ & 5 & UVES \\
\hline XX Sgr & 2008-May-13 & 54599.404 & 60 & 184 & $6340 \pm 84$ & 1.40 & 3.6 & $-0.03 \pm 0.12$ & 59 & $-0.09 \pm 0.08$ & 4 & UVES \\
\hline XX Sgr & 2012-Jul.-28 & 56136.223 & 180 & 257 & $6200 \pm 40$ & 1.00 & 3.4 & $-0.05 \pm 0.12$ & 101 & $-0.05 \pm 0.05$ & 6 & UVES \\
\hline XX Sgr & 2012-Aug.-13 & 56152.047 & 46 & 84 & $5520 \pm 48$ & 0.80 & 3.0 & $0.05 \pm 0.08$ & 43 & $0.05 \pm 0.03$ & 3 & UVES \\
\hline XX Sgr & 2012-Aug.-20 & 56159.128 & 45 & 95 & $5525 \pm 55$ & 0.90 & 3.6 & $-0.01 \pm 0.18$ & 64 & $-0.03 \pm 0.15$ & 4 & UVES \\
\hline EZ Vel & 2008-Oct.-20 & 54759.348 & 1000 & 89 & $4735 \pm 75$ & 0.10 & 3.9 & $-0.17 \pm 0.15$ & 23 & $-0.10 \pm 0.00$ & 1 & UVES \\
\hline
\end{tabular}


K. Genovali et al.: The fine structure of the metallicity gradient

Table 3. Mean NIR magnitudes, mean distances and mean iron abundances for the current sample of classical Cepheids.

\begin{tabular}{|c|c|c|c|c|c|c|c|c|c|c|c|c|}
\hline Name & Type & $\begin{array}{l}\log (P) \\
\text { [days] }\end{array}$ & $\begin{array}{c}\langle J\rangle \\
\mathrm{mag}\end{array}$ & $\begin{array}{l}\langle H\rangle \\
\mathrm{mag}\end{array}$ & $\begin{array}{c}\langle K\rangle \\
\mathrm{mag}\end{array}$ & {$[\mathrm{Fe} / \mathrm{H}]_{\text {lit }}$} & {$[\mathrm{Fe} / \mathrm{H}]$} & $N_{\mathrm{S}}$ & $\begin{array}{c}\mu^{d} \\
\mathrm{mag}\end{array}$ & $\begin{array}{l}R_{\mathrm{G}}^{\mathrm{e}} \\
(\mathrm{pc})\end{array}$ & Notes & Ref. ${ }^{f}$ \\
\hline V340 Ara & DCEP & 1.3183 & 7.302 & 6.754 & 6.534 & 0.40 & $0.33 \pm 0.09$ & 6 & $12.99 \pm 0.05$ & $4657 \pm 427$ & $\mathrm{~b}^{\dagger}$ & PED \\
\hline $\mathrm{AO} \mathrm{Cma}$ & DCEP & 0.7646 & 9.154 & 8.595 & 8.366 & -0.04 & $0.01 \pm 0.06$ & 1 & $12.95 \pm 0.05$ & $10430 \pm 433$ & $\mathrm{~b}^{\dagger}$ & LEM \\
\hline TW Cma & DCEP & 0.8448 & 7.567 & 7.178 & 7.008 & -0.51 & $0.04 \pm 0.09$ & 1 & $12.02 \pm 0.05$ & $9788 \pm 445$ & $\mathrm{a}$ & LEM \\
\hline AD Gem & DCEP & 0.5784 & 8.448 & 8.151 & 8.027 & -0.19 & $-0.14 \pm 0.06$ & 1 & $12.24 \pm 0.05$ & $10662 \pm 455$ & $\mathrm{a}$ & LEM \\
\hline BB Gem & DCEP & 0.3633 & 9.696 & 9.335 & 9.215 & -0.10 & $-0.09 \pm 0.04$ & 1 & $12.66 \pm 0.07$ & $11199 \pm 460$ & $\mathrm{c}$ & LIII \\
\hline DX Gem & DCEPS & 0.4966 & 8.759 & 8.336 & 8.207 & -0.04 & $-0.01 \pm 0.09$ & 1 & $12.78 \pm 0.09$ & $11407 \pm 473$ & $\mathrm{c}^{*}$ & LIII \\
\hline RZ Gem & DCEP' & 0.7427 & 7.600 & 7.165 & 6.950 & -0.44 & $-0.16 \pm 0.03$ & 1 & $11.56 \pm 0.05$ & $9973 \pm 454$ & $\mathrm{a}$ & LEM \\
\hline BE Mon & DCEP & 0.4322 & 8.256 & 7.854 & 7.676 & -0.07 & $0.05 \pm 0.09$ & 1 & $11.28 \pm 0.05$ & $9609 \pm 452$ & $\mathrm{a}$ & LEM \\
\hline CV Mon & DCEP & 0.7307 & 7.314 & 6.781 & 6.529 & -0.10 & $0.09 \pm 0.09$ & 1 & $11.00 \pm 0.05$ & $9362 \pm 452$ & a & LEM \\
\hline TW Mon & CEP & 0.8511 & 9.709 & 9.137 & 8.904 & -0.15 & $-0.13 \pm 0.07$ & 1 & $13.76 \pm 0.05$ & $13059 \pm 457$ & $\mathrm{~b}^{\dagger}$ & LEM \\
\hline TX Mon & DCEP & 0.9396 & 8.569 & 8.114 & 7.922 & -0.12 & $-0.03 \pm 0.05$ & 1 & $13.19 \pm 0.05$ & $11790 \pm 452$ & $\mathrm{a}^{\dagger}$ & LEM \\
\hline V465 Mon & DCEP & 0.4335 & 8.751 & 8.440 & 8.315 & 0.02 & $-0.07 \pm 0.07$ & 1 & $12.75 \pm 0.05$ & $11037 \pm 450$ & a & LIII \\
\hline V495 Mon & DCEP & 0.6124 & 9.827 & 9.351 & 9.185 & -0.17 & $-0.13 \pm 0.07$ & 1 & $13.35 \pm 0.05$ & $12098 \pm 453$ & $\mathrm{a}^{\dagger}$ & LEM \\
\hline V508 Mon & DCEP & 0.6163 & 8.627 & 8.277 & 8.136 & -0.25 & $-0.04 \pm 0.10$ & 1 & $12.42 \pm 0.05$ & $10714 \pm 452$ & $\mathrm{a}$ & LEM \\
\hline V510 Mon & DCEP & 0.8637 & 9.507 & 8.898 & 8.648 & -0.12 & $-0.16 \pm 0.06$ & 1 & $13.51 \pm 0.05$ & $12550 \pm 456$ & $\mathrm{~b}^{\dagger}$ & LEM \\
\hline XX Mon & DCEP & 0.7369 & 9.402 & 8.903 & 8.699 & -0.18 & $0.01 \pm 0.08$ & 1 & $13.25 \pm 0.05$ & $11854 \pm 451$ & $\mathrm{~b}^{\dagger}$ & LEM \\
\hline QZ Nor & DCEPS & 0.5782 & 7.085 & 6.748 & 6.614 & 0.19 & $0.21 \pm 0.06$ & 2 & $11.53 \pm 0.05$ & $6283 \pm 447$ & b & G13 \\
\hline SY Nor & DCEP & 1.1019 & 6.638 & 6.091 & 5.864 & 0.34 & $0.23 \pm 0.07$ & 2 & $11.59 \pm 0.05$ & $6286 \pm 446$ & $\mathrm{~b}^{\dagger}$ & LIII \\
\hline CS Ori & DCEP & 0.5899 & 9.331 & 8.954 & 8.790 & -0.19 & $-0.25 \pm 0.06$ & 1 & $12.96 \pm 0.05$ & $11701 \pm 458$ & $\mathrm{a}$ & LEM \\
\hline AQ Pup & DCEP & 1.4786 & 6.033 & 5.495 & 5.283 & -0.26 & $0.06 \pm 0.05$ & 1 & $12.29 \pm 0.05$ & $9472 \pm 436$ & $\mathrm{~b}$ & LEM \\
\hline BC Pup & DCEP & 0.5495 & 11.015 & 10.475 & 10.247 & -0.23 & $-0.31 \pm 0.07$ & 1 & $14.12 \pm 0.05$ & $12763 \pm 426$ & $\mathrm{~b}^{\dagger}$ & LII \\
\hline BN Pup & DCEP & 1.1359 & 7.563 & 7.090 & 6.910 & -0.03 & $0.03 \pm 0.05$ & 1 & $12.83 \pm 0.05$ & $9930 \pm 428$ & $\mathrm{~b}$ & LEM \\
\hline CE Pup & DCEP & 1.6949 & 8.402 & 7.816 & 7.581 & -0.04 & $-0.04 \pm 0.09$ & 1 & $15.27 \pm 0.07$ & $14958 \pm 422$ & $\mathrm{c}$ & LII \\
\hline CK Pup & CEP & 0.8703 & 10.273 & 9.703 & 9.456 & -0.12 & $-0.13 \pm 0.06$ & 2 & $14.37 \pm 0.05$ & $13357 \pm 423$ & $\mathrm{~b}^{\dagger}$ & G13 \\
\hline HW Pup & DCEP & 1.1289 & 9.468 & 8.914 & 8.716 & -0.28 & $-0.22 \pm 0.09$ & 1 & $14.55 \pm 0.07$ & $13554 \pm 436$ & $\mathrm{c}$ & LEM \\
\hline LS Pup & CEP & 1.1506 & 8.030 & 7.531 & 7.341 & -0.15 & $-0.12 \pm 0.11$ & 1 & $13.29 \pm 0.05$ & $10610 \pm 423$ & $\mathrm{~b}$ & ROM \\
\hline VW Pup & DCEP & 0.6320 & 9.008 & 8.514 & 8.360 & -0.19 & $-0.14 \pm 0.06$ & 1 & $12.58 \pm 0.07$ & $10175 \pm 443$ & $\mathrm{c}$ & LII \\
\hline VZ Pup & DCEP & 1.3649 & 7.309 & 6.842 & 6.657 & -0.37 & $-0.01 \pm 0.04$ & 1 & $13.35 \pm 0.05$ & $10867 \pm 425$ & b & LEM \\
\hline WW Pup & DCEP & 0.7417 & 8.653 & 8.274 & 8.131 & -0.18 & $0.13 \pm 0.16$ & 1 & $12.82 \pm 0.05$ & $10382 \pm 436$ & $\mathrm{~b}^{\dagger}$ & LII \\
\hline X Pup & DCEP & 1.4143 & 6.117 & 5.614 & 5.418 & 0.05 & $0.02 \pm 0.08$ & 1 & $12.24 \pm 0.05$ & $9788 \pm 441$ & b & ROM \\
\hline KQ Sco & DCEP & 1.4577 & 5.945 & 5.229 & 4.924 & 0.22 & $0.52 \pm 0.08$ & 5 & $11.67 \pm 0.05$ & $5948 \pm 451$ & $\mathrm{~b}$ & ROM \\
\hline RY Sco & DCEP & 1.3078 & 4.930 & 4.379 & 4.134 & 0.09 & $0.01 \pm 0.06$ & 5 & $10.54 \pm 0.05$ & $6663 \pm 453$ & $\mathrm{~b}$ & LII \\
\hline V500 Sco & DCEP & 0.9693 & 6.041 & 5.532 & 5.330 & 0.01 & $-0.07 \pm 0.08$ & 4 & $10.65 \pm 0.05$ & $6590 \pm 453$ & $\mathrm{~b}$ & LII \\
\hline RU Sct & DCEP & 1.2945 & 5.891 & 5.287 & 5.034 & 0.11 & $0.14 \pm 0.04$ & 2 & $11.35 \pm 0.05$ & $6361 \pm 449$ & $\mathrm{a}$ & LIII \\
\hline UZ Sct & DCEP & 1.1686 & 7.405 & 6.749 & 6.461 & 0.35 & $0.33 \pm 0.08$ & 6 & $12.29 \pm 0.05$ & $5309 \pm 448$ & $a^{\dagger}$ & PED \\
\hline V367 Sct & CEP(B) & 0.7989 & 7.605 & 6.955 & 6.651 & -0.01 & $0.05 \pm 0.08$ & 4 & $11.23 \pm 0.05$ & $6332 \pm 451$ & $\mathrm{~b}$ & LII \\
\hline Z Sct & DCEP & 1.1106 & 6.949 & 6.477 & 6.263 & 0.33 & $0.12 \pm 0.09$ & 5 & $12.08 \pm 0.05$ & $5733 \pm 445$ & $\mathrm{a}$ & LIII \\
\hline AV Sgr & DCEP & 1.1879 & 6.878 & 6.089 & 5.730 & 0.27 & $0.35 \pm 0.17$ & 5 & $11.49 \pm 0.05$ & $5980 \pm 454$ & $\mathrm{~b}^{\dagger}$ & PED \\
\hline VY Sgr & DCEP & 1.1322 & 7.156 & 6.385 & 6.042 & 0.35 & $0.33 \pm 0.12$ & 5 & $11.63 \pm 0.05$ & $5862 \pm 453$ & $\mathrm{~b}^{\dagger}$ & PED \\
\hline WZ Sgr & DCEP & 1.3394 & 5.255 & 4.752 & 4.536 & 0.19 & $0.28 \pm 0.08$ & 5 & $11.10 \pm 0.05$ & $6326 \pm 453$ & $\mathrm{a}$ & LII \\
\hline XX Sgr & CEP & 0.8078 & 6.436 & 5.967 & 5.747 & 0.10 & $-0.01 \pm 0.06$ & 5 & $10.55 \pm 0.07$ & $6706 \pm 453$ & $\mathrm{c}$ & LII \\
\hline EZ Vel & DCEP & 1.5383 & 8.884 & 8.203 & 7.914 & -0.01 & $-0.17 \pm 0.15$ & 1 & $14.97 \pm 0.07$ & $12119 \pm 358$ & $\mathrm{c}$ & LEM \\
\hline
\end{tabular}

Notes. From left to right the columns give the target name; the variable type (from GCVS); the logarithmic period; the mean magnitudes in $J$, $H$, and $K$ bands. Columns 7 and 8 give the iron abundance available in the literature rescaled to our solar abundance, and the current iron abundance. Column 9 gives the number of spectra used to estimate the mean iron abundance. Columns 10 and 11 list the true distance modulus and the Galactocentric distance. The last two columns give the notes on the NIR photometry and distances for individual objects and the references. a) Mean magnitudes provided by Monson \& Pierce (2011) transformed into the 2MASS photometric system using the zero-points given in their Table 1. b) Mean magnitudes provided by Laney \& Stobie (1992) and by Laney (priv. comm.) transformed into the 2MASS photometric system using the zero-points provided by Koen et al. (2007). The objects marked with a ${ }^{\dagger}$ (Laney, priv. comm.) do not have a complete coverage of the light-curve (the number of phase points ranges from 4 to 14). c) Mean magnitudes based on single-epoch measurements from the 2MASS catalog and the NIR template light curves provided by Soszyński et al. (2005). An asterisk indicates the use of single-epoch photometry in the distance determination (see Sect. 3 for details). For these stars the magnitudes listed in Cols. 4-6 are the single-epoch measurements retrieved from the 2MASS catalog. d) The weighted mean of the three true distance moduli. The errors account for uncertainties affecting the mean magnitudes and for the intrinsic dispersion of the adopted NIR PW relations. e) The weighted mean Galactocentric distances were estimated assuming $R_{\mathrm{G}}=7.94 \pm 0.37 \pm 0.26 \mathrm{kpc}$ (Groenewegen et al. 2008). The errors account for uncertainties affecting both the solar Galactocentric distance and the heliocentric distances. f) References for the iron estimate given in Col. 7. The priority was given, in the following order, to evalutions from our group (G13: Genovali et al. 2013; PED: Pedicelli et al. (2010); LEM: Lemasle et al. (2007, 2008); ROM: Romaniello et al. (2008)) and from the literature: LII: Luck et al. (2011); LIII: Luck \& Lambert (2011). 
Table 4. Galactic Cepheids for which the iron abundance was available in the literature.

\begin{tabular}{|c|c|c|c|c|c|c|c|c|c|c|c|}
\hline Name & Type & $\begin{array}{l}\log (P) \\
\text { [days] }\end{array}$ & $\begin{array}{c}\langle J\rangle \\
\mathrm{mag}\end{array}$ & $\begin{array}{l}\langle H\rangle \\
\mathrm{mag}\end{array}$ & $\begin{array}{c}\langle K\rangle \\
\mathrm{mag}\end{array}$ & {$[\mathrm{Fe} / \mathrm{H}]_{\text {lit }}$} & {$[\mathrm{Fe} / \mathrm{H}]$} & $\begin{array}{c}\mu^{d} \\
\mathrm{mag}\end{array}$ & $\begin{array}{l}R_{\mathrm{G}}^{\mathrm{e}} \\
(\mathrm{pc})\end{array}$ & Notes & Ref. $^{f}$ \\
\hline T Ant & DCEP & 0.7707 & 7.914 & 7.602 & 7.494 & -0.20 & -0.23 & $12.35 \pm 0.05$ & $8706 \pm 427$ & $\mathrm{~b}$ & LIII \\
\hline BC Aql & CEP & 0.4632 & 11.634 & 11.283 & 11.180 & -0.28 & -0.31 & $14.97 \pm 0.07$ & $4578 \pm 190$ & $\mathrm{c}$ & LIII \\
\hline EV Aql & CEP & 1.5885 & 8.990 & 8.369 & 8.162 & 0.06 & 0.03 & $15.48 \pm 0.07$ & $9249 \pm 287$ & $\mathrm{c}$ & LIII \\
\hline FF Aql & DCEPS & 0.6504 & 4.030 & 3.741 & 3.525 & 0.04 & 0.10 & $8.71 \pm 0.37$ & $7592 \pm 455$ & $\mathrm{c}^{*}$ & LII \\
\hline FM Aql & DCEP & 0.7863 & 5.657 & 5.210 & 5.007 & 0.24 & 0.21 & $9.76 \pm 0.05$ & $7326 \pm 450$ & a & LIII \\
\hline FN Aql & DCEPS & 0.9769 & 5.940 & 5.485 & 5.291 & -0.06 & -0.09 & $11.47 \pm 0.05$ & $6520 \pm 445$ & $\mathrm{a}$ & LIII \\
\hline KL Aql & DCEP & 0.7859 & 8.488 & 8.114 & 7.979 & 0.33 & 0.30 & $12.82 \pm 0.07$ & $6573 \pm 403$ & $\mathrm{c}$ & LIII \\
\hline SZ Aql & DCEP & 1.2340 & 5.836 & 5.337 & 5.121 & 0.18 & 0.24 & $11.33 \pm 0.05$ & $6527 \pm 447$ & $\mathrm{a}$ & LII \\
\hline TT Aql & DCEP & 1.1384 & 4.634 & 4.174 & 3.998 & 0.22 & 0.19 & $9.94 \pm 0.05$ & $7176 \pm 451$ & $\mathrm{a}$ & LIII \\
\hline $\mathrm{U}$ Aql & DCEP & 0.8466 & 4.400 & 4.002 & 3.844 & 0.17 & 0.14 & $8.86 \pm 0.05$ & $7448 \pm 451$ & $\mathrm{~b}$ & LIII \\
\hline V1162 Aql & DCEPS & 0.7305 & 6.133 & 5.813 & 5.663 & 0.01 & 0.07 & $11.11 \pm 0.05$ & $6609 \pm 450$ & $\mathrm{a}$ & LII \\
\hline V1344 Aql & DCEP & 0.8738 & 5.177 & 4.732 & 4.536 & 0.15 & 0.12 & $9.59 \pm 0.05$ & $7317 \pm 451$ & $\mathrm{a}$ & LIII \\
\hline V1359 Aql & DCEPS: & 0.5719 & 6.505 & 5.824 & 5.612 & 0.28 & 0.25 & $10.20 \pm 0.07$ & $7148 \pm 450$ & $\mathrm{c}^{*}$ & LIII \\
\hline V336 Aql & DCEP & 0.8635 & 7.149 & 6.688 & 6.484 & 0.18 & 0.15 & $11.48 \pm 0.05$ & $6399 \pm 446$ & $\mathrm{a}$ & LIII \\
\hline V493 Aql & DCEP & 0.4753 & 8.464 & 8.001 & 7.817 & 0.03 & 0.00 & $11.52 \pm 0.07$ & $6344 \pm 447$ & $\mathrm{c}$ & LIII \\
\hline V496 Aql & DCEPS & 0.8330 & 5.564 & 5.136 & 4.966 & 0.05 & 0.11 & $10.68 \pm 0.05$ & $6775 \pm 450$ & b & LII \\
\hline V526 Aql & CEP & 0.6244 & 9.609 & 9.174 & 8.981 & 0.50 & 0.47 & $13.20 \pm 0.07$ & $5481 \pm 386$ & $\mathrm{c}$ & LIII \\
\hline V600 Aql & DCEP & 0.8596 & 6.960 & 6.447 & 6.206 & 0.03 & 0.09 & $11.13 \pm 0.05$ & $6827 \pm 446$ & $\mathrm{a}$ & LII \\
\hline V733 Aql & DCEP & 0.7909 & 8.333 & 7.936 & 7.851 & 0.08 & 0.14 & $12.72 \pm 0.07$ & $6329 \pm 410$ & $\mathrm{c}$ & LII \\
\hline V916 Aql & DCEP & 1.1284 & 7.367 & 6.764 & 6.515 & 0.39 & 0.36 & $12.28 \pm 0.05$ & $6311 \pm 428$ & $\mathrm{a}$ & LIII \\
\hline$\eta$ Aql & DCEP & 0.8559 & 2.388 & 2.062 & 1.945 & 0.08 & 0.14 & $7.07 \pm 0.05$ & $7750 \pm 452$ & b & LII \\
\hline AN Aur & DCEP & 1.0124 & 7.927 & 7.446 & 7.237 & -0.10 & -0.13 & $12.72 \pm 0.05$ & $11355 \pm 458$ & $\mathrm{a}$ & LIII \\
\hline CO Aur & $\mathrm{CEP}(\mathrm{B})$ & 0.2514 & 6.314 & 6.004 & 5.873 & -0.01 & -0.04 & $8.97 \pm 0.13$ & $8557 \pm 453$ & $\mathrm{c}^{*}$ & LIII \\
\hline CY Aur & DCEP & 1.1414 & 8.586 & 7.958 & 7.709 & -0.12 & -0.15 & $13.49 \pm 0.05$ & $12755 \pm 463$ & $\mathrm{a}$ & LIII \\
\hline ER Aur & DCEP & 1.1958 & 9.062 & 8.601 & 8.410 & -0.27 & -0.30 & $14.54 \pm 0.05$ & $15884 \pm 486$ & $\mathrm{a}$ & LIII \\
\hline EW Aur & DCEP & 0.4248 & 11.232 & 10.755 & 10.592 & -0.54 & -0.57 & $14.13 \pm 0.06$ & $14506 \pm 490$ & $\mathrm{c}$ & LIII \\
\hline FF Aur & DCEP & 0.3264 & 11.661 & 11.070 & 10.907 & -0.51 & -0.54 & $14.02 \pm 0.06$ & $14192 \pm 486$ & $\mathrm{c}$ & LIII \\
\hline GT Aur & DCEP & 0.6439 & 10.407 & 10.083 & 9.978 & -0.02 & -0.05 & $14.40 \pm 0.07$ & $15355 \pm 502$ & $\mathrm{c}$ & LIII \\
\hline GV Aur & DCEP & 0.7210 & 9.493 & 9.011 & 8.811 & -0.21 & -0.24 & $13.32 \pm 0.07$ & $12513 \pm 473$ & $\mathrm{c}$ & LIII \\
\hline IN Aur & DCEP & 0.6912 & 10.458 & 9.904 & 9.693 & -0.28 & -0.31 & $14.04 \pm 0.06$ & $14305 \pm 488$ & $\mathrm{c}$ & LIII \\
\hline RT Aur & DCEP & 0.5715 & 4.222 & 3.987 & 3.881 & 0.13 & 0.10 & $8.13 \pm 0.05$ & $8356 \pm 452$ & $\mathrm{a}$ & LIII \\
\hline RX Aur & DCEP & 1.0654 & 5.726 & 5.357 & 5.212 & 0.10 & 0.07 & $10.99 \pm 0.05$ & $9480 \pm 453$ & $\mathrm{a}$ & LIII \\
\hline V335 Aur & DCEP & 0.5331 & 9.863 & 9.398 & 9.195 & -0.30 & -0.33 & $13.08 \pm 0.05$ & $12037 \pm 461$ & $\mathrm{a}$ & LIII \\
\hline V637 Aur & DCEPS & 0.8949 & 8.519 & 8.142 & 7.955 & -0.15 & -0.18 & $13.91 \pm 0.09$ & $13955 \pm 510$ & $\mathrm{c}^{*}$ & LIII \\
\hline AB Cam & DCEP & 0.7625 & 9.418 & 8.936 & 8.746 & -0.08 & -0.11 & $13.40 \pm 0.06$ & $12132 \pm 458$ & $\mathrm{c}$ & LIII \\
\hline AC Cam & DCEP & 0.6188 & 9.325 & 8.752 & 8.499 & -0.13 & -0.16 & $12.56 \pm 0.06$ & $10675 \pm 451$ & c & LIII \\
\hline AD Cam & DCEP & 1.0516 & 8.988 & 8.363 & 8.109 & -0.25 & -0.28 & $13.59 \pm 0.07$ & $12444 \pm 460$ & c & LIII \\
\hline AM Cam & CEP & 0.6018 & 10.179 & 9.517 & 9.313 & -0.13 & -0.16 & $13.28 \pm 0.07$ & $11785 \pm 456$ & $\mathrm{c}$ & LIII \\
\hline CK Cam & DCEP & 0.5172 & 0.000 & 0.000 & 0.000 & 0.07 & 0.04 & $8.93 \pm 0.13$ & $8486 \pm 453$ & $\mathrm{c}$ & LIII \\
\hline LO Cam & DCEP & 1.1017 & 0.000 & 0.000 & 0.000 & -0.05 & -0.08 & $13.03 \pm 0.23$ & $11487 \pm 587$ & $\mathrm{c}$ & LIII \\
\hline MN Cam & DCEP & 0.9138 & 8.219 & 7.609 & 7.348 & 0.01 & -0.02 & $12.37 \pm 0.07$ & $10578 \pm 454$ & $\mathrm{c}$ & LIII \\
\hline MQ Cam & DCEP & 0.8195 & 9.255 & 8.586 & 8.310 & -0.11 & -0.14 & $12.96 \pm 0.06$ & $11447 \pm 457$ & $\mathrm{c}$ & LIII \\
\hline OX Cam & DCEP & 0.7050 & 7.329 & 6.740 & 6.478 & -0.01 & -0.04 & $10.82 \pm 0.06$ & $9152 \pm 451$ & $\mathrm{c}$ & LIII \\
\hline PV Cam & DCEPS: & 0.4900 & 9.749 & 9.341 & 9.170 & -0.17 & -0.20 & $13.71 \pm 0.08$ & $12872 \pm 476$ & $\mathrm{c}^{*}$ & LIII \\
\hline QS Cam & DCEP & 0.7093 & 9.373 & 8.804 & 8.622 & -0.22 & -0.25 & $13.03 \pm 0.07$ & $11530 \pm 458$ & $\mathrm{c}$ & LIII \\
\hline RW Cam & DCEP & 1.2152 & 5.817 & 5.289 & 5.077 & 0.11 & 0.08 & $11.21 \pm 0.05$ & $9414 \pm 451$ & $\mathrm{a}$ & LIII \\
\hline RX Cam & DCEP & 0.8983 & 5.161 & 4.726 & 4.540 & 0.11 & 0.08 & $9.69 \pm 0.05$ & $8668 \pm 451$ & $\mathrm{a}$ & LIII \\
\hline TV Cam & DCEP & 0.7239 & 9.345 & 8.822 & 8.653 & 0.04 & 0.01 & $13.16 \pm 0.07$ & $11682 \pm 457$ & $\mathrm{c}$ & LIII \\
\hline V359 Cam & DCEP & 0.8195 & 9.557 & 9.078 & 8.864 & -0.16 & -0.19 & $13.70 \pm 0.06$ & $13026 \pm 469$ & $\mathrm{c}$ & LIII \\
\hline $\mathrm{CN}$ Car & DCEP & 0.6931 & 8.345 & 7.848 & 7.704 & 0.21 & 0.18 & $12.14 \pm 0.07$ & $7761 \pm 426$ & $\mathrm{c}$ & LIII \\
\hline CY Car & DCEP & 0.6300 & 7.949 & 7.565 & 7.430 & 0.11 & 0.08 & $11.74 \pm 0.07$ & $7496 \pm 434$ & $\mathrm{c}$ & LIII \\
\hline DY Car & DCEP & 0.6697 & 9.160 & 8.729 & 8.563 & 0.07 & 0.04 & $12.96 \pm 0.07$ & $7635 \pm 396$ & $\mathrm{c}$ & LIII \\
\hline ER Car & DCEP & 0.8875 & 5.360 & 5.020 & 4.899 & 0.15 & 0.12 & $10.12 \pm 0.05$ & $7641 \pm 448$ & $\mathrm{~b}^{\dagger}$ & LIII \\
\hline EY Car & DCEP & 0.4588 & 8.352 & 7.953 & 7.808 & -0.17 & -0.18 & $11.53 \pm 0.07$ & $7563 \pm 437$ & $\mathrm{c}$ & SZI \\
\hline FI Car & DCEP & 1.1290 & 8.350 & 7.797 & 7.502 & 0.31 & 0.28 & $13.28 \pm 0.07$ & $7845 \pm 380$ & $\mathrm{c}$ & LIII \\
\hline FR Car & DCEP & 1.0301 & 7.470 & 7.012 & 6.835 & 0.11 & 0.08 & $12.41 \pm 0.05$ & $7410 \pm 417$ & $\mathrm{~b}$ & LIII \\
\hline GH Car & DCEPS & 0.7578 & 7.188 & 6.872 & 6.668 & 0.22 & 0.19 & $12.18 \pm 0.09$ & $7416 \pm 424$ & $\mathrm{c}^{*}$ & LIII \\
\hline GX Car & DCEP & 0.8571 & 7.197 & 6.763 & 6.555 & 0.14 & 0.11 & $11.55 \pm 0.07$ & $7791 \pm 437$ & c & LIII \\
\hline GZ Car & DCEPS(B) & 0.6190 & 8.126 & 7.698 & 7.528 & -0.07 & -0.08 & $11.75 \pm 0.11$ & $7681 \pm 433$ & $\mathrm{c}^{*}$ & SZI \\
\hline HQ Car & DCEP & 1.1483 & 10.791 & 10.348 & 10.185 & -0.41 & -0.10 & $16.18 \pm 0.07$ & $16865 \pm 471$ & $\mathrm{c}$ & YON \\
\hline HW Car & DCEP & 0.9638 & 7.239 & 6.814 & 6.663 & 0.09 & 0.06 & $12.06 \pm 0.07$ & $7567 \pm 427$ & $\mathrm{c}$ & LIII \\
\hline IO Car & CEP & 1.1337 & 8.649 & 8.133 & 7.940 & 0.13 & 0.10 & $13.82 \pm 0.08$ & $8134 \pm 343$ & $\mathrm{c}$ & LIII \\
\hline IT Car & DCEP & 0.8770 & 6.309 & 5.924 & 5.779 & 0.14 & 0.11 & $10.92 \pm 0.05$ & $7517 \pm 444$ & $\mathrm{~b}^{\dagger}$ & LIII \\
\hline SX Car & DCEP & 0.6866 & 7.222 & 6.853 & 6.752 & 0.05 & 0.02 & $11.29 \pm 0.07$ & $7619 \pm 440$ & $\mathrm{c}$ & LIII \\
\hline UW Car & DCEP & 0.7280 & 7.356 & 6.947 & 6.760 & 0.09 & 0.06 & $11.36 \pm 0.07$ & $7652 \pm 439$ & c & LIII \\
\hline UY Car & DCEP & 0.7438 & 7.248 & 6.887 & 6.821 & 0.13 & 0.10 & $11.57 \pm 0.07$ & $7589 \pm 436$ & $\mathrm{c}$ & LIII \\
\hline UZ Car & DCEP & 0.7164 & 7.646 & 7.257 & 7.110 & 0.13 & 0.10 & $11.70 \pm 0.07$ & $7583 \pm 434$ & c & LIII \\
\hline V701 Car & $\mathrm{CEP}(\mathrm{B})$ & 0.6117 & 8.321 & 7.799 & 7.568 & -0.11 & -0.12 & $11.66 \pm 0.16$ & $7769 \pm 435$ & $\mathrm{c}^{*}$ & SZI \\
\hline WW Car & DCEP & 0.6699 & 7.723 & 7.338 & 7.186 & 0.00 & -0.03 & $11.62 \pm 0.07$ & $7551 \pm 435$ & $\mathrm{c}$ & LIII \\
\hline XX Car & DCEP & 1.1963 & 7.176 & 6.745 & 6.566 & 0.20 & 0.17 & $12.72 \pm 0.05$ & $7423 \pm 406$ & $\mathrm{~b}$ & LIII \\
\hline XY Car & DCEP & 1.0946 & 6.915 & 6.419 & 6.227 & 0.07 & 0.04 & $11.99 \pm 0.05$ & $7402 \pm 429$ & $\mathrm{~b}$ & LIII \\
\hline XZ Car & DCEP & 1.2214 & 6.251 & 5.759 & 5.572 & 0.19 & 0.16 & $11.76 \pm 0.05$ & $7463 \pm 433$ & $\mathrm{~b}$ & LIII \\
\hline Y Car & $\operatorname{DCEP}(\mathrm{B})$ & 0.5611 & 6.802 & 6.573 & 6.477 & -0.05 & -0.06 & $10.69 \pm 0.19$ & $7682 \pm 445$ & $c^{*}$ & SZI \\
\hline
\end{tabular}

Notes. From left to right the columns give the same quantities as in Table 3. Column 7 gives the original iron estimate available in the literature, while Col. 8 gives the iron abundance rescaled to current metallicity scale. In Col. 2 the GCVS classification type is listed. We note that V556 Cas and V1397 Cyg are misclassified in the GCVS since they have been identified as classical Cepheids by Wils \& Greaves (2004). Moreover, their nature was confirmed by Luck \& Lambert (2011) on the basis of spectroscopic evidence. The acronym SZI refers to Sziládi et al. (2007) and YON to Yong et al. (2006). 
K. Genovali et al.: The fine structure of the metallicity gradient

Table 4. continued.

\begin{tabular}{|c|c|c|c|c|c|c|c|c|c|c|c|}
\hline Name & Type & $\begin{array}{l}\log (P) \\
\text { [days] }\end{array}$ & $\begin{array}{c}\langle J\rangle \\
\mathrm{mag}\end{array}$ & $\begin{array}{l}\langle H\rangle \\
\text { mag }\end{array}$ & $\begin{array}{l}\langle K\rangle \\
\text { mag }\end{array}$ & {$[\mathrm{Fe} / \mathrm{H}]_{\mathrm{lit}}$} & {$[\mathrm{Fe} / \mathrm{H}]$} & $\begin{array}{c}\mu^{d} \\
\mathrm{mag}\end{array}$ & $\begin{array}{l}R_{\mathrm{G}}^{\mathrm{e}} \\
(\mathrm{pc})\end{array}$ & Notes & $\operatorname{Ref}^{f}$ \\
\hline YZ Car & DCEP & 1.2592 & 6.432 & 5.985 & 5.800 & 0.00 & -0.03 & $12.15 \pm 0.05$ & $7668 \pm 425$ & $\mathrm{~b}$ & LIII \\
\hline AP Cas & DCEP & 0.8355 & 8.788 & 8.189 & 7.982 & 0.05 & 0.02 & $12.79 \pm 0.07$ & $10269 \pm 440$ & c & LIII \\
\hline AS Cas & CEP(B) & 0.4802 & 9.404 & 8.837 & 8.622 & 0.02 & -0.01 & $12.25 \pm 0.15$ & $9662 \pm 458$ & $\mathrm{c}^{*}$ & LIII \\
\hline AW Cas & DCEP & 0.6313 & 8.774 & 8.206 & 7.919 & 0.03 & 0.00 & $12.01 \pm 0.07$ & $9637 \pm 445$ & $\mathrm{c}$ & LIII \\
\hline AY Cas & DCEP & 0.4581 & 9.055 & 8.545 & 8.315 & 0.02 & -0.01 & $11.90 \pm 0.07$ & $9602 \pm 446$ & $\mathrm{c}$ & LIII \\
\hline BF Cas & DCEP & 0.5599 & 9.568 & 9.032 & 8.840 & -0.05 & -0.08 & $12.77 \pm 0.06$ & $10142 \pm 436$ & c & LIII \\
\hline BP Cas & DCEP & 0.7975 & 7.842 & 7.275 & 7.042 & 0.09 & 0.06 & $11.72 \pm 0.07$ & $9392 \pm 446$ & $\mathrm{c}$ & LIII \\
\hline BV Cas & DCEP & 0.7324 & 9.015 & 8.394 & 8.072 & 0.02 & -0.01 & $12.44 \pm 0.07$ & $10113 \pm 445$ & c & LIII \\
\hline BY Cas & DCEPS & 0.5083 & 7.637 & 7.199 & 7.035 & 0.12 & 0.09 & $11.61 \pm 0.12$ & $9420 \pm 453$ & $\mathrm{c}^{*}$ & LIII \\
\hline CD Cas & DCEP & 0.8921 & 7.631 & 7.090 & 6.860 & 0.13 & 0.10 & $11.88 \pm 0.05$ & $9216 \pm 441$ & $\mathrm{a}$ & LIII \\
\hline CF Cas & DCEP & 0.6880 & 8.590 & 8.126 & 7.900 & 0.02 & -0.01 & $12.29 \pm 0.05$ & $9579 \pm 437$ & $\mathrm{a}$ & LIII \\
\hline CG Cas & DCEP & 0.6400 & 8.903 & 8.299 & 8.109 & 0.09 & 0.06 & $12.26 \pm 0.07$ & $9557 \pm 440$ & $\mathrm{c}$ & LIII \\
\hline $\mathrm{CH}$ Cas & DCEP & 1.1786 & 7.331 & 6.686 & 6.385 & 0.17 & 0.23 & $12.25 \pm 0.05$ & $9405 \pm 436$ & a & LII \\
\hline CT Cas & DCEP & 0.5810 & 9.455 & 8.810 & 8.600 & -0.05 & -0.08 & $12.51 \pm 0.07$ & $9892 \pm 439$ & $\mathrm{c}$ & LIII \\
\hline CY Cas & DCEP & 1.1577 & 7.861 & 7.174 & 6.903 & 0.06 & 0.12 & $12.69 \pm 0.05$ & $9849 \pm 432$ & $\mathrm{a}$ & LII \\
\hline CZ Cas & DCEP & 0.7532 & 8.622 & 8.124 & 7.855 & 0.07 & 0.04 & $12.42 \pm 0.07$ & $9621 \pm 437$ & $\mathrm{c}$ & LIII \\
\hline DD Cas & DCEP & 0.9918 & 7.526 & 7.067 & 6.887 & 0.10 & 0.16 & $12.33 \pm 0.05$ & $9623 \pm 437$ & a & LII \\
\hline DF Cas & DCEP & 0.5835 & 8.488 & 8.036 & 7.879 & 0.13 & 0.19 & $11.97 \pm 0.07$ & $9873 \pm 449$ & c & LII \\
\hline DL Cas & DCEP & 0.9031 & 6.550 & 6.101 & 5.892 & -0.01 & 0.05 & $11.03 \pm 0.05$ & $8859 \pm 447$ & $\mathrm{a}$ & LII \\
\hline DW Cas & DCEP & 0.6988 & 8.195 & 7.671 & 7.420 & 0.06 & 0.03 & $11.79 \pm 0.07$ & $9103 \pm 442$ & $\mathrm{c}$ & LIII \\
\hline EX Cas & DCEP & 0.6340 & 9.556 & 8.944 & 8.742 & -0.07 & -0.10 & $12.86 \pm 0.07$ & $10202 \pm 435$ & $\mathrm{c}$ & LIII \\
\hline FM Cas & DCEP & 0.7641 & 7.128 & 6.747 & 6.581 & -0.09 & -0.03 & $11.33 \pm 0.05$ & $8942 \pm 445$ & a & LII \\
\hline FO Cas & DCEP & 0.8324 & 11.136 & 10.529 & 10.267 & -0.56 & -0.59 & $15.02 \pm 0.06$ & $15552 \pm 459$ & $\mathrm{c}$ & LIII \\
\hline FW Cas & DCEP & 0.7950 & 9.408 & 8.716 & 8.393 & -0.09 & -0.12 & $12.92 \pm 0.07$ & $10460 \pm 440$ & $\mathrm{c}$ & LIII \\
\hline GL Cas & DCEP & 0.6029 & 9.778 & 9.432 & 9.216 & 0.03 & 0.00 & $13.42 \pm 0.07$ & $11773 \pm 449$ & $\mathrm{c}$ & LIII \\
\hline GM Cas & DCEP & 0.8733 & 7.823 & 7.135 & 6.870 & -0.10 & -0.13 & $11.70 \pm 0.06$ & $9596 \pm 449$ & $\mathrm{c}$ & LIII \\
\hline GO Cas & DCEP & 0.5104 & 9.718 & 9.160 & 8.898 & 0.12 & 0.09 & $12.61 \pm 0.06$ & $10575 \pm 449$ & $\mathrm{c}$ & LIII \\
\hline HK Cas & DCEP & 0.3979 & 12.016 & 11.831 & 11.668 & 0.45 & 0.42 & $15.34 \pm 0.07$ & $17296 \pm 498$ & $\mathrm{c}$ & LIII \\
\hline IO Cas & DCEP & 0.7485 & 11.082 & 10.612 & 10.503 & -0.49 & -0.52 & $15.16 \pm 0.07$ & $16998 \pm 499$ & $\mathrm{c}$ & LIII \\
\hline KK Cas & DCEP & 0.9134 & 8.632 & 7.998 & 7.774 & 0.13 & 0.10 & $12.80 \pm 0.07$ & $10043 \pm 434$ & $\mathrm{c}$ & LIII \\
\hline LT Cas & DCEP & 0.7712 & 9.733 & 9.264 & 9.074 & -0.36 & -0.39 & $13.77 \pm 0.06$ & $12609 \pm 454$ & $\mathrm{c}$ & LIII \\
\hline NP Cas & DCEP & 0.7903 & 9.584 & 8.876 & 8.550 & 0.01 & -0.02 & $13.05 \pm 0.07$ & $10572 \pm 436$ & $\mathrm{c}$ & LIII \\
\hline NY Cas & DCEPS & 0.4507 & 11.398 & 10.985 & 10.850 & -0.46 & -0.49 & $15.27 \pm 0.12$ & $16839 \pm 693$ & $\mathrm{c}^{*}$ & LIII \\
\hline OP Cas & DCEP & 0.7414 & 8.778 & 8.207 & 7.966 & 0.14 & 0.11 & $12.45 \pm 0.07$ & $9945 \pm 441$ & $\mathrm{c}$ & LIII \\
\hline OZ Cas & DCEP & 0.7059 & 8.491 & 7.482 & 7.074 & 0.06 & 0.03 & $11.01 \pm 0.06$ & $8962 \pm 448$ & $\mathrm{c}$ & LIII \\
\hline PW Cas & DCEP & 0.6021 & 10.246 & 9.679 & 9.483 & -0.06 & -0.09 & $13.53 \pm 0.07$ & $10959 \pm 425$ & $\mathrm{c}$ & LIII \\
\hline RS Cas & DCEP & 0.7991 & 6.769 & 6.225 & 5.968 & 0.18 & 0.15 & $10.66 \pm 0.05$ & $8589 \pm 447$ & a & LIII \\
\hline RW Cas & DCEP & 1.1701 & 6.829 & 6.366 & 6.175 & 0.22 & 0.28 & $12.21 \pm 0.05$ & $9914 \pm 444$ & $\mathrm{a}$ & LII \\
\hline RY Cas & DCEP & 1.0841 & 7.062 & 6.549 & 6.311 & 0.26 & 0.32 & $11.99 \pm 0.05$ & $9288 \pm 440$ & $\mathrm{a}$ & LII \\
\hline SU Cas & DCEPS & 0.2899 & 4.483 & 4.210 & 4.114 & 0.06 & 0.12 & $8.10 \pm 0.05$ & $8229 \pm 451$ & $\mathrm{~b}$ & LII \\
\hline SW Cas & DCEP & 0.7357 & 7.400 & 6.983 & 6.801 & -0.03 & 0.03 & $11.42 \pm 0.05$ & $8774 \pm 443$ & a & LII \\
\hline SY Cas & DCEP & 0.6097 & 7.816 & 7.449 & 7.287 & 0.04 & 0.10 & $11.53 \pm 0.05$ & $9068 \pm 444$ & a & LII \\
\hline SZ Cas & DCEPS & 1.1346 & 6.814 & 6.217 & 6.007 & 0.07 & 0.04 & $12.61 \pm 0.10$ & $10557 \pm 457$ & $\mathrm{c}^{*}$ & LIII \\
\hline TU Cas & CEP(B) & 0.3303 & 6.681 & 6.441 & 6.331 & 0.03 & 0.09 & $9.76 \pm 0.25$ & $8398 \pm 453$ & $\mathrm{c}^{*}$ & LII \\
\hline UZ Cas & DCEP & 0.6294 & 9.155 & 8.679 & 8.522 & -0.05 & -0.08 & $12.75 \pm 0.06$ & $10408 \pm 442$ & $\mathrm{c}$ & LIII \\
\hline V1017 Cas & DCEP & 0.6665 & 9.766 & 9.289 & 9.100 & -0.18 & -0.21 & $13.43 \pm 0.06$ & $11725 \pm 447$ & c & LIII \\
\hline V1019 Cas & DCEPS & 0.5587 & 8.390 & 7.813 & 7.566 & 0.07 & 0.04 & $12.17 \pm 0.07$ & $9312 \pm 438$ & $\mathrm{c}^{*}$ & LIII \\
\hline V1020 Cas & DCEP & 0.6758 & 9.286 & 8.569 & 8.276 & 0.15 & 0.12 & $12.40 \pm 0.07$ & $9580 \pm 437$ & $\mathrm{c}$ & LIII \\
\hline V1100 Cas & DCEP & 0.6730 & 8.991 & 8.351 & 8.088 & 0.02 & -0.01 & $12.28 \pm 0.07$ & $9899 \pm 444$ & c & LIII \\
\hline V1154 Cas & DCEP & 0.3249 & 8.933 & 8.554 & 8.453 & -0.06 & -0.09 & $11.76 \pm 0.07$ & $9508 \pm 447$ & $\mathrm{c}$ & LIII \\
\hline V1206 Cas & DCEP & 0.6758 & 9.419 & 8.871 & 8.655 & -0.16 & -0.19 & $12.95 \pm 0.06$ & $10239 \pm 432$ & $\mathrm{c}$ & LIII \\
\hline V342 Cas & DCEP & 0.5933 & 9.010 & 8.510 & 8.327 & 0.03 & 0.00 & $12.40 \pm 0.06$ & $9355 \pm 433$ & c & LIII \\
\hline V379 Cas & DCEPS & 0.6340 & 6.816 & 6.362 & 6.217 & 0.06 & 0.12 & $11.23 \pm 0.09$ & $8950 \pm 447$ & $\mathrm{c}^{*}$ & LII \\
\hline V395 Cas & DCEP & 0.6061 & 8.446 & 8.001 & 7.874 & 0.02 & -0.01 & $12.06 \pm 0.07$ & $9849 \pm 447$ & $\mathrm{c}$ & LIII \\
\hline V407 Cas & DCEP & 0.6613 & 9.404 & 8.890 & 8.673 & 0.11 & 0.08 & $12.95 \pm 0.07$ & $10270 \pm 433$ & c & LIII \\
\hline V556 Cas & RR: & 0.7803 & 8.883 & 8.292 & 7.984 & -0.01 & -0.04 & $12.54 \pm 0.29$ & $10201 \pm 549$ & $c^{*}$ & LIII \\
\hline V636 Cas & DCEPS & 0.9231 & 4.899 & 4.158 & 4.059 & 0.07 & 0.13 & $9.83 \pm 0.29$ & $8533 \pm 458$ & $\mathrm{c}^{*}$ & LII \\
\hline VV Cas & DCEP & 0.7929 & 8.316 & 7.880 & 7.697 & -0.04 & -0.07 & $12.49 \pm 0.05$ & $10260 \pm 443$ & $\mathrm{a}$ & LIII \\
\hline VW Cas & DCEP & 0.7777 & 8.343 & 7.844 & 7.675 & 0.19 & 0.16 & $12.38 \pm 0.07$ & $9947 \pm 443$ & $\mathrm{c}$ & LIII \\
\hline XY Cas & DCEP & 0.6534 & 7.735 & 7.336 & 7.156 & 0.10 & 0.07 & $11.51 \pm 0.05$ & $9178 \pm 445$ & $\mathrm{a}$ & LIII \\
\hline AY Cen & DCEP & 0.7251 & 6.808 & 6.431 & 6.249 & 0.08 & 0.05 & $10.86 \pm 0.07$ & $7496 \pm 444$ & c & LIII \\
\hline AZ Cen & DCEPS & 0.5066 & 7.222 & 6.944 & 6.831 & 0.09 & 0.06 & $11.55 \pm 0.05$ & $7392 \pm 437$ & $\mathrm{~b}$ & LIII \\
\hline BB Cen & DCEPS & 0.6018 & 7.922 & 7.539 & 7.369 & 0.22 & 0.19 & $12.31 \pm 0.13$ & $7140 \pm 421$ & $\mathrm{c}^{*}$ & LIII \\
\hline BK Cen & CEP(B) & 0.5016 & 8.339 & 7.947 & 7.772 & 0.07 & 0.06 & $11.62 \pm 0.16$ & $7268 \pm 437$ & $\mathrm{c}^{*}$ & SZI \\
\hline KK Cen & DCEP & 1.0857 & 8.713 & 8.117 & 7.955 & 0.24 & 0.21 & $13.62 \pm 0.07$ & $7526 \pm 349$ & c & LIII \\
\hline QY Cen & DCEP & 1.2493 & 7.374 & 6.596 & 6.242 & 0.24 & 0.21 & $12.22 \pm 0.07$ & $6427 \pm 429$ & c & LIII \\
\hline UZ Cen & CEP(B) & 0.5230 & 7.335 & 6.957 & 6.767 & -0.19 & -0.20 & $10.69 \pm 0.16$ & $7465 \pm 446$ & $\mathrm{c}^{*}$ & SZI \\
\hline V1048 Cen & CEP(B) & -0.0351 & 9.113 & 8.799 & 8.719 & -0.18 & -0.19 & $10.88 \pm 0.08$ & $7493 \pm 444$ & $\mathrm{c}^{*}$ & SZI \\
\hline V1210 Cen & CEP(B) & 0.6352 & 7.603 & 7.173 & 6.968 & -0.02 & -0.03 & $11.23 \pm 0.06$ & $6775 \pm 445$ & $\mathrm{c}^{*}$ & SZI \\
\hline V378 Cen & DCEPS & 0.8102 & 6.328 & 5.909 & 5.743 & 0.08 & 0.05 & $11.38 \pm 0.05$ & $6993 \pm 441$ & $\mathrm{~b}^{\dagger}$ & LIII \\
\hline V381 Cen & CEP & 0.7058 & 6.143 & 5.822 & 5.703 & 0.02 & -0.01 & $10.33 \pm 0.05$ & $7235 \pm 449$ & $\mathrm{~b}^{\dagger}$ & LIII \\
\hline v419 Cen & DCEPS & 0.7409 & 6.706 & 6.461 & 6.273 & 0.14 & 0.11 & $11.79 \pm 0.09$ & $7392 \pm 433$ & $\mathrm{c}^{*}$ & LIII \\
\hline V496 Cen & DCEP & 0.6458 & 7.424 & 6.977 & 6.784 & 0.09 & 0.06 & $11.07 \pm 0.07$ & $7145 \pm 444$ & $\mathrm{c}$ & LIII \\
\hline V659 Cen & DCEP & 0.7499 & 5.177 & 4.907 & 4.651 & 0.09 & 0.06 & $9.38 \pm 0.07$ & $7504 \pm 451$ & c & LIII \\
\hline V737 Cen & DCEP & 0.8492 & 4.951 & 4.573 & 4.432 & 0.14 & 0.11 & $9.48 \pm 0.05$ & $7403 \pm 451$ & $\mathrm{~b}^{\dagger}$ & LIII \\
\hline AK Cep & DCEP & 0.8593 & 8.400 & 7.896 & 7.703 & 0.05 & 0.02 & $12.66 \pm 0.05$ & $9420 \pm 426$ & $\mathrm{a}$ & LIII \\
\hline CN Cep & DCEP & 0.9778 & 8.329 & 7.641 & 7.333 & 0.06 & 0.03 & $12.49 \pm 0.05$ & $9649 \pm 434$ & $\mathrm{a}$ & LIII \\
\hline CP Cep & DCEP & 1.2519 & 7.335 & 6.724 & 6.476 & -0.01 & 0.05 & $12.64 \pm 0.05$ & $9175 \pm 423$ & $\mathrm{a}$ & LII \\
\hline CR Cep & DCEP & 0.7947 & 6.641 & 6.097 & 5.873 & -0.06 & -0.00 & $10.57 \pm 0.05$ & $8424 \pm 447$ & a & LII \\
\hline DR Cep & DCEP & 1.2803 & 9.471 & 8.851 & 8.695 & -0.14 & -0.17 & $15.00 \pm 0.07$ & $13289 \pm 389$ & $\mathrm{c}$ & LIII \\
\hline
\end{tabular}


Table 4. continued.

\begin{tabular}{|c|c|c|c|c|c|c|c|c|c|c|c|}
\hline Name & Type & $\begin{array}{l}\log (P) \\
\text { [days] }\end{array}$ & $\begin{array}{c}\langle J\rangle \\
\mathrm{mag}\end{array}$ & $\begin{array}{l}\langle H\rangle \\
\mathrm{mag}\end{array}$ & $\begin{array}{l}\langle K\rangle \\
\mathrm{mag}\end{array}$ & {$[\mathrm{Fe} / \mathrm{H}]_{\text {lit }}$} & {$[\mathrm{Fe} / \mathrm{H}]$} & $\begin{array}{c}\mu^{d} \\
\mathrm{mag}\end{array}$ & $\begin{array}{l}R_{\mathrm{G}}^{\mathrm{e}} \\
(\mathrm{pc})\end{array}$ & Notes & $\operatorname{Ref}^{f}$ \\
\hline IR Cep & DCEP & 0.3251 & 5.907 & 5.593 & 5.454 & 0.05 & 0.11 & $9.58 \pm 0.09$ & $8169 \pm 450$ & $\mathrm{c}$ & LII \\
\hline IY Cep & CEP & 0.7526 & 9.871 & 9.123 & 8.843 & 0.11 & 0.08 & $13.21 \pm 0.06$ & $9777 \pm 412$ & c & LIII \\
\hline MU Cep & CEP & 0.5761 & 9.370 & 8.783 & 8.632 & 0.18 & 0.15 & $12.60 \pm 0.07$ & $9321 \pm 428$ & c & LIII \\
\hline V901 Cep & DCEP & 0.9542 & 8.022 & 7.351 & 7.073 & 0.04 & 0.01 & $12.18 \pm 0.07$ & $9138 \pm 436$ & $\mathrm{c}$ & LIII \\
\hline V911 Cep & DCEP & 0.6312 & 8.854 & 8.220 & 7.977 & -0.02 & -0.05 & $12.04 \pm 0.07$ & $9076 \pm 437$ & $\mathrm{c}$ & LIII \\
\hline$\delta$ Cep & DCEP & 0.7297 & 2.700 & 2.397 & 2.306 & 0.12 & 0.09 & $7.04 \pm 0.05$ & $8010 \pm 452$ & b & LIII \\
\hline AV Cir & DCEP & 0.4864 & 5.582 & 5.236 & 5.093 & 0.17 & 0.14 & $9.68 \pm 0.05$ & $7371 \pm 450$ & $\mathrm{~b}^{\dagger}$ & LIII \\
\hline $\mathrm{AX} \mathrm{Cir}$ & DCEP & 0.7221 & 4.299 & 3.879 & 3.780 & -0.01 & -0.04 & $8.41 \pm 0.17$ & $7603 \pm 452$ & $\mathrm{c}$ & LIII \\
\hline BP Cir & CEP & 0.3799 & 5.918 & 5.612 & 5.483 & 0.02 & -0.01 & $9.74 \pm 0.05$ & $7326 \pm 450$ & $\mathrm{~b}^{\dagger}$ & LIII \\
\hline VZ Cma & DCEPS & 0.4950 & 7.221 & 6.806 & 6.605 & -0.06 & -0.00 & $11.13 \pm 0.10$ & $8903 \pm 449$ & $\mathrm{c}^{*}$ & LII \\
\hline XZ Cma & CEP & 0.4082 & 10.970 & 10.558 & 10.390 & -0.57 & -0.26 & $13.92 \pm 0.07$ & $12567 \pm 447$ & $\mathrm{c}$ & YON \\
\hline AD Cru & DCEP & 0.8060 & 8.198 & 7.661 & 7.387 & 0.11 & 0.08 & $12.10 \pm 0.07$ & $7075 \pm 427$ & $\mathrm{c}$ & LIII \\
\hline AG Cru & DCEP & 0.5840 & 6.674 & 6.353 & 6.230 & 0.08 & 0.05 & $10.44 \pm 0.05$ & $7371 \pm 447$ & b & LIII \\
\hline BG Cru & DCEPS & 0.5241 & 4.237 & 3.975 & 3.878 & -0.08 & -0.11 & $8.68 \pm 0.05$ & $7678 \pm 451$ & b & LIII \\
\hline R Cru & DCEP & 0.7654 & 5.260 & 4.931 & 4.810 & 0.13 & 0.10 & $9.63 \pm 0.05$ & $7559 \pm 450$ & $\mathrm{~b}^{\dagger}$ & LIII \\
\hline S Cru & DCEP & 0.6712 & 5.086 & 4.755 & 4.632 & 0.11 & 0.08 & $9.13 \pm 0.05$ & $7593 \pm 451$ & $\mathrm{~b}$ & LIII \\
\hline $\mathrm{T}$ Cru & DCEP & 0.8282 & 4.941 & 4.541 & 4.407 & 0.14 & 0.11 & $9.37 \pm 0.05$ & $7599 \pm 450$ & $\mathrm{~b}^{\dagger}$ & LIII \\
\hline VW Cru & DCEP & 0.7214 & 6.805 & 6.261 & 6.051 & 0.19 & 0.16 & $10.51 \pm 0.07$ & $7370 \pm 447$ & $\mathrm{c}$ & LIII \\
\hline X Cru & DCEP & 0.7938 & 6.521 & 6.125 & 5.935 & 0.15 & 0.12 & $10.76 \pm 0.07$ & $7283 \pm 446$ & c & LIII \\
\hline BZ Cyg & DCEP & 1.0061 & 6.758 & 6.147 & 5.864 & 0.19 & 0.25 & $11.19 \pm 0.05$ & $7971 \pm 441$ & a & LII \\
\hline CD Cyg & DCEP & 1.2323 & 6.347 & 5.842 & 5.647 & 0.15 & 0.12 & $11.86 \pm 0.05$ & $7513 \pm 431$ & $\mathrm{a}$ & LIII \\
\hline DT Cyg & DCEPS & 0.3978 & 4.638 & 4.390 & 4.374 & 0.10 & 0.16 & $8.80 \pm 0.18$ & $7827 \pm 451$ & $\mathrm{c}^{*}$ & LII \\
\hline EP Cyg & DCEP & 0.6323 & 10.112 & 9.687 & 9.483 & -0.06 & -0.09 & $13.73 \pm 0.05$ & $7674 \pm 339$ & $\mathrm{a}$ & LIII \\
\hline EU Cyg & CEP & 1.1758 & 10.177 & 9.679 & 9.456 & -0.20 & -0.23 & $15.47 \pm 0.06$ & $11847 \pm 314$ & $\mathrm{c}$ & LIII \\
\hline EX Cyg & DCEP & 0.6858 & 9.665 & 9.023 & 8.752 & 0.25 & 0.22 & $12.98 \pm 0.06$ & $7391 \pm 393$ & c & LIII \\
\hline EZ Cyg & DCEP & 1.0667 & 8.190 & 7.667 & 7.460 & 0.28 & 0.25 & $13.10 \pm 0.05$ & $7389 \pm 386$ & $\mathrm{a}$ & LIII \\
\hline GH Cyg & DCEP & 0.8931 & 7.251 & 6.799 & 6.577 & 0.21 & 0.18 & $11.67 \pm 0.05$ & $7351 \pm 435$ & $\mathrm{a}$ & LIII \\
\hline GI Cyg & DCEP & 0.7621 & 8.756 & 8.225 & 7.985 & 0.27 & 0.24 & $12.57 \pm 0.07$ & $7494 \pm 412$ & $\mathrm{c}$ & LIII \\
\hline GL Cyg & CEP & 0.5277 & 11.173 & 10.633 & 10.481 & 0.05 & 0.02 & $14.32 \pm 0.07$ & $9314 \pm 320$ & c & LIII \\
\hline IY Cyg & CEP & 1.3375 & 9.523 & 8.637 & 8.328 & -0.09 & -0.12 & $14.54 \pm 0.06$ & $8976 \pm 283$ & $\mathrm{c}$ & LIII \\
\hline KX Cyg & DCEP & 1.3020 & 6.867 & 5.995 & 5.589 & 0.21 & 0.18 & $11.64 \pm 0.05$ & $7804 \pm 435$ & $\mathrm{a}$ & LIII \\
\hline MW Cyg & DCEP & 0.7749 & 6.688 & 6.206 & 5.984 & 0.09 & 0.15 & $10.66 \pm 0.05$ & $7605 \pm 445$ & $\mathrm{a}$ & LII \\
\hline SU Cyg & DCEP & 0.5850 & 5.629 & 5.390 & 5.268 & -0.03 & 0.03 & $9.55 \pm 0.05$ & $7629 \pm 450$ & $\mathrm{a}$ & LII \\
\hline SZ Cyg & DCEP & 1.1793 & 6.517 & 5.957 & 5.709 & 0.09 & 0.15 & $11.67 \pm 0.05$ & $8023 \pm 435$ & $\mathrm{a}$ & LII \\
\hline TX Cyg & DCEP & 1.1676 & 5.326 & 4.630 & 4.307 & 0.20 & 0.26 & $10.08 \pm 0.05$ & $7905 \pm 448$ & $\mathrm{a}$ & LII \\
\hline V1020 Cyg & DCEP & 0.6920 & 9.697 & 8.958 & 8.655 & 0.29 & 0.26 & $12.81 \pm 0.07$ & $7473 \pm 402$ & c & LIII \\
\hline V1025 Cyg & DCEP & 0.8427 & 9.534 & 8.849 & 8.562 & 0.23 & 0.20 & $13.28 \pm 0.06$ & $7518 \pm 376$ & $\mathrm{c}$ & LIII \\
\hline V1033 Cyg & CEP & 0.6935 & 9.503 & 8.918 & 8.673 & 0.12 & 0.09 & $12.99 \pm 0.07$ & $7559 \pm 394$ & c & LIII \\
\hline V1046 Cyg & CEP & 0.6941 & 8.978 & 8.257 & 8.012 & 0.23 & 0.20 & $12.22 \pm 0.06$ & $7679 \pm 423$ & c & LIII \\
\hline V1154 Cyg & CEP & 0.6925 & 7.457 & 7.094 & 6.964 & -0.10 & -0.04 & $11.51 \pm 0.07$ & $7746 \pm 437$ & c & LII \\
\hline V1334 Cyg & DCEPS & 0.5228 & 5.170 & 4.664 & 4.460 & 0.03 & 0.09 & $9.00 \pm 0.13$ & $7895 \pm 450$ & $\mathrm{c}^{*}$ & LII \\
\hline V1364 Cyg & DCEP & 1.1132 & 9.104 & 8.351 & 8.055 & 0.29 & 0.26 & $13.62 \pm 0.05$ & $8109 \pm 356$ & $\mathrm{a}$ & LIII \\
\hline V1397 Cyg & IS: & 0.6665 & 9.127 & 8.507 & 8.262 & 0.01 & -0.02 & $12.46 \pm 0.27$ & $8874 \pm 459$ & $\mathrm{c}^{*}$ & LIII \\
\hline V1726 Cyg & DCEPS & 0.6269 & 7.181 & 6.836 & 6.699 & -0.02 & 0.04 & $11.78 \pm 0.07$ & $8352 \pm 435$ & $\mathrm{c}^{*}$ & LII \\
\hline V347 Cyg & DCEP & 0.9413 & 8.874 & 8.237 & 7.969 & 0.25 & 0.22 & $13.06 \pm 0.07$ & $8679 \pm 402$ & $\mathrm{c}$ & LIII \\
\hline V356 Cyg & DCEP & 0.7039 & 9.802 & 9.271 & 9.046 & 0.17 & 0.14 & $13.45 \pm 0.07$ & $9203 \pm 390$ & $\mathrm{c}$ & LIII \\
\hline V386 Cyg & DCEP & 0.7208 & 6.363 & 5.806 & 5.524 & 0.11 & 0.17 & $9.93 \pm 0.05$ & $7923 \pm 448$ & $\mathrm{a}$ & LII \\
\hline V396 Cyg & DCEP & 1.5218 & 6.072 & 5.053 & 4.588 & 0.11 & 0.08 & $11.23 \pm 0.05$ & $7799 \pm 440$ & $\mathrm{a}$ & LIII \\
\hline V402 Cyg & DCEP & 0.6400 & 7.799 & 7.411 & 7.247 & 0.02 & 0.08 & $11.57 \pm 0.05$ & $7638 \pm 436$ & $\mathrm{a}$ & LII \\
\hline V438 Cyg & DCEP & 1.0496 & 6.780 & 6.074 & 5.723 & 0.33 & 0.30 & $11.08 \pm 0.05$ & $7755 \pm 442$ & a & LIII \\
\hline V459 Cyg & DCEP & 0.8604 & 7.600 & 7.071 & 6.847 & 0.09 & 0.06 & $11.77 \pm 0.05$ & $8273 \pm 435$ & $\mathrm{a}$ & LIII \\
\hline V492 Cyg & DCEP & 0.8797 & 8.750 & 8.145 & 7.859 & 0.24 & 0.21 & $12.76 \pm 0.07$ & $7688 \pm 405$ & c & LIII \\
\hline V495 Cyg & DCEP & 0.8276 & 7.110 & 6.539 & 6.252 & 0.24 & 0.21 & $11.00 \pm 0.05$ & $7632 \pm 443$ & $\mathrm{a}$ & LIII \\
\hline V514 Cyg & DCEP & 0.7075 & 7.647 & 7.011 & 6.702 & 0.18 & 0.15 & $10.99 \pm 0.05$ & $7957 \pm 443$ & $\mathrm{a}$ & LIII \\
\hline V520 Cyg & DCEP & 0.6074 & 8.229 & 7.755 & 7.590 & 0.08 & 0.05 & $11.74 \pm 0.07$ & $8153 \pm 435$ & c & LIII \\
\hline V532 Cyg & DCEPS & 0.5164 & 6.863 & 6.393 & 6.250 & -0.04 & 0.02 & $10.85 \pm 0.10$ & $8049 \pm 444$ & $\mathrm{c}^{*}$ & LII \\
\hline V538 Cyg & DCEP & 0.7867 & 7.791 & 7.306 & 7.099 & 0.05 & 0.02 & $11.82 \pm 0.05$ & $8473 \pm 435$ & $\mathrm{a}$ & LIII \\
\hline V547 Cyg & DCEP & 0.7942 & 10.065 & 9.530 & 9.316 & 0.15 & 0.12 & $14.02 \pm 0.06$ & $8468 \pm 328$ & c & LIII \\
\hline V609 Cyg & DCEP & 1.4924 & 6.815 & 6.122 & 5.783 & 0.22 & 0.19 & $12.65 \pm 0.05$ & $8949 \pm 420$ & $\mathrm{a}$ & LIII \\
\hline V621 Cyg & DCEP & 0.7682 & 9.367 & 8.735 & 8.540 & 0.12 & 0.09 & $13.10 \pm 0.07$ & $9156 \pm 407$ & $\mathrm{c}$ & LIII \\
\hline V924 Cyg & DCEPS & 0.7460 & 8.963 & 8.654 & 8.511 & -0.09 & -0.03 & $14.03 \pm 0.08$ & $7987 \pm 321$ & $\mathrm{c}^{*}$ & LII \\
\hline VX Cyg & DCEP & 1.3039 & 6.694 & 6.078 & 5.840 & 0.09 & 0.15 & $12.19 \pm 0.05$ & $8037 \pm 426$ & $\mathrm{a}$ & LII \\
\hline VY Cyg & DCEP & 0.8953 & 6.999 & 6.548 & 6.337 & 0.00 & 0.06 & $11.45 \pm 0.05$ & $7937 \pm 438$ & $\mathrm{a}$ & LII \\
\hline VZ Cyg & DCEP & 0.6870 & 7.186 & 6.852 & 6.699 & 0.05 & 0.11 & $11.23 \pm 0.05$ & $8174 \pm 441$ & $\mathrm{a}$ & LII \\
\hline X Cyg & DCEP & 1.2145 & 4.417 & 3.969 & 3.815 & 0.10 & 0.16 & $10.04 \pm 0.05$ & $7772 \pm 448$ & $\mathrm{~b}$ & LII \\
\hline EK Del & CEP & 0.3111 & 11.292 & 10.953 & 10.887 & -1.54 & -1.57 & $14.20 \pm 0.07$ & $6867 \pm 292$ & $\mathrm{c}$ & LIII \\
\hline W Gem & DCEP & 0.8984 & 5.117 & 4.768 & 4.633 & 0.02 & -0.01 & $9.87 \pm 0.05$ & $8843 \pm 452$ & $\mathrm{a}$ & LIII \\
\hline BB Her & DCEP & 0.8755 & 8.076 & 7.675 & 7.538 & 0.26 & 0.23 & $12.66 \pm 0.06$ & $5950 \pm 418$ & c & LIII \\
\hline BG Lac & DCEP & 0.7269 & 7.009 & 6.649 & 6.473 & 0.07 & 0.04 & $11.11 \pm 0.05$ & $8191 \pm 443$ & a & LIII \\
\hline DF Lac & DCEP & 0.6512 & 9.429 & 9.054 & 8.848 & 0.04 & 0.01 & $13.20 \pm 0.07$ & $9834 \pm 415$ & $\mathrm{c}$ & LIII \\
\hline FQ Lac & CEP: & 1.0518 & 11.234 & 10.278 & 9.630 & -0.20 & -0.23 & $14.64 \pm 0.07$ & $13114 \pm 413$ & $\mathrm{c}$ & LIII \\
\hline RR Lac & DCEP & 0.8073 & 6.967 & 6.620 & 6.468 & 0.04 & 0.01 & $11.40 \pm 0.05$ & $8647 \pm 442$ & a & LIII \\
\hline V Lac & DCEP & 0.6975 & 7.034 & 6.667 & 6.531 & 0.06 & 0.03 & $11.08 \pm 0.05$ & $8552 \pm 444$ & $\mathrm{a}$ & LIII \\
\hline V411 Lac & DCEPS & 0.4639 & 6.309 & 6.033 & 5.929 & 0.02 & -0.01 & $10.51 \pm 0.07$ & $8279 \pm 447$ & $\mathrm{c}^{*}$ & LIII \\
\hline X Lac & DCEPS & 0.7360 & 6.492 & 6.139 & 5.969 & 0.08 & 0.05 & $11.40 \pm 0.05$ & $8676 \pm 442$ & $\mathrm{a}$ & LIII \\
\hline Y Lac & DCEP & 0.6359 & 7.612 & 7.310 & 7.180 & 0.03 & 0.00 & $11.58 \pm 0.05$ & $8500 \pm 439$ & $\mathrm{a}$ & LIII \\
\hline Z Lac & DCEP & 1.0369 & 6.210 & 5.804 & 5.630 & 0.10 & 0.07 & $11.27 \pm 0.05$ & $8603 \pm 443$ & $\mathrm{a}$ & LIII \\
\hline V473 Lyr & DCEPS: & 0.1734 & 5.470 & 4.700 & 4.549 & -0.06 & -0.09 & $7.86 \pm 0.18$ & $7764 \pm 452$ & $\mathrm{c}^{*}$ & LIII \\
\hline AA Mon & DCEP & 0.5953 & 9.729 & 9.199 & 8.953 & -0.09 & -0.12 & $12.97 \pm 0.05$ & $11330 \pm 450$ & $\mathrm{a}^{\dagger}$ & LIII \\
\hline AC Mon & DCEP & 0.9039 & 7.577 & 7.066 & 6.849 & -0.03 & -0.06 & $11.94 \pm 0.05$ & $9895 \pm 448$ & $\mathrm{a}$ & LIII \\
\hline
\end{tabular}


Table 4. continued.

\begin{tabular}{|c|c|c|c|c|c|c|c|c|c|c|c|}
\hline Name & Type & $\begin{array}{l}\log (P) \\
\text { [days] }\end{array}$ & $\begin{array}{c}\langle J\rangle \\
\text { mag } \\
\end{array}$ & $\begin{array}{c}\langle H\rangle \\
\mathrm{mag} \\
\end{array}$ & $\begin{array}{c}\langle K\rangle \\
\text { mag } \\
\end{array}$ & {$[\mathrm{Fe} / \mathrm{H}]_{\text {lit }}$} & {$[\mathrm{Fe} / \mathrm{H}]$} & $\begin{array}{c}\mu^{d} \\
\text { mag }\end{array}$ & $\begin{array}{l}R_{\mathrm{G}}^{\mathrm{e}} \\
(\mathrm{pc}) \\
\end{array}$ & Notes & Ref. $^{f}$ \\
\hline CS Mon & CEP & 0.8281 & 8.634 & 8.160 & 7.949 & -0.08 & -0.11 & $12.82 \pm 0.07$ & $11367 \pm 461$ & $\mathrm{c}$ & LIII \\
\hline CU Mon & DCEP & 0.6728 & 10.595 & 10.007 & 9.762 & -0.23 & -0.26 & $14.00 \pm 0.05$ & $13735 \pm 461$ & $b^{\dagger}$ & LIII \\
\hline EE Mon & DCEP & 0.6821 & 10.866 & 10.309 & 10.094 & -0.52 & -0.55 & $14.41 \pm 0.07$ & $14593 \pm 477$ & c & LIII \\
\hline FG Mon & DCEP & 0.6529 & 10.513 & 9.976 & 9.743 & -0.14 & -0.17 & $13.96 \pm 0.05$ & $13226 \pm 450$ & $b^{\dagger}$ & LIII \\
\hline FI Mon & DCEP & 0.5169 & 10.425 & 9.939 & 9.733 & -0.11 & -0.14 & $13.55 \pm 0.05$ & $12257 \pm 447$ & $b^{\dagger}$ & LIII \\
\hline V446 Mon & CEP & 0.2832 & 11.301 & 10.791 & 10.585 & -0.30 & -0.33 & $13.59 \pm 0.07$ & $12716 \pm 466$ & $\mathrm{c}$ & LIII \\
\hline V447 Mon & CEP & 0.3951 & 10.111 & 10.331 & 10.854 & -0.37 & -0.40 & $15.21 \pm 0.05$ & $18399 \pm 502$ & $b^{\dagger}$ & LIII \\
\hline V484 Mon & CEP & 0.4963 & 10.968 & 10.533 & 10.302 & -0.06 & -0.09 & $14.07 \pm 0.07$ & $13862 \pm 476$ & $\mathrm{c}$ & LIII \\
\hline V504 Mon & CEP & 0.4431 & 9.713 & 9.258 & 9.071 & 0.01 & -0.02 & $13.33 \pm 0.12$ & $12006 \pm 503$ & $\mathrm{c}$ & LIII \\
\hline V526 Mon & DCEPS & 0.4273 & 7.314 & 7.075 & 6.962 & -0.17 & -0.20 & $11.44 \pm 0.08$ & $9591 \pm 453$ & $\mathrm{c}^{*}$ & LIII \\
\hline V911 Mon & DCEP & 0.6971 & 9.481 & 8.932 & 8.694 & -0.01 & -0.04 & $13.05 \pm 0.07$ & $11822 \pm 464$ & $\mathrm{c}$ & LIII \\
\hline VZ Mon & DCEP & 0.7062 & 10.088 & 9.421 & 9.132 & -0.07 & -0.10 & $13.40 \pm 0.05$ & $12539 \pm 461$ & $\mathrm{~b}^{\dagger}$ & LIII \\
\hline YY Mon & DCEP & 0.5384 & 11.314 & 10.802 & 10.638 & -0.46 & -0.49 & $14.53 \pm 0.06$ & $15011 \pm 480$ & c & LIII \\
\hline R Mus & DCEP & 0.8757 & 4.904 & 4.577 & 4.465 & -0.08 & -0.11 & $9.66 \pm 0.05$ & $7523 \pm 450$ & $b^{\dagger}$ & LIII \\
\hline RT Mus & DCEP & 0.4894 & 7.181 & 6.809 & 6.660 & 0.12 & 0.09 & $10.50 \pm 0.05$ & $7464 \pm 447$ & b & LIII \\
\hline TZ Mus & DCEP & 0.6942 & 8.944 & 8.405 & 8.180 & 0.10 & 0.07 & $12.54 \pm 0.07$ & $7106 \pm 413$ & $\mathrm{c}$ & LIII \\
\hline BF Oph & DCEP & 0.6094 & 5.652 & 5.495 & 5.311 & 0.14 & 0.11 & $9.70 \pm 0.05$ & $7081 \pm 452$ & $\mathrm{~b}$ & LIII \\
\hline Y Oph & DCEPS & 1.2336 & 3.374 & 2.882 & 2.673 & 0.06 & 0.12 & $9.70 \pm 0.05$ & $7141 \pm 452$ & b & LII \\
\hline CR Ori & DCEP & 0.6911 & 9.705 & 9.294 & 9.114 & -0.19 & -0.22 & $13.59 \pm 0.06$ & $13023 \pm 474$ & $\mathrm{c}$ & LIII \\
\hline DF Ori & DCEP & 0.5025 & 10.618 & 10.090 & 9.834 & -0.28 & -0.31 & $13.54 \pm 0.07$ & $12868 \pm 473$ & $\mathrm{c}$ & LIII \\
\hline AS Per & DCEP & 0.6966 & 6.928 & 6.477 & 6.258 & 0.14 & 0.11 & $10.70 \pm 0.05$ & $9199 \pm 452$ & $\mathrm{a}$ & LIII \\
\hline AW Per & DCEP & 0.8105 & 5.213 & 4.832 & 4.657 & 0.04 & 0.01 & $9.56 \pm 0.05$ & $8731 \pm 452$ & $\mathrm{a}$ & LIII \\
\hline BM Per & DCEP & 1.3608 & 6.667 & 6.005 & 5.706 & 0.23 & 0.20 & $12.17 \pm 0.05$ & $10480 \pm 453$ & $\mathrm{a}$ & LIII \\
\hline CI Per & CEP: & 0.5286 & 10.666 & 10.427 & 10.293 & -0.32 & -0.35 & $14.38 \pm 0.07$ & $14135 \pm 465$ & $\mathrm{c}$ & LIII \\
\hline DW Per & DCEP & 0.5623 & 9.181 & 8.733 & 8.537 & -0.05 & -0.08 & $12.54 \pm 0.06$ & $10509 \pm 449$ & $\mathrm{c}$ & LIII \\
\hline GP Per & CEP & 0.3100 & 11.590 & 11.124 & 11.001 & -0.80 & -0.83 & $14.18 \pm 0.07$ & $14508 \pm 488$ & $\mathrm{c}$ & LIII \\
\hline HQ Per & CEP & 0.9364 & 9.179 & 8.626 & 8.465 & -0.35 & -0.38 & $13.67 \pm 0.06$ & $13198 \pm 475$ & $\mathrm{c}$ & LIII \\
\hline HZ Per & DCEP & 1.0523 & 9.149 & 8.339 & 8.002 & -0.25 & -0.28 & $13.30 \pm 0.05$ & $12274 \pm 459$ & $\mathrm{a}$ & LIII \\
\hline MM Per & DCEP & 0.6147 & 8.655 & 8.260 & 8.086 & -0.07 & -0.10 & $12.32 \pm 0.05$ & $10554 \pm 452$ & $\mathrm{a}$ & LIII \\
\hline OT Per & DCEP & 1.4165 & 8.735 & 7.914 & 7.565 & -0.07 & -0.10 & $14.07 \pm 0.05$ & $14178 \pm 470$ & $\mathrm{a}$ & LIII \\
\hline SV Per & DCEP & 1.0465 & 6.789 & 6.354 & 6.177 & 0.06 & 0.03 & $11.83 \pm 0.05$ & $10177 \pm 454$ & $\mathrm{a}$ & LIII \\
\hline SX Per & DCEP & 0.6325 & 8.757 & 8.347 & 8.167 & -0.03 & -0.06 & $12.44 \pm 0.05$ & $10851 \pm 455$ & $\mathrm{a}$ & LIII \\
\hline UX Per & DCEP & 0.6595 & 9.577 & 9.171 & 9.009 & -0.05 & -0.08 & $13.39 \pm 0.07$ & $11736 \pm 450$ & $\mathrm{c}$ & LIII \\
\hline UY Per & DCEP & 0.7296 & 8.079 & 7.536 & 7.334 & 0.18 & 0.15 & $11.82 \pm 0.06$ & $9737 \pm 449$ & $\mathrm{c}$ & LIII \\
\hline V440 Per & DCEPS & 0.8791 & 5.072 & 4.598 & 4.180 & -0.04 & 0.02 & $9.84 \pm 0.18$ & $8628 \pm 454$ & $c^{*}$ & LII \\
\hline V891 Per & DCEP & 0.5079 & 9.092 & 8.445 & 8.164 & 0.09 & 0.06 & $11.78 \pm 0.07$ & $9832 \pm 452$ & c & LIII \\
\hline VX Per & DCEP & 1.0370 & 6.879 & 6.439 & 6.240 & 0.06 & 0.03 & $11.84 \pm 0.05$ & $9678 \pm 447$ & $\mathrm{a}$ & LIII \\
\hline VY Per & DCEP & 0.7429 & 7.829 & 7.247 & 7.013 & 0.04 & 0.01 & $11.50 \pm 0.06$ & $9457 \pm 449$ & $\mathrm{c}$ & LIII \\
\hline NT Pup & DCEP & 1.1921 & 9.149 & 8.445 & 8.261 & -0.02 & -0.05 & $14.20 \pm 0.07$ & $11805 \pm 409$ & $\mathrm{c}$ & LIII \\
\hline V335 Pup & DCEPS & 0.6866 & 7.124 & 6.819 & 6.675 & 0.09 & 0.06 & $11.99 \pm 0.08$ & $9417 \pm 444$ & $\mathrm{c}^{*}$ & LIII \\
\hline RV Sco & DCEP & 0.7826 & 5.081 & 4.694 & 4.543 & 0.11 & 0.08 & $9.36 \pm 0.05$ & $7210 \pm 452$ & b & LIII \\
\hline V482 Sco & DCEP & 0.6559 & 5.976 & 5.591 & 5.438 & 0.20 & 0.17 & $9.83 \pm 0.05$ & $7021 \pm 452$ & $\mathrm{~b}$ & LIII \\
\hline V636 Sco & DCEP & 0.8323 & 4.908 & 4.537 & 4.398 & 0.10 & 0.07 & $9.40 \pm 0.05$ & $7219 \pm 452$ & $\mathrm{~b}^{\dagger}$ & LIII \\
\hline V950 Sco & DCEPS & 0.5289 & 5.729 & 5.425 & 5.295 & 0.21 & 0.18 & $10.07 \pm 0.05$ & $6933 \pm 452$ & $b^{\dagger}$ & LIII \\
\hline BX Sct & DCEP & 0.8069 & 7.834 & 7.147 & 6.774 & 0.28 & 0.25 & $11.32 \pm 0.07$ & $6395 \pm 450$ & c & LIII \\
\hline CK Sct & DCEP & 0.8701 & 7.381 & 6.819 & 6.591 & 0.21 & 0.18 & $11.52 \pm 0.05$ & $6196 \pm 449$ & $\mathrm{a}$ & LIII \\
\hline CM Sct & DCEP & 0.5930 & 8.300 & 7.818 & 7.558 & 0.15 & 0.12 & $11.60 \pm 0.07$ & $6153 \pm 450$ & $\mathrm{c}$ & LIII \\
\hline CN Sct & DCEP & 0.9997 & 7.842 & 7.069 & 6.697 & 0.33 & 0.30 & $11.82 \pm 0.05$ & $5996 \pm 446$ & $\mathrm{a}$ & LIII \\
\hline EW Sct & CEP(B) & 0.7652 & 4.314 & 3.726 & 3.466 & 0.04 & 0.10 & $8.01 \pm 0.05$ & $7580 \pm 452$ & $b^{\dagger}$ & LII \\
\hline SS Sct & DCEP & 0.5648 & 6.284 & 5.929 & 5.780 & 0.14 & 0.11 & $9.89 \pm 0.05$ & $7092 \pm 451$ & $\mathrm{a}$ & LIII \\
\hline TY Sct & DCEP & 1.0435 & 7.232 & 6.633 & 6.365 & 0.37 & 0.34 & $11.83 \pm 0.05$ & $5990 \pm 446$ & $\mathrm{a}$ & LIII \\
\hline V458 Sct & CEP(B) & 0.6848 & 7.173 & 6.697 & 6.562 & 0.09 & 0.08 & $10.99 \pm 0.20$ & $6488 \pm 468$ & $\mathrm{c}^{*}$ & SZI \\
\hline Y Sct & DCEP & 1.0146 & 6.460 & 5.894 & 5.627 & 0.23 & 0.20 & $11.02 \pm 0.05$ & $6510 \pm 451$ & $\mathrm{a}$ & LIII \\
\hline BQ Ser & CEP(B) & 0.6305 & 6.472 & 5.972 & 5.758 & -0.04 & 0.02 & $9.94 \pm 0.05$ & $7169 \pm 451$ & b & LII \\
\hline DV Ser & DCEP & 1.3629 & 8.595 & 7.843 & 7.471 & 0.47 & 0.44 & $13.84 \pm 0.07$ & $4053 \pm 321$ & $\mathrm{c}$ & LIII \\
\hline DG Sge & DCEP & 0.6471 & 9.135 & 8.526 & 8.275 & 0.13 & 0.10 & $12.41 \pm 0.06$ & $6711 \pm 420$ & $\mathrm{c}$ & LIII \\
\hline GX Sge & DCEP & 1.1106 & 8.274 & 7.575 & 7.276 & 0.29 & 0.26 & $12.87 \pm 0.05$ & $6521 \pm 399$ & $\mathrm{a}$ & LIII \\
\hline GY Sge & DCEP & 1.7081 & 5.545 & 4.841 & 4.512 & 0.29 & 0.26 & $12.10 \pm 0.05$ & $6779 \pm 429$ & $\mathrm{a}$ & LIII \\
\hline S Sge & DCEP & 0.9234 & 4.183 & 3.859 & 3.752 & 0.08 & 0.14 & $9.11 \pm 0.05$ & $7582 \pm 451$ & b & LII \\
\hline AP Sgr & DCEP & 0.7040 & 5.347 & 5.012 & 4.883 & 0.10 & 0.16 & $9.49 \pm 0.05$ & $7160 \pm 452$ & b & LII \\
\hline BB Sgr & DCEP & 0.8220 & 5.044 & 4.653 & 4.500 & 0.08 & 0.14 & $9.44 \pm 0.05$ & $7203 \pm 452$ & b & LII \\
\hline U Sgr & DCEP & 0.8290 & 4.506 & 4.100 & 3.912 & 0.08 & 0.14 & $8.85 \pm 0.05$ & $7371 \pm 452$ & $\mathrm{a}$ & LII \\
\hline V350 Sgr & DCEP & 0.7122 & 5.641 & 5.279 & 5.133 & 0.18 & 0.24 & $9.73 \pm 0.05$ & $7092 \pm 452$ & b & LII \\
\hline W Sgr & DCEP & 0.8805 & 3.243 & 2.923 & 2.814 & 0.02 & 0.08 & $8.03 \pm 0.05$ & $7536 \pm 452$ & b & LII \\
\hline Y Sgr & DCEP & 0.7614 & 4.067 & 3.717 & 3.577 & 0.05 & 0.11 & $8.36 \pm 0.05$ & $7483 \pm 452$ & b & LII \\
\hline YZ Sgr & DCEP & 0.9802 & 5.364 & 5.000 & 4.840 & 0.06 & 0.12 & $10.33 \pm 0.05$ & $6848 \pm 452$ & $\mathrm{a}$ & LII \\
\hline AE Tau & CEP & 0.5907 & 9.442 & 8.949 & 8.750 & -0.18 & -0.21 & $12.81 \pm 0.07$ & $11584 \pm 465$ & $\mathrm{c}$ & LIII \\
\hline EF Tau & DCEP & 0.5376 & 10.876 & 10.402 & 10.249 & -0.74 & -0.68 & $14.17 \pm 0.07$ & $14703 \pm 495$ & $\mathrm{c}$ & LII \\
\hline LR TrA & DCEPS & 0.3852 & 6.307 & 6.020 & 5.880 & 0.31 & 0.28 & $10.16 \pm 0.07$ & $7176 \pm 450$ & $c^{*}$ & LIII \\
\hline $\mathrm{R} \operatorname{Tr} \mathrm{A}$ & DCEP & 0.5301 & 5.257 & 4.946 & 4.831 & 0.19 & 0.16 & $8.87 \pm 0.05$ & $7519 \pm 451$ & $\mathrm{~b}$ & LIII \\
\hline $\mathrm{S} \operatorname{Tr} \mathrm{A}$ & DCEP & 0.8010 & 5.021 & 4.696 & 4.581 & 0.21 & 0.18 & $9.53 \pm 0.05$ & $7328 \pm 451$ & b & LIII \\
\hline $\mathrm{U} \operatorname{Tr} \mathrm{A}$ & CEP(B) & 0.4097 & 6.542 & 6.351 & 6.286 & -0.15 & -0.16 & $9.89 \pm 0.19$ & $7207 \pm 455$ & $\mathrm{c}^{*}$ & SZI \\
\hline AE Vel & DCEP & 0.8533 & 7.589 & 7.096 & 6.867 & 0.14 & 0.11 & $11.80 \pm 0.07$ & $8026 \pm 433$ & $\mathrm{c}$ & LIII \\
\hline AP Vel & CEP(B) & 0.4952 & 7.730 & 7.351 & 7.188 & -0.06 & -0.07 & $11.03 \pm 0.19$ & $8291 \pm 446$ & $c^{*}$ & SZI \\
\hline CS Vel & DCEP & 0.7712 & 8.771 & 8.246 & 8.011 & 0.12 & 0.09 & $12.63 \pm 0.05$ & $8231 \pm 414$ & $\mathrm{~b}$ & LIII \\
\hline CX Vel & DCEP & 0.7963 & 8.309 & 7.769 & 7.517 & 0.16 & 0.13 & $12.21 \pm 0.07$ & $8296 \pm 427$ & c & LIII \\
\hline DK Vel & DCEP & 0.3947 & 8.820 & 8.496 & 8.386 & 0.16 & 0.13 & $12.67 \pm 0.12$ & $8421 \pm 418$ & $\mathrm{c}$ & LIII \\
\hline EX Vel & DCEP & 1.1217 & 8.365 & 7.733 & 7.553 & 0.07 & 0.04 & $13.30 \pm 0.07$ & $8871 \pm 393$ & $\mathrm{c}$ & LIII \\
\hline FG Vel & DCEP & 0.8098 & 8.509 & 7.961 & 7.719 & 0.02 & -0.01 & $12.45 \pm 0.07$ & $8239 \pm 420$ & $\mathrm{c}$ & LIII \\
\hline FN Vel & CEP & 0.7263 & 7.686 & 7.264 & 7.067 & 0.15 & 0.12 & $11.64 \pm 0.07$ & $7891 \pm 435$ & $\mathrm{c}$ & LIII \\
\hline
\end{tabular}


A\&A 566, A37 (2014)

Table 4. continued.

\begin{tabular}{|c|c|c|c|c|c|c|c|c|c|c|c|}
\hline Name & Type & $\begin{array}{l}\log (P) \\
\text { [days] }\end{array}$ & $\begin{array}{c}\langle J\rangle \\
\text { mag }\end{array}$ & $\begin{array}{l}\langle H\rangle \\
\mathrm{mag}\end{array}$ & $\begin{array}{c}\langle K\rangle \\
\mathrm{mag}\end{array}$ & {$[\mathrm{Fe} / \mathrm{H}]_{\text {lit }}$} & {$[\mathrm{Fe} / \mathrm{H}]$} & $\begin{array}{c}\mu^{d} \\
\mathrm{mag}\end{array}$ & $\begin{array}{l}R_{\mathrm{G}}^{\mathrm{e}} \\
(\mathrm{pc})\end{array}$ & Notes & $\operatorname{Ref}^{f}$ \\
\hline SV Vel & DCEP & 1.1491 & 6.370 & 5.944 & 5.769 & 0.12 & 0.09 & $11.77 \pm 0.05$ & $7632 \pm 433$ & $\mathrm{~b}^{\dagger}$ & LIII \\
\hline XX Vel & DCEP & 0.8441 & 8.246 & 7.767 & 7.574 & 0.11 & 0.08 & $12.51 \pm 0.07$ & $7760 \pm 415$ & $\mathrm{c}$ & LIII \\
\hline AS Vul & DCEP & 1.0872 & 8.383 & 7.728 & 7.454 & 0.22 & 0.19 & $13.02 \pm 0.05$ & $7150 \pm 390$ & $\mathrm{a}$ & LIII \\
\hline DG Vul & CEP & 1.1339 & 7.331 & 6.642 & 6.373 & 0.19 & 0.16 & $12.08 \pm 0.07$ & $7233 \pm 427$ & $\mathrm{c}$ & LIII \\
\hline S Vul & DCEP & 1.8355 & 5.415 & 4.819 & 4.551 & 0.12 & 0.09 & $12.68 \pm 0.05$ & $7103 \pm 407$ & a & LIII \\
\hline SV Vul & DCEP & 1.6533 & 4.547 & 4.063 & 3.868 & 0.05 & 0.11 & $11.51 \pm 0.05$ & $7284 \pm 438$ & $\mathrm{a}$ & LII \\
\hline T Vul & DCEP & 0.6469 & 4.558 & 4.273 & 4.185 & 0.01 & 0.07 & $8.66 \pm 0.05$ & $7793 \pm 451$ & $\mathrm{~b}$ & LII \\
\hline U Vul & DCEP & 0.9026 & 4.506 & 4.086 & 3.891 & 0.19 & 0.16 & $9.06 \pm 0.05$ & $7597 \pm 451$ & $\mathrm{a}$ & LIII \\
\hline X Vul & DCEP & 0.8007 & 5.912 & 5.424 & 5.194 & 0.07 & 0.13 & $9.95 \pm 0.05$ & $7560 \pm 449$ & $\mathrm{a}$ & LII \\
\hline $\mathrm{U}$ Car & DCEP & 1.5885 & 4.133 & 3.683 & 3.510 & 0.17 & 0.25 & $10.98 \pm 0.05$ & $7574 \pm 443$ & $\mathrm{~b}$ & ROM \\
\hline WZ Car & DCEP & 1.3620 & 6.946 & 6.470 & 6.279 & -0.02 & 0.06 & $12.95 \pm 0.05$ & $7601 \pm 396$ & $\mathrm{~b}$ & ROM \\
\hline V Cen & DCEP & 0.7399 & 5.019 & 4.642 & 4.498 & 0.04 & 0.12 & $9.18 \pm 0.05$ & $7459 \pm 451$ & $\mathrm{~b}$ & ROM \\
\hline XX Cen & DCEP & 1.0396 & 5.937 & 5.544 & 5.396 & 0.04 & 0.12 & $11.07 \pm 0.05$ & $7015 \pm 445$ & $\mathrm{~b}$ & ROM \\
\hline$\beta$ Dor & DCEP & 0.9931 & 2.387 & 2.043 & 1.943 & -0.14 & -0.06 & $7.53 \pm 0.05$ & $7936 \pm 451$ & $\mathrm{~b}$ & ROM \\
\hline$\zeta \mathrm{Gem}$ & DCEP & 1.0065 & 2.561 & 2.215 & 2.114 & -0.19 & -0.11 & $7.74 \pm 0.05$ & $8273 \pm 452$ & $\mathrm{~b}$ & ROM \\
\hline GH Lup & CEP & 0.9678 & 5.432 & 4.973 & 4.801 & 0.05 & 0.13 & $10.17 \pm 0.05$ & $7082 \pm 450$ & $\mathrm{~b}$ & ROM \\
\hline T Mon & DCEP & 1.4318 & 4.068 & 3.622 & 3.459 & -0.04 & 0.04 & $10.41 \pm 0.05$ & $9056 \pm 452$ & $\mathrm{a}$ & ROM \\
\hline S Mus & DCEP & 0.9850 & 4.500 & 4.139 & 4.005 & 0.13 & 0.21 & $9.53 \pm 0.05$ & $7576 \pm 450$ & $\mathrm{~b}$ & ROM \\
\hline UU Mus & DCEP & 1.0658 & 7.469 & 7.004 & 6.818 & 0.11 & 0.19 & $12.51 \pm 0.05$ & $7097 \pm 414$ & $\mathrm{~b}$ & ROM \\
\hline S Nor & DCEP & 0.9892 & 4.674 & 4.288 & 4.149 & 0.02 & 0.10 & $9.67 \pm 0.05$ & $7232 \pm 451$ & $\mathrm{~b}$ & ROM \\
\hline U Nor & DCEP & 1.1019 & 5.858 & 5.251 & 4.979 & 0.07 & 0.15 & $10.63 \pm 0.05$ & $6876 \pm 450$ & $\mathrm{~b}$ & ROM \\
\hline EU Tau & DCEPS & 0.3227 & 6.691 & 6.360 & 6.254 & 0.04 & 0.12 & $10.30 \pm 0.09$ & $9073 \pm 454$ & $\mathrm{c}^{*}$ & ROM \\
\hline SZ Tau & DCEPS & 0.4981 & 4.779 & 4.422 & 4.298 & 0.07 & 0.15 & $8.93 \pm 0.05$ & $8518 \pm 452$ & $\mathrm{~b}$ & ROM \\
\hline AO Aur & DCEP & 0.8301 & 8.631 & 8.200 & 8.027 & -0.41 & -0.27 & $12.96 \pm 0.05$ & $11835 \pm 461$ & $\mathrm{a}$ & LEM \\
\hline AX Aur & DCEP & 0.4838 & 9.929 & 9.491 & 9.283 & -0.22 & -0.08 & $13.02 \pm 0.05$ & $11955 \pm 461$ & $\mathrm{a}$ & LEM \\
\hline BK Aur & DCEP & 0.9032 & 7.291 & 6.885 & 6.711 & -0.07 & 0.07 & $11.90 \pm 0.05$ & $10207 \pm 453$ & $\mathrm{a}$ & LEM \\
\hline SY Aur & DCEP & 1.0062 & 6.913 & 6.525 & 6.348 & -0.07 & 0.07 & $11.90 \pm 0.05$ & $10271 \pm 454$ & $\mathrm{a}$ & LEM \\
\hline Y Aur & DCEP & 0.5865 & 7.651 & 7.287 & 7.113 & -0.26 & -0.12 & $11.27 \pm 0.05$ & $9692 \pm 453$ & $\mathrm{a}$ & LEM \\
\hline YZ Aur & DCEP & 1.2599 & 7.487 & 6.905 & 6.669 & -0.33 & -0.19 & $12.89 \pm 0.05$ & $11668 \pm 459$ & $\mathrm{a}$ & LEM \\
\hline AQ Car & DCEP & 0.9898 & 7.138 & 6.757 & 6.618 & -0.30 & -0.16 & $12.14 \pm 0.05$ & $7658 \pm 425$ & $\mathrm{~b}$ & LEM \\
\hline $1 \mathrm{Car}$ & DCEP & 1.5507 & 1.706 & 1.225 & 1.073 & 0.10 & 0.24 & $8.40 \pm 0.05$ & $7845 \pm 451$ & $\mathrm{~b}$ & LEM \\
\hline UX Car & DCEP & 0.5661 & 6.992 & 6.728 & 6.620 & -0.10 & 0.04 & $10.82 \pm 0.05$ & $7698 \pm 444$ & $\mathrm{~b}$ & LEM \\
\hline V Car & DCEP & 0.8259 & 5.753 & 5.402 & 5.274 & -0.06 & 0.08 & $10.27 \pm 0.05$ & $7915 \pm 447$ & $\mathrm{~b}$ & LEM \\
\hline V397 Car & DCEPS & 0.3145 & 6.857 & 6.533 & 6.404 & -0.08 & 0.06 & $10.42 \pm 0.07$ & $7678 \pm 447$ & $\mathrm{c}^{*}$ & LEM \\
\hline VY Car & DCEP & 1.2785 & 5.404 & 4.958 & 4.792 & -0.06 & 0.08 & $11.22 \pm 0.05$ & $7627 \pm 441$ & $\mathrm{~b}$ & LEM \\
\hline RY Cma & DCEP & 0.6701 & 6.363 & 6.042 & 5.874 & 0.00 & 0.14 & $10.35 \pm 0.05$ & $8796 \pm 450$ & $\mathrm{a}$ & LEM \\
\hline RZ Cma & DCEP & 0.6289 & 7.533 & 7.178 & 6.967 & -0.20 & -0.06 & $11.25 \pm 0.05$ & $9162 \pm 448$ & $\mathrm{a}$ & LEM \\
\hline BV Mon & DCEP & 0.4792 & 9.005 & 8.584 & 8.420 & -0.10 & 0.04 & $12.18 \pm 0.05$ & $10398 \pm 452$ & $\mathrm{a}$ & LEM \\
\hline EK Mon & DCEP & 0.5975 & 8.516 & 8.051 & 7.792 & -0.05 & 0.09 & $11.86 \pm 0.07$ & $9960 \pm 453$ & $\mathrm{c}$ & LEM \\
\hline UY Mon & DCEPS & 0.3799 & 8.175 & 7.958 & 7.897 & -0.33 & -0.19 & $12.26 \pm 0.10$ & $10553 \pm 464$ & $\mathrm{c}^{*}$ & LEM \\
\hline WW Mon & DCEP & 0.6686 & 9.963 & 9.507 & 9.333 & -0.32 & -0.18 & $13.70 \pm 0.05$ & $13176 \pm 463$ & $\mathrm{a}^{\dagger}$ & LEM \\
\hline GQ Ori & DCEP & 0.9353 & 7.068 & 6.650 & 6.503 & 0.11 & 0.25 & $11.81 \pm 0.05$ & $10129 \pm 453$ & $\mathrm{~b}$ & LEM \\
\hline AD Pup & DCEP & 1.1333 & 7.723 & 7.341 & 7.144 & -0.20 & -0.06 & $13.12 \pm 0.07$ & $10588 \pm 434$ & $\mathrm{c}$ & LEM \\
\hline AP Pup & DCEP & 0.7062 & 5.788 & 5.443 & 5.322 & -0.16 & -0.02 & $9.93 \pm 0.05$ & $8234 \pm 449$ & $\mathrm{~b}^{\dagger}$ & LEM \\
\hline AT Pup & DCEP & 0.8238 & 6.400 & 6.054 & 5.930 & -0.22 & -0.08 & $10.93 \pm 0.05$ & $8484 \pm 445$ & $\mathrm{~b}$ & LEM \\
\hline MY Pup & DCEPS & 0.7555 & 4.333 & 4.050 & 3.950 & -0.25 & -0.11 & $9.54 \pm 0.05$ & $8096 \pm 450$ & $\mathrm{~b}^{\dagger}$ & LEM \\
\hline RS Pup & DCEP & 1.6169 & 4.365 & 3.828 & 3.619 & 0.07 & 0.21 & $11.09 \pm 0.05$ & $8585 \pm 444$ & $\mathrm{~b}$ & LEM \\
\hline VX Pup & $\mathrm{CEP}(\mathrm{B})$ & 0.4787 & 7.085 & 6.760 & 6.667 & -0.15 & -0.01 & $10.54 \pm 0.17$ & $8703 \pm 453$ & $\mathrm{c}^{*}$ & LEM \\
\hline WX Pup & DCEP & 0.9512 & 7.134 & 6.721 & 6.572 & -0.15 & -0.01 & $11.94 \pm 0.05$ & $9351 \pm 441$ & $\mathrm{~b}$ & LEM \\
\hline AV Tau & DCEP & 0.5582 & 9.196 & 8.648 & 8.405 & -0.17 & -0.03 & $12.29 \pm 0.05$ & $10809 \pm 457$ & $\mathrm{a}$ & LEM \\
\hline ST Tau & DCEP & 0.6058 & 6.269 & 5.904 & 5.746 & -0.14 & 0.00 & $9.98 \pm 0.05$ & $8897 \pm 452$ & $\mathrm{a}$ & LEM \\
\hline AH Vel & DCEPS & 0.6260 & 4.568 & 4.327 & 4.235 & -0.05 & 0.09 & $9.41 \pm 0.05$ & $8074 \pm 450$ & $\mathrm{~b}^{\dagger}$ & LEM \\
\hline AX Vel & $\mathrm{CEP}(\mathrm{B})$ & 0.4138 & 6.773 & 6.478 & 6.366 & -0.15 & -0.01 & $10.03 \pm 0.05$ & $8120 \pm 448$ & $\mathrm{~b}$ & LEM \\
\hline BG Vel & DCEP & 0.8403 & 5.410 & 4.972 & 4.797 & -0.10 & 0.04 & $10.52 \pm 0.05$ & $8000 \pm 446$ & $\mathrm{~b}^{\dagger}$ & LEM \\
\hline DR Vel & DCEP & 1.0492 & 6.583 & 6.035 & 5.811 & -0.01 & 0.13 & $11.36 \pm 0.05$ & $8054 \pm 439$ & $\mathrm{~b}$ & LEM \\
\hline RY Vel & DCEP & 1.4493 & 5.638 & 5.136 & 4.920 & -0.05 & 0.09 & $11.85 \pm 0.05$ & $7774 \pm 432$ & $\mathrm{~b}$ & LEM \\
\hline RZ Vel & DCEP & 1.3096 & 4.919 & 4.474 & 4.298 & 0.05 & 0.19 & $10.83 \pm 0.05$ & $8249 \pm 445$ & $\mathrm{~b}$ & LEM \\
\hline ST Vel & DCEP & 0.7678 & 7.244 & 6.734 & 6.538 & -0.14 & 0.00 & $11.19 \pm 0.07$ & $8158 \pm 442$ & $\mathrm{c}$ & LEM \\
\hline SW Vel & DCEP & 1.3700 & 5.873 & 5.407 & 5.222 & -0.15 & -0.01 & $11.93 \pm 0.05$ & $8457 \pm 433$ & $\mathrm{~b}$ & LEM \\
\hline SX Vel & DCEP & 0.9800 & 6.500 & 6.133 & 5.991 & -0.18 & -0.04 & $11.49 \pm 0.05$ & $8334 \pm 439$ & $\mathrm{~b}$ & LEM \\
\hline $\mathrm{T}$ Vel & DCEP & 0.6665 & 6.170 & 5.782 & 5.632 & -0.02 & 0.12 & $10.06 \pm 0.05$ & $8084 \pm 448$ & $\mathrm{~b}$ & LEM \\
\hline V Vel & DCEP & 0.6406 & 6.021 & 5.687 & 5.567 & -0.30 & -0.16 & $9.96 \pm 0.05$ & $7888 \pm 448$ & $\mathrm{~b}$ & LEM \\
\hline
\end{tabular}


K. Genovali et al.: The fine structure of the metallicity gradient

Table 5. Individual ages, distances, and metallicities for the current sample of open clusters.

\begin{tabular}{|c|c|c|c|c|c|c|}
\hline ID & $\begin{array}{r}\text { Age } \\
\text { (Gyrs) } \\
\end{array}$ & $\begin{array}{r}R_{\mathrm{G}} \\
(\mathrm{kpc})\end{array}$ & {$[\mathrm{Fe} / \mathrm{H}]_{\text {lit }}$} & {$[\mathrm{Fe} / \mathrm{H}]_{\mathrm{rsc}}$} & Ref. met. & Ref. age-dist. \\
\hline Be 17 & 10.06 & 10.3 & -0.15 & -0.13 & Friel et al. (2005) & $\mathrm{SF}^{a}$ \\
\hline Be 18 & 5.69 & 11.5 & -0.44 & -0.44 & Yong et al. (2012) & SF \\
\hline Be 20 & 4.05 & 15.4 & -0.30 & -0.31 & Sestito et al. (2008) & SF \\
\hline Be 21 & 2.18 & 13.7 & -0.31 & -0.31 & Yong et al. (2012) & SF \\
\hline Be 22 & 4.26 & 11.4 & -0.45 & -0.45 & Yong et al. (2012) & SF \\
\hline Be 25 & 4.00 & 17.6 & -0.20 & -0.22 & Carraro et al. (2007b) & $\mathrm{C}^{b}$ \\
\hline Be 29 & 4.34 & 18.1 & -0.31 & -0.32 & Sestito et al. (2008) & SF \\
\hline Be 31 & 5.32 & 11.5 & -0.53 & -0.49 & Friel et al. (2005) & $\mathrm{SF}$ \\
\hline Be 32 & 5.91 & 10.7 & -0.38 & -0.38 & Yong et al. (2012) & SF \\
\hline Be 39 & 7.00 & 11.1 & 0.03 & 0.05 & Friel et al. (2010) & SF \\
\hline Be 66 & 3.98 & 12.0 & -0.48 & -0.46 & Villanova et al. (2005) & SF \\
\hline Be 73 & 1.50 & 16.7 & -0.22 & -0.24 & Carraro et al. (2007b) & $\mathrm{C}$ \\
\hline Be 75 & 3.00 & 15.6 & -0.22 & -0.24 & Carraro et al. (2007b) & $\mathrm{C}$ \\
\hline Blancol & 0.1 & 7.9 & 0.4 & -0.02 & Ford et al. (2005) & W \\
\hline Cr 110 & 1.70 & 9.7 & 0.03 & 0.03 & Pancino et al. (2010) & $\mathrm{B}^{c}$ \\
\hline Cr 261 & 6.00 & 6.9 & 0.13 & 0.12 & Sestito et al. (2008) & B \\
\hline Hyades & 0.70 & 8.0 & 0.11 & 0.11 & Carrera et al. (2011) & SF \\
\hline IC 166 & 2.00 & 10.2 & -0.32 & -0.32 & Friel \& Janes (1993) & FJ \\
\hline IC 4651 & 1.68 & 7.1 & 0.11 & 0.15 & Carretta et al. (2004) & SF \\
\hline King 8 & 0.80 & 11.3 & -0.40 & -0.40 & Friel \& Janes (1993) & FJ \\
\hline M 67 & 4.30 & 8.5 & -0.01 & 0.01 & Jacobson et al. (2011b) & SF \\
\hline Mel 66 & 5.33 & 8.9 & -0.33 & -0.34 & Sestito et al. (2008) & SF \\
\hline Mel 71 & 0.2 & 10.3 & -0.32 & -0.10 & Brown et al. (1996) & W \\
\hline NGC 1193 & 4.23 & 11.4 & -0.22 & -0.20 & Friel et al. (2010) & SF \\
\hline NGC 1245 & 1.06 & 10.5 & -0.04 & -0.02 & Jacobson et al. (2011b) & $\mathrm{SF}$ \\
\hline NGC 1817 & 1.12 & 9.7 & -0.16 & -0.14 & Jacobson et al. (2011b) & SF \\
\hline NGC 188 & 6.30 & 8.8 & -0.03 & -0.01 & Jacobson et al. (2011b) & SF \\
\hline NGC 1883 & 0.65 & 11.7 & -0.01 & 0.01 & Jacobson et al. (2009) & $\mathrm{C}$ \\
\hline NGC 1901 & 0.8 & 7.9 & -0.08 & -0.08 & Carraro et al. (2007b) & $\mathrm{W}$ \\
\hline NGC 2099 & 0.43 & 9.2 & 0.01 & 0.01 & Pancino et al. (2010) & B \\
\hline NGC 2112 & 2.50 & 8.6 & -0.52 & -0.52 & Friel \& Janes (1993) & FJ \\
\hline NGC 2141 & 2.45 & 12.0 & 0.00 & 0.02 & Jacobson et al. (2009) & SF \\
\hline NGC 2158 & 2.00 & 11.5 & -0.28 & -0.26 & Jacobson et al. (2011b) & $\mathrm{C}$ \\
\hline NGC 2194 & 0.87 & 10.5 & -0.08 & -0.06 & Jacobson et al. (2011b) & SF \\
\hline NGC 2204 & 2.00 & 11.2 & -0.23 & -0.21 & Jacobson et al. (2011a) & SF \\
\hline NGC 2243 & 4.80 & 10.1 & -0.42 & -0.40 & Jacobson et al. (2011a) & B \\
\hline NGC 2324 & 0.67 & 10.7 & -0.17 & -0.18 & Bragaglia et al. (2008) & SF \\
\hline NGC 2355 & 0.79 & 10.0 & -0.08 & -0.06 & Jacobson et al. (2011b) & SF \\
\hline NGC 2360 & 1.90 & 8.8 & -0.28 & -0.28 & Friel \& Janes (1993) & FJ \\
\hline NGC 2420 & 2.20 & 10.0 & -0.20 & -0.18 & Jacobson et al. (2011b) & SF \\
\hline NGC 2425 & 3.55 & 9.5 & -0.15 & -0.13 & Jacobson et al. (2011b) & $\mathrm{C}$ \\
\hline NGC 2477 & 1.00 & 8.3 & 0.07 & 0.06 & Bragaglia et al. (2008) & SF \\
\hline NGC 2506 & 1.70 & 10.3 & -0.20 & -0.16 & Carretta et al. (2004) & B \\
\hline NGC 2539 & 0.4 & 8.8 & 0.13 & 0.13 & Santos et al. (2009) & $\mathrm{W}$ \\
\hline NGC 2660 & 0.73 & 8.5 & 0.04 & 0.03 & Bragaglia et al. (2008) & SF \\
\hline NGC 2682 & 5.00 & 8.5 & -0.09 & -0.09 & Friel \& Janes (1993) & $\mathrm{FJ}$ \\
\hline NGC 3680 & 4.00 & 7.8 & -0.16 & -0.16 & Friel \& Janes (1993) & FJ \\
\hline NGC 3960 & 0.90 & 7.3 & -0.12 & -0.08 & Bragaglia et al. (2006) & B \\
\hline NGC 5822 & 1.20 & 7.4 & -0.21 & -0.21 & Friel \& Janes (1993) & FJ \\
\hline NGC 6134 & 0.93 & 7.1 & 0.15 & 0.19 & Carretta et al. (2004) & $\mathrm{W}^{d}$ \\
\hline NGC 6192 & 0.13 & 6.5 & 0.12 & 0.10 & Magrini et al. (2010) & $\mathrm{W}$ \\
\hline NGC 6253 & 3.00 & 6.5 & 0.36 & 0.35 & Sestito et al. (2008) & B \\
\hline NGC 6404 & 0.50 & 6.1 & 0.11 & 0.09 & Magrini et al. (2010) & $\mathrm{C}$ \\
\hline NGC 6475 & 0.20 & 7.6 & 0.03 & 0.03 & Villanova et al. (2009) & $\mathrm{C}$ \\
\hline NGC 6583 & 1.00 & 5.9 & 0.37 & 0.35 & Magrini et al. (2010) & $\mathrm{C}$ \\
\hline NGC 6791 & 8.00 & 7.6 & 0.42 & 0.42 & Geisler et al. (2012) & $\mathrm{C}$ \\
\hline NGC 6819 & 2.00 & 7.6 & 0.09 & 0.11 & Bragaglia et al. (2001) & B \\
\hline NGC 6939 & 2.2 & 8.1 & 0.00 & 0.00 & Jacobson et al. (2007) & W \\
\hline NGC 7142 & 4.04 & 9.2 & 0.13 & 0.15 & Jacobson et al. (2008) & SF \\
\hline NGC 752 & 1.24 & 8.2 & 0.08 & 0.08 & Carrera et al. (2011) & SF \\
\hline NGC 7789 & 1.80 & 8.9 & 0.02 & 0.04 & Jacobson et al. (2011b) & SF \\
\hline Praesepe & 0.70 & 8.1 & 0.16 & 0.16 & Carrera et al. (2011) & SF \\
\hline PWM4 & 7.00 & 18.4 & -0.34 & -0.34 & Yong et al. (2012) & $\mathrm{YON}^{e}$ \\
\hline Ruprecht 4 & 0.80 & 12.0 & -0.09 & -0.11 & Carraro et al. (2007b) & $\mathrm{C}$ \\
\hline Ruprecht 7 & 0.80 & 13.3 & -0.26 & -0.28 & Carraro et al. (2007b) & $\mathrm{C}$ \\
\hline Saurer 1 & 5.00 & 20.1 & -0.38 & -0.38 & Carraro et al. (2004) & $\mathrm{C}$ \\
\hline To 2 & 2.50 & 14.9 & -0.60 & -0.60 & Friel \& Janes (1993) & FJ \\
\hline
\end{tabular}

Notes. From left to right the columns give the cluster ID, the Galactocentric distance, the iron abundance available in the literature, the metallicity rescaled to current solar abundance, the references for the metallicity and the references for ages and distances. The acronyms given in Col. 7 refers to SF: (Salaris et al. 2004; Friel 1995), C (Carraro group): (Carraro et al. 2002, 2005a,b,c; Moitinho et al. 2006; Villanova et al. 2007, 2009), B (BOCCE): (Gratton \& Contarini 1994; Bragaglia et al. 2001; Kalirai \& Tosi 2004; Bragaglia et al. 2006; Carretta et al. 2004, 2005, 2007), W: (WEBDA), and YON: (Yong et al. 2012). 
A\&A 566, A37 (2014)

Table 6. Structural parameters and metallicities of the candidate Cepheid groups.

\begin{tabular}{|c|c|c|c|c|c|c|c|c|c|c|c|c|c|}
\hline ID & $\begin{array}{c}x \\
(\mathrm{kpc}) \\
\end{array}$ & $\begin{array}{c}y \\
(\mathrm{kpc})\end{array}$ & $\begin{array}{c}z \\
(\mathrm{kpc})\end{array}$ & $\begin{array}{c}R_{\mathrm{G}} \\
(\mathrm{kpc})\end{array}$ & $\begin{array}{l}\delta R_{\mathrm{G}}^{-} \\
(\mathrm{kpc})\end{array}$ & $\begin{array}{l}\delta R_{\mathrm{G}}^{+} \\
(\mathrm{kpc})\end{array}$ & $\begin{array}{c}D \\
(\mathrm{kpc})\end{array}$ & $N_{s}$ & $\begin{array}{c}P \\
\text { (days) } \\
\end{array}$ & $\begin{array}{c}\sigma(P) \\
\text { (days) }\end{array}$ & $\begin{array}{r}\bar{\rho}_{\mathrm{CG}} \\
\left(\mathrm{kpc}^{-3}\right)\end{array}$ & $\Delta[\mathrm{Fe} / \mathrm{H}]$ & $\sigma(\Delta[\mathrm{Fe} / \mathrm{H}])$ \\
\hline I & 0.766 & -1.612 & -0.033 & 6.374 & 0.384 & 0.475 & 1.213 & 20 & 8.69 & 5.17 & 16.8 & -0.039 & 0.010 \\
\hline II & -0.939 & -1.562 & -0.035 & 6.447 & 0.286 & 0.430 & 0.827 & 7 & 8.69 & 3.78 & 4.8 & -0.003 & 0.004 \\
\hline III & -1.636 & -0.655 & -0.003 & 7.466 & 0.775 & 0.560 & 1.661 & 37 & 7.48 & 7.35 & 37.7 & -0.063 & 0.010 \\
\hline IV & 0.016 & -0.503 & -0.034 & 7.437 & 0.503 & 0.501 & 1.879 & 52 & 6.91 & 5.20 & 45.7 & -0.009 & 0.008 \\
\hline $\mathrm{V}$ & 2.182 & -0.137 & 0.044 & 8.103 & 0.464 & 0.371 & 0.847 & 7 & 8.74 & 5.84 & 8.8 & 0.009 & 0.002 \\
\hline VI & 1.549 & 0.918 & -0.099 & 8.993 & 0.133 & 0.186 & 0.497 & 6 & 5.30 & 1.34 & 4.2 & 0.033 & 0.003 \\
\hline VII & 2.608 & 1.167 & 0.050 & 9.473 & 0.396 & 0.190 & 0.654 & 11 & 6.72 & 3.51 & 4.7 & 0.062 & 0.007 \\
\hline VIII & 1.732 & 1.515 & 0.014 & 9.613 & 0.219 & 0.261 & 0.641 & 11 & 5.08 & 2.37 & 4.2 & 0.040 & 0.009 \\
\hline IX & 2.511 & 1.777 & -0.008 & 10.036 & 0.144 & 0.226 & 0.621 & 7 & 5.38 & 0.79 & 4.4 & 0.046 & 0.006 \\
\hline $\mathrm{X}$ & 3.269 & 1.754 & -0.047 & 10.231 & 0.187 & 0.230 & 0.449 & 6 & 5.63 & 1.60 & 6.8 & 0.033 & 0.007 \\
\hline
\end{tabular}

Notes. Structural parameters of the ten candidate Cepheid groups identified in Sect. 4. From left to right Col. 1 gives the identification number, while Cols 2-4 give the Galactic coordinates $(x, y, z)$ of the barycenter. Columns 5-7 give the Galactocentric radius of the barycenter together with the Galactocentric distance of both the inner and the outer edge of the Cepheid group. Column 8 gives the diameter of the CG, while Col. 9 lists the number of Cepheid per CG. Columns 10 and 11 give the mean period and its intrinsic dispersion, while Col. 12 gives the density. Columns 13 and 14 give the mean residual iron abundance and its intrinsic dispersion. 
Table 7. Intrinsic parameters for field and cluster (NGC 1850, NGC 1866) Magellanic Cepheids with accurate iron abundances.

\begin{tabular}{|c|c|c|c|c|c|}
\hline ID & $\begin{array}{l}\text { Period } \\
\text { (days) }\end{array}$ & {$[\mathrm{Fe} / \mathrm{H}]$} & Ref. & $\begin{array}{r}A_{V} \\
\mathrm{mag}\end{array}$ & Ref. \\
\hline \multicolumn{6}{|c|}{ SMC } \\
\hline HV 817 & 18.9234 & -0.83 & Romaniello et al. (2008) & 0.92 & Caldwell et al. $(1986,2001)$ \\
\hline HV 823 & 31.9154 & -0.81 & Romaniello et al. (2008) & 0.94 & Soszynski et al. (2008); Soszyñski et al. (2010) \\
\hline HV 824 & 65.7658 & -0.74 & Romaniello et al. (2008) & 0.99 & Soszynski et al. (2008); Soszyñski et al. (2010) \\
\hline HV 829 & 85.3100 & -0.75 & Romaniello et al. (2008) & 0.76 & Soszynski et al. (2008); Soszyñski et al. (2010) \\
\hline HV 834 & 73.4514 & -0.64 & Romaniello et al. (2008) & 0.47 & Soszynski et al. (2008); Soszyñski et al. (2010) \\
\hline HV 837 & 42.7563 & -0.82 & Romaniello et al. (2008) & 0.92 & Caldwell et al. $(1986,2001)$ \\
\hline HV 847 & 27.1019 & -0.76 & Romaniello et al. (2008) & 0.96 & Soszynski et al. (2008); Soszyñski et al. (2010) \\
\hline HV 865 & 33.3426 & -0.85 & Romaniello et al. (2008) & 1.14 & Caldwell et al. $(1986,2001)$ \\
\hline HV 1365 & 12.4165 & -0.83 & Romaniello et al. (2008) & 0.77 & Caldwell et al. $(1986,2001)$ \\
\hline HV 1954 & 16.7109 & -0.76 & Romaniello et al. (2008) & 0.79 & Caldwell et al. $(1986,2001)$ \\
\hline HV 2064 & 33.6512 & -0.64 & Romaniello et al. (2008) & 0.94 & Soszynski et al. (2008); Soszyñski et al. (2010) \\
\hline HV 2195 & 41.7830 & -0.68 & Romaniello et al. (2008) & 0.87 & Soszynski et al. (2008); Soszyñski et al. (2010) \\
\hline HV 2209 & 22.6464 & -0.66 & Romaniello et al. (2008) & 0.61 & Soszynski et al. (2008); Soszyñski et al. (2010) \\
\hline HV 11211 & 21.3796 & -0.82 & Romaniello et al. (2008) & 0.92 & Caldwell et al. $(1986,2001)$ \\
\hline HV 821 & 127.3140 & -0.79 & Luck et al. (1998) & 1.14 & Soszynski et al. (2008); Soszyñski et al. (2010) \\
\hline HV 824 & 65.8635 & -0.75 & Luck et al. (1998) & 0.99 & Soszynski et al. (2008); Soszyñski et al. (2010) \\
\hline HV 829 & 85.1990 & -0.65 & Luck et al. (1998) & 0.76 & Soszynski et al. (2008); Soszyñski et al. (2010) \\
\hline HV 834 & 73.6390 & -0.66 & Luck et al. (1998) & 0.47 & Soszynski et al. (2008); Soszyñski et al. (2010) \\
\hline HV 11157 & 69.0872 & -0.76 & Luck et al. (1998) & 0.43 & Soszynski et al. (2008); Soszyñski et al. (2010) \\
\hline \multicolumn{6}{|c|}{$\mathrm{LMC}$} \\
\hline G 458 & 74 & -0.4 & Luck \& Lambert (1992) & 0.48 & van Genderen/van Genderen \& Nitihardjo (1989); Caldwell et al. (2001) \\
\hline HV 1003 & 24.4 & -0.75 & Luck \& Lambert (1992) & 1.20 & Karczmarek et al. (2012) \\
\hline HV 1013 & 24.0991 & -0.60 & Romaniello et al. (2008) & 0.95 & Karczmarek et al. (2012) \\
\hline HV 1023 & 26.6073 & -0.28 & Romaniello et al. (2008) & 0.87 & Martin \& Warren (1979) \\
\hline HV 12452 & 8.7297 & -0.36 & Romaniello et al. (2008) & 0.82 & Soszynski et al. (2008); Soszyñski et al. (2010) \\
\hline HV 12700 & 8.1470 & -0.36 & Romaniello et al. (2008) & 0.56 & Martin \& Warren (1979) \\
\hline HV 2257 & 39.3699 & -0.36 & Luck et al. (1998) & 1.35 & Karczmarek et al. (2012) \\
\hline HV 2260 & 12.9420 & -0.37 & Romaniello et al. (2008) & 0.85 & Soszynski et al. (2008); Soszyñski et al. (2010) \\
\hline HV 2294 & 36.5595 & -0.42 & Romaniello et al. (2008) & 1.27 & Karczmarek et al. (2012) \\
\hline HV 2337 & 6.8707 & -0.36 & Romaniello et al. (2008) & 0.87 & Soszynski et al. (2008); Soszyñski et al. (2010) \\
\hline HV 2338 & 42.1944 & -0.37 & Luck et al. (1998) & 1.22 & Karczmarek et al. (2012) \\
\hline HV 2352 & 13.6144 & -0.48 & Romaniello et al. (2008) & 0.88 & Karczmarek et al. (2012) \\
\hline HV 2369 & 48.3059 & -0.63 & Romaniello et al. (2008) & 1.14 & Karczmarek et al. (2012) \\
\hline HV 2405 & 6.9183 & -0.28 & Romaniello et al. (2008) & 0.47 & Soszynski et al. (2008); Soszyñski et al. (2010) \\
\hline HV 2447 & 118.3115 & -0.26 & Luck et al. (1998) & 0.67 & Karczmarek et al. (2012) \\
\hline HV 2580 & 16.9044 & -0.25 & Romaniello et al. (2008) & 0.98 & Karczmarek et al. (2012) \\
\hline HV 2733 & 8.7297 & -0.28 & Romaniello et al. (2008) & 0.43 & Martin \& Warren (1979) \\
\hline HV 2793 & 19.1867 & -0.11 & Romaniello et al. (2008) & 0.84 & Soszynski et al. (2008); Soszyñski et al. (2010) \\
\hline HV 2827 & 78.8860 & -0.36 & Romaniello et al. (2008) & 0.63 & Karczmarek et al. (2012) \\
\hline HV 2836 & 17.5388 & -0.18 & Romaniello et al. (2008) & 1.06 & Martin \& Warren (1979) \\
\hline HV 2864 & 10.9901 & -0.20 & Romaniello et al. (2008) & 0.81 & Martin \& Warren (1979); Caldwell et al. (1986) \\
\hline HV 2883 & 108.9680 & -0.20 & Luck et al. (1998) & 1.24 & Martin \& Warren (1979); van Genderen (1983); Freedman et al. (1985) \\
\hline HV 5497 & 99.3116 & -0.25 & Romaniello et al. (2008) & 0.66 & Karczmarek et al. (2012) \\
\hline HV 6093 & 4.7863 & -0.6 & Romaniello et al. (2008) & 0.81 & Soszynski et al. (2008); Soszyñski et al. (2010) \\
\hline HV 877 & 45.0817 & -0.46 & Romaniello et al. (2008) & 0.92 & Karczmarek et al. (2012) \\
\hline HV 879 & 36.8129 & -0.14 & Romaniello et al. (2008) & 1.28 & Karczmarek et al. (2012) \\
\hline HV 883 & 133.5850 & -0.19 & Luck et al. (1998) & 1.41 & Karczmarek et al. (2012) \\
\hline HV 900 & 47.5085 & -0.43 & Luck et al. (1998) & 1.09 & Karczmarek et al. (2012) \\
\hline HV 909 & 37.5654 & -0.27 & Luck et al. (1998) & 1.09 & Karczmarek et al. (2012) \\
\hline HV 953 & 47.9 & -0.47 & Luck \& Lambert (1992) & 1.08 & Karczmarek et al. (2012) \\
\hline HV 971 & 9.2897 & -0.29 & Romaniello et al. (2008) & 1.42 & Martin \& Warren (1979) \\
\hline HV 997 & 13.1522 & -0.22 & Romaniello et al. (2008) & 0.91 & Soszynski et al. (2008); Soszyñski et al. (2010) \\
\hline
\end{tabular}

Notes. From left to right the columns give the source ID, the period, the iron abundance available in the literature and its reference, the visual amplitude, and its reference. 
Table 7. continued.

\begin{tabular}{|c|c|c|c|c|c|}
\hline ID & $\begin{array}{l}\text { Period } \\
\text { (days) }\end{array}$ & {$[\mathrm{Fe} / \mathrm{H}]$} & Ref. & $\begin{array}{r}A_{V} \\
\mathrm{mag}\end{array}$ & Ref. \\
\hline \multicolumn{6}{|c|}{ NGC 1850} \\
\hline 9 & 30.4 & -0.12 & Sebo \& Wood (1995) & 1.2 & Sebo \& Wood (1995) \\
\hline 17 & 18.66 & -0.12 & Sebo \& Wood (1995) & 0.8 & Sebo \& Wood (1995) \\
\hline 110 & 11.858 & -0.12 & Sebo \& Wood (1995) & 1.0 & Sebo \& Wood (1995) \\
\hline 58 & 8.558 & -0.12 & Sebo \& Wood (1995) & 1.0 & Sebo \& Wood (1995) \\
\hline 269 & 7.01 & -0.12 & Sebo \& Wood (1995) & 0.9 & Sebo \& Wood (1995) \\
\hline 341 & 3.593 & -0.12 & Sebo \& Wood (1995) & 0.8 & Sebo \& Wood (1995) \\
\hline \multicolumn{6}{|c|}{ NGC 1866} \\
\hline HV 12197 & 3.14381 & -0.43 & Mucciarelli et al. (2011) & 0.77 & Welch et al. (1991) \\
\hline HV 12198 & 3.52279 & -0.43 & Mucciarelli et al. (2011) & 0.65 & Welch et al. (1991) \\
\hline HV 12199 & 2.63918 & -0.43 & Mucciarelli et al. (2011) & 0.69 & Welch et al. (1991) \\
\hline HV 12200 & 2.7248 & -0.43 & Mucciarelli et al. (2011) & 0.82 & Welch et al. (1991) \\
\hline HV 12202 & 3.10112 & -0.43 & Mucciarelli et al. (2011) & 0.55 & Welch et al. (1991) \\
\hline HV 12203 & 2.95411 & -0.43 & Mucciarelli et al. (2011) & 0.65 & Welch et al. (1991) \\
\hline HV 12204 & 3.43876 & -0.43 & Mucciarelli et al. (2011) & 0.93 & Welch et al. (1991) \\
\hline V4 & 3.3157 & -0.43 & Mucciarelli et al. (2011) & 0.25 & Welch et al. (1991) \\
\hline V6 & 1.944252 & -0.43 & Mucciarelli et al. (2011) & 0.38 & Welch et al. (1991) \\
\hline V7 & 3.453 & -0.43 & Mucciarelli et al. (2011) & 0.46 & Welch et al. (1991) \\
\hline V8 & 2.0088 & -0.43 & Mucciarelli et al. (2011) & 0.31 & Welch et al. (1991) \\
\hline We 2 & 3.054847 & -0.43 & Mucciarelli et al. (2011) & 0.65 & Welch \& Stetson (1993) \\
\hline We 3 & 3.045 & -0.43 & Mucciarelli et al. (2011) & 0.60 & Welch \& Stetson (1993) \\
\hline We 4 & 2.8604 & -0.43 & Mucciarelli et al. (2011) & 0.45 & Welch \& Stetson (1993) \\
\hline We 6 & 3.289 & -0.43 & Mucciarelli et al. (2011) & 0.50 & Welch \& Stetson (1993) \\
\hline We 8 & 3.043 & -0.43 & Mucciarelli et al. (2011) & 0.51 & Welch et al. (1991) \\
\hline WS 11 & 3.0544 & -0.43 & Mucciarelli et al. (2011) & 0.40 & Welch \& Stetson (1993) \\
\hline WS 5 & 2.8950 & -0.43 & Mucciarelli et al. (2011) & 0.40 & Welch \& Stetson (1993) \\
\hline WS 9 & 3.0710 & -0.43 & Mucciarelli et al. (2011) & 0.44 & Welch et al. (1991) \\
\hline
\end{tabular}

